\title{
Outcomes for COPD pharmacological trials: from lung function to biomarkers
}

\begin{abstract}
M. Cazzola, W. MacNee, F.J. Martinez, K.F. Rabe, L.G. Franciosi, P.J. Barnes, V. Brusasco, P.S. Burge, P.M.A. Calverley, B.R. Celli, P.W. Jones, D.A. Mahler, B. Make, M. Miravitlles, C.P. Page, P. Palange, D. Parr, M. Pistolesi, S.I. Rennard, M.P. Rutten-van Mölken, R. Stockley, S.D. Sullivan, J.A. Wedzicha and E.F. Wouters on behalf of the American Thoracic Society/European Respiratory Society Task Force on outcomes of COPD
\end{abstract}

ABSTRACT: The American Thoracic Society/European Respiratory Society jointly created a Task Force on "Outcomes for COPD pharmacological trials: from lung function to biomarkers" to inform the chronic obstructive pulmonary disease research community about the possible use and limitations of current outcomes and markers when evaluating the impact of a pharmacological therapy. Based on their review of the published literature, the following document has been prepared with individual sections that address specific outcomes and markers, and a final section that summarises their recommendations.

\section{CONTENTS}

Abstract

\section{Background}

M. Cazzola, W. MacNee, F.J. Martinez, K.F. Rabe and L.G. Franciosi

\section{Lung function}

V. Brusasco and F.J. Martinez

Patient-reported outcomes

P.W. Jones and D.A. Mahler

Exacerbations

J.A. Wedzicha and M. Miravitlles

Exercise

P.M.A. Calverley and P. Palange

Mortality

B.R. Celli and P.S. Burge

Social and economic burden

S.D. Sullivan and M.P.M.H. Rutten-Van Mölken

Imaging

R. Stockley, M. Pistolesi and D. Parr

Nonpulmonary markers

E.F. Wouters and S.I. Rennard

Minimal important difference

B. Make

Biomarkers

P.J. Barnes and C. P. Page

Summary and conclusions

References

449 451

For editorial comments see page 238 .
AFFILIATIONS

For affiliations, please see the

Acknowledgements section.

CORRESPONDENCE

M. Cazzola

Unit of Respiratory Diseases

Dept of Internal Medicine

University of Rome "Tor Vergata"

Via Montpellier 1

00133 Rome

Italy

Fax: 390672596621

E-mail: mario.cazzola@uniroma2.it; mcazzola@qubisoft.it

Received:

July 312006

Accepted after revision:

November 202007

SUPPORT STATEMENT

This Task Force has received a grant for its activity directly from the ERS.

STATEMENT OF INTEREST Statements of interest for F.J. Martinez and S.I. Rennard can be found at www.erj.ersjournals.com/ misc/statements.shtml 


\section{BACKGROUND}

Clinical trials in chronic obstructive pulmonary disease (COPD) normally include forced expiratory volume in one second (FEV1), the principal measure of lung function, as an outcome, mainly because the COPD research community and regulatory agencies have traditionally recognised its importance as an objective index of airflow obstruction that measures both symptomatic relief and disease progression [1]. International bodies, such as the Global Initiative for Chronic Obstructive Lung Disease (GOLD), the American Thoracic Society (ATS) and the European Respiratory Society (ERS), have been working together to promote the use of FEV1 as a means of defining and staging this disease. With their extensive efforts, COPD is now known as a "disease state characterised by airflow limitation that is not fully reversible" [2].

However, many researchers still have difficulty defining what constitutes a response to a pharmacological intervention in COPD [3]. They are faced with a multicomponent disease characterised by a range of pathological changes, which include mucus hypersecretion, airway narrowing and loss of alveoli in the lungs, and loss of lean body mass and cardiovascular effects at a systemic level. COPD patients are also heterogeneous in terms of their clinical presentation, disease severity and rate of disease progression [4]. Their degree of airflow limitation, as measured by FEV1, is also known to be poorly correlated to the severity of their symptoms or health-related quality of life (HRQoL), which adds to the difficulties of researchers who are trying to improve the definition of COPD and current disease staging systems.

Since the relationship between spirometry and symptoms appears to be poor, measures of lung physiology alone may not adequately describe both the social impact of COPD and the effectiveness of therapeutic interventions in individual patients [4]. Most researchers regard changes in patientcentred outcomes, such as symptoms, exacerbations, exercise capacity and HRQoL, as important as or more important than changes in lung function. A panel of COPD experts have recently highlighted the importance of such outcomes and indicated the need for a more comprehensive assessment of both disease progression and treatment efficacy. Thus, they have proposed a multidimensional measurement for COPD that encompasses FEV1, the modified Medical Research Council (MRC) dyspnoea scale and the body mass index (BMI) [5]. This development reflects the need for better understanding regarding newly proposed and existing COPD measures so that researchers and regulatory agencies can make better informed decisions when assessing new drug therapies for COPD, but also shows the challenges of abandoning the traditional outcome measures, such as FEV1 and BMI, that possess no clearly defined relationship with patient-centred outcomes.

\section{Methods}

The ATS/ERS jointly created a Task Force on "Outcomes for COPD pharmacological trials: from lung function to biomarkers" to inform the COPD research community about the possible use and limitations of current outcomes and markers when evaluating the impact of a pharmacological therapy. Based on their review of the published literature, the following report has been prepared with individual sections that address specific outcomes and markers, and a summary of their recommendations.

\section{Task Force selection}

Task Force members were selected using the following criterion: worldwide recognised experts in COPD trials and in specific COPD outcomes. An initial list was prepared by those who developed the proposal and this list was integrated with other names suggested by the ERS Scientific Committee and by the three reviewers of the application, considering the expertise in COPD trials and in specific outcomes.

\section{Outcomes assessed}

The major sections of the present report are: Lung function; Patient-reported outcomes; Exacerbations; Exercise; Mortality; Social and economic burden; Imaging; Nonpulmonary markers; Minimal important difference; and Biomarkers. Each outcome measure was assessed by a group of authors using the set of criteria described in table 1 . This assessment was left to the discretion of the authors. Throughout this process, the authors have considered an "outcome" to mean a consequence of the disease that a patient normally experiences. This may be cough, dyspnoea, weight loss, exercise intolerance, exacerbations, impaired HRQoL, increased health resource use or mortality. Conversely, a "marker" is a measurement known to be associated with an outcome; for example, exercise capacity as tested in a laboratory is a marker of exercise intolerance in daily life [4].

\section{Literature review}

The authors searched the literature according to strategies that they developed independently. No central literature review or standardised evaluation for inclusion or exclusion of evidence was applied. Thus, while the ATS/ERS Task Force has adopted a comprehensive approach to reviewing the measures reported in the present report, it does acknowledge that not all measures and all evidence may have been included due to the amount of evidence available on each measure in the medical literature.

\section{LUNG FUNCTION \\ Introduction}

COPD is characterised by physiological abnormalities, including airflow limitation, abnormalities in gas exchange and lung hyperinflation. These can be objectively assessed in the laboratory using measurements such as FEV1, arterial oxygen tension $\left(\mathrm{Pa}_{\mathrm{a}} \mathrm{O}_{2}\right)$ and carbon dioxide arterial tension from blood gas determinations, as well as lung volumes measured at rest or during exercise. These markers serve as objective physiological measurements that aid in diagnosing disease, assessing its severity and analysing the mechanisms underlying some of its morbidity. In COPD, FEV1 is used in diagnosis and staging, arterial blood gases are useful in defining respiratory failure and dynamic hyperinflation helps to explain exertional dyspnoea [6].

Unfortunately, it is not easy to determine whether a measured change reflects a true change in pulmonary status or is only a result of test variability. Appropriate concepts such as sensitivity to change or responsiveness should be applied. These concepts account for test variability or variability in the 


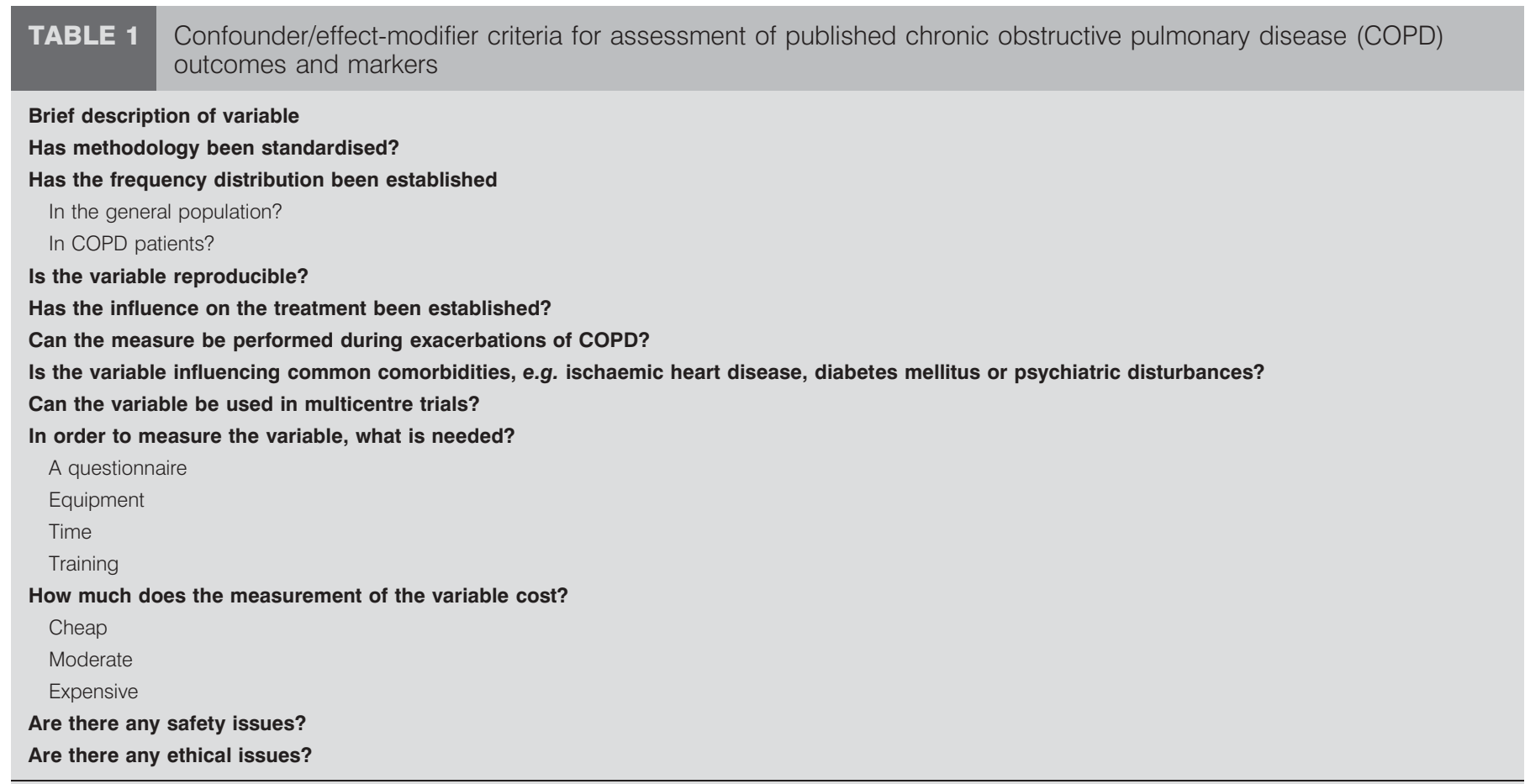

A confounder can be defined as a variable that can cause or prevent the outcome of interest, is not an intermediate variable and is associated with the factor under investigation. An effect modifier can be defined as a factor that modifies the effect of a putative causal factor under study.

measurement of differences at two different time-points. Repeated measures indicating change from baseline will provide more confidence that real change has occurred.

\section{Forced expiratory manoeuvre outcomes}

FEV1 is the volume of air that is forcibly exhaled in the first second, whereas forced vital capacity (FVC) is the total volume of air exhaled after a full inspiration. The methodology for obtaining forced expiratory manoeuvres and derived parameters have been standardised by the ATS and the ERS first separately and now also jointly [7]. In the general population, reference equations based on the distribution of spirometric parameters in normal populations have been made available by the ATS [8], by the European Community for Coal and Steel (ECCS) [9] and by studies on different ethnic groups and age ranges [10]. As such, it is important to carefully consider reference values that are most likely to represent the population to be tested in clinical practice or in multicentre clinical trials. In COPD patients, the FEV1 has been used to classify COPD patients by severity [11] and to describe progression [12] of the disease.

FEV1 and FVC have been shown to be highly reproducible in the vast majority of patients, with differences between highest and second highest values within $150 \mathrm{~mL}$ or $6 \%$, if obtained by well-trained technicians. Variability coefficients for FEV1 and FVC over time (within days, weeks or years) have been reported [10]. When taking into consideration the degree of change in FEV1 that would be considered clinically meaningful by the regulators, a change of $5-10 \%$ from baseline values is considered to be clinically important. During advisory committee meetings of the US Food and Drug Administration for new COPD drugs, a change of $<3 \%$ of the baseline has been deemed to be not clinically important [13]. According to ATS/ ERS recommendations for the interpretation of lung function tests [10], the change in FEV1 should be $\geqslant 20 \%$ in short-term trials (of weeks of duration) and $\geqslant 15 \%$ in long-term trials $(\geqslant 1 \mathrm{yr})$ to be confident that a clinically meaningful change has occurred (a summary of these recommendations can be found in the Minimal important difference section).

Forced expiratory volume in six seconds (FEV6) has recently been suggested as an alternative to FVC. It has been found to be $\sim 25 \%$ less variable than FVC [14]. The operating characteristics of this measurement continue to be defined according to reference values recently published $[15,16]$. The diagnostic accuracy for defining airflow obstruction or restriction seems good [17, 18], although this depends on the population tested and the use of appropriate lower limit thresholds to define normality [19]. It has been suggested that different fixed thresholds (FEV1/FEV6 $<73 \%$ and FEV $6<82 \%$ ) [20] are more appropriate than the currently utilised thresholds for obstruction in international guidelines (FEV1/FVC $<70 \%$ and FVC $<80 \%$ predicted).

In terms of health outcomes for the general population, a low pre-bronchodilator FEV1 has been found to be a predictor of mortality for all causes and cardiovascular mortality [21-25]. In COPD patients, post-bronchodilator FEV1 is poorly correlated with patient-centred outcomes, such as dyspnoea, exercise performance and HRQoL at baseline or after pharmacological interventions [26-31]. However, FEV1 has the advantage of being the most repeatable lung function parameter and one that measures changes in both obstructive and restrictive types of lung disease [7]. Therefore, FEV1 has been proposed as a 
measure to be used for adopting treatment strategies in COPD based on severity classification [15].

The European Medicines Agency [1] specifies that strict airways reversibility criteria must be applied to patient inclusion in clinical trials (e.g. $\leqslant 12-15 \% \mathrm{FEV} 1$ reversibility post-bronchodilator), with a pre-bronchodilator FEV1/FVC ratio $\leqslant 70 \%$ and FEV1 within $30-70 \%$ pred (post-bronchodilator). The ATS/ERS recommendation is that an increase in FEV1 and/or FVC $>12 \%$ and $200 \mathrm{~mL}$ compared with baseline during a single testing session suggests a significant bronchodilatation [10]. However, although changes in FEV1 and FVC have been suggested to assess the reversibility of airway obstruction, a negative response to a single administration of bronchodilator should not be used to determine the need for long-term bronchodilator treatment due to the lack of sensitivity of the parameters derived from full forced expiratory manoeuvres [32] and the variability of response over time [33]. FEV1, FVC and FEV1/FVC ratio have all been used as predictors of the outcome of lung volume reduction surgery [34].

During exacerbations of COPD, measurement of FEV1 is possible but it does not seem to be useful for the early detection of exacerbations [35]. However, even small changes in postbronchodilator FEV1 following acute treatment were found to be associated with meaningful clinical outcomes [36-38].

FEV1 has been used as a primary variable in the vast majority of multicentre trials, though an increase in FVC is also considered as proof of bronchodilatation [10], since physiologically relevant changes occur in a number of COPD patients who respond to bronchodilators with an increase of FVC without changes in FEV1 $[9,39]$. Reliable values of FVC may be difficult to obtain in severe COPD patients who are unable to sustain prolonged expiration for a period $>6 \mathrm{~s}$; therefore, FEV6 may be used instead of FVC. The rate of decline of FEV1 has been shown to be decreased by smoking cessation [12].

With respect to feasibility, spirometry can be undertaken using different types of equipment, provided the standard requirements are met [7]. Simple spirometry is only moderately timeconsuming, with an average of 10-20 min per session [40]. The reproducibility of spirometric measurements is critically dependent on the ability of the technician [7]. Thus, specific training is mandatory for the technical staff and also for physicians who wish to undertake spirometry testing [36]. The cost of spirometers is widely variable, but relatively inexpensive portable equipment can be used for large clinical trials, provided accuracy requirements are met [7].

When spirometry is performed, patients can experience some discomfort, light-headedness or even syncope. Airway obstruction may occur if multiple prolonged exhalations are required for the measurement of FVC. The use of FEV6 may prevent these events in more severe patients. There is also potential for infection transmission during pulmonary function testing but direct evidence has not been provided [41]. Measures to reduce the risk of infection have been identified and should be followed [41].

\section{Lung volumes}

Functional residual capacity (FRC) is the volume of gas remaining in the lung at the end of tidal expiration. In healthy subjects at rest, the FRC corresponds to the relaxation volume
$(V \mathrm{r})$ of the respiratory system. In COPD, FRC may be increased because the $V \mathrm{r}$ is increased (static hyperinflation) or the endtidal lung volume exceeds $V_{\mathrm{r}}$ (dynamic hyperinflation). Inspiratory capacity (IC) is the maximum volume of gas that can be inspired from end-tidal expiration, which is the difference between total lung capacity (TLC) and FRC. Residual volume (RV) is the volume of gas remaining in the lung at the end of a maximal expiration. In COPD, RV may be increased due to airway closure or extreme flow limitation.

The methods for measuring absolute lung volume and its subdivisions have been standardised in a joint ATS/ERS document $[7,42]$. In the general population, reference equations based on the distribution of FRC and RV in normal populations have been published by the ECCS [9]. Published reference values are summarised in the ATS/ERS joint document on interpretation of pulmonary function tests [10]. Differences in ethnicity are not well characterised. Most laboratories use reference values recommended by ECCS [9] or an ATS/ERS workshop [43]. There are no equations for predicting IC; it is thus determined by the subtraction of predicted TLC from predicted FRC. Currently, there are no frequency distribution data available for any of these spirometry measures in COPD patients.

The reproducibility of FRC has been reported, with coefficients of variation ranging 3.5-6.7\% for plethysmography and 4.9$10.4 \%$ for helium dilution, without apparent differences between normal and obstructed subjects [44]. The coefficients of variation of RV measurements ranged 9.5-12.4\% for plethysmography and $2.4-14 \%$ for helium dilution. However, no data are available for FRC or RV reproducibility in absolute values. Inter-laboratory differences seem to be as important as intra-individual differences. For IC, the short-term variability (95\% confidence interval (CI)) in subjects with chronic airway obstruction at rest was $220-150 \mathrm{~mL}$ or $10-4.5 \%$ pred $[32,45]$.

In COPD patients, FRC increments [46] or IC decrements [29, $45,47-54]$ have been shown to correlate better than increased FEV1 with exercise tolerance and dyspnoea, and HRQoL, both at baseline $[45,47,51,52]$ and after pharmacological $[29,46,49$, $50,53]$ or surgical $[48,54]$ interventions. When related to TLC, IC has been reported to be an independent predictor of mortality in COPD of different severity [55]. Changes in RV have been shown to be a major determinant of response to lung volume reduction surgery [56], but its usefulness in large studies has yet to be determined.

Changes in lung volumes can occur in COPD patients after treatment with bronchodilators, even in the absence of changes in FEV1 [32, 57]. Small increases in IC after bronchodilator therapy, which signify a reduction in end-expiratory lung volume, are associated with reduced mechanical loading and increased functional strength of the inspiratory muscles. This in turn results in a decreased work of breathing and a reduced oxygen debt. Furthermore, an increased resting IC (of the order of $0.3 \mathrm{~L}$ or $10 \%$ pred) would be indicative of a greater ability to expand tidal volume during exercise, with a resultant increase in ventilatory capacity $[29,49,58]$. Reduced operating volumes during exercise enhance the neuromechanical coupling of the respiratory system (i.e. the relationship between neural drive and mechanical response), thereby relieving respiratory 
discomfort. The net effect of these physiological benefits is an improvement in the patient's ability to engage in exercise [59]. Several studies have also indicated that long-acting bronchodilators can reduce hyperinflation in COPD, as measured by RV, FRC and IC, in a manner that is somewhat similar to that seen with lung volume reduction surgery $[49,60,61]$.

During recovery from mild exacerbations, both FRC and RV were found to decrease, while IC increased and TLC remained constant [38]. Therefore, IC can be used to reflect changes in lung hyperinflation during acute exacerbations. Studies have confirmed that TLC does not change during more severe exacerbations $[38,62]$.

These variables can be measured in multicentre trials, possibly in selected centres. For acute interventions, in which TLC can be assumed to be constant [63-65], changes in IC are a good surrogate for changes in FRC. For chronic interventions, in which TLC may not be constant, changes in IC may not mirror changes in FRC [60].

In terms of feasibility, FRC and RV measurements require a body plethysmograph or spirometers with inert gas analysers. Either method can be used, provided the equipment meets the standard requirements [42]. However, they are not interchangeable, since moderate-to-severe airflow obstruction dilution methods tend to underestimate and body plethysmography tends to overestimate TLC. IC can be measured during simple spirometry if a closed circuit system is used. Dilution methods are too time-consuming for serial measurements, whereas body plethysmography is much less time-consuming and does not add much time to that necessary for simple spirometry. IC is part of the spirometric manoeuvre and only requires a few stable breaths before its measurement. The reproducibility of pulmonary function test measurements is critically dependent on the ability of the technician [41]. Measurement of absolute lung volumes is technically more demanding than simple spirometry and specific training is necessary. The equipment for measuring lung volumes is substantially more expensive than simple spirometers. Body plethysmographs are more expensive than dilution equipment but the extra cost may be neutralised by time saving.

\section{Gas exchange}

The diffusing capacity of the lung for carbon monoxide (DL,CO) is a measure of carbon monoxide (CO) transfer from the airspace to pulmonary capillary blood; it is expressed as the total uptake of $\mathrm{CO}$ by the lung per unit of time and per unit of driving pressure $[66,67]$.

The methodology of the single-breath method has been standardised by the ATS and the ERS first separately [66, 67] and then jointly [68]. For the general population, reference equations based on the distribution of $D \mathrm{~L}, \mathrm{CO}$ values in normal populations have been made available by studies on different ethnic groups and age ranges [69-71]. DL,CO has been used to quantify the extent of emphysema in COPD patients [72]. Measurements have been made before and after rehabilitation [73] and have been used to assess patients for lung volume reduction surgery [74, 75].

The measure of $D \mathrm{~L}, \mathrm{CO}$ is reproducible even if the interlaboratory coefficient of variation is larger than for spirometry measurements. Acceptable DL,CO test criteria have been standardised [68]. A coefficient of variation of 3-4\% has been reported in repeated measurements in normal subjects and in patients with abnormal spirometric patterns. An inter-sessional $D$ L,CO variability of $\leqslant 9 \%$ over time has been reported [76-79].

The influence of $\mathrm{DL}, \mathrm{CO}$ on health outcome has not been established in the general population, whereas in COPD patients it has been used in the evaluation of surgical risk, particularly for lung cancer and other thoracic surgery [80]. $D \mathrm{~L}, \mathrm{CO}$ has also been used in studies evaluating the effects of lung volume reduction surgery [74, 75], rehabilitation [73] and therapy for $\alpha_{1}$-antitrypsin deficiency [81, 82]. However, it is a measure that can only be performed in stable conditions rather than during exacerbations of COPD.

DL,CO has been used as a primary outcome variable in multicentre trials, particularly in studies of lung volume reduction surgery $[75,76]$. It can be measured using different types of equipment, provided they meet the standard requirements [66, 67]. The measurement takes 15-30 min and its reproducibility is critically dependent on the ability of the technician and on the achievement of acceptable test criteria $[66,67]$. Therefore, specific training is mandatory for the technical staff $[66,67]$. The cost of $D L, C O$ equipment is variable but rather expensive. It is not available in primary care although it can be used for limited clinical trials, provided accuracy requirements are met $[66,67]$. Discomfort, lightheadedness or even syncope may occur if multiple prolonged exhalations are required for measurement of $\mathrm{DL}, \mathrm{CO}$. There is also the possibility of infection transmission, although direct evidence has not been provided. General considerations for pulmonary function testing also apply to DL,CO equipment and procedures [41].

$\mathrm{Pa}_{1} \mathrm{O}_{2}$ is a measurement of arterial oxygen partial pressure and it can be determined by direct blood sampling. Some investigators have utilised arterialised earlobe capillary blood to assess $\mathrm{Pa}_{2} \mathrm{O}_{2}$, although potential for underestimation of arterial oxygenation has been suggested by several groups [83, 84]. Conversely, arterial oxygen saturation $\left(\mathrm{Sp}, \mathrm{O}_{2}\right)$ can be measured directly [85-87] or indirectly with the aid of pulse oximetry [88, 89].

Neither the ATS nor the ERS have standardised the methodology for both these variables. However, there are many studies in the literature that consider the methodology, techniques and equipment required to measure arterial blood gases [85, 90, 91]. The frequency distribution of blood gas values has been established for the general population; reference equations based on the distribution of $\mathrm{Pa}_{\mathrm{a}} \mathrm{O}_{2}$ in normal populations have been made available by studies on different ethnic groups and age ranges $[92,93]$. In COPD patients, $\mathrm{Pa}_{3} \mathrm{O}_{2}$ has been used to define respiratory failure $\left(\mathrm{Pa}_{\mathrm{a}_{2}}<7.98 \mathrm{kPa}(<60 \mathrm{mmHg})\right)$ and it was included for adopting treatment strategies in COPD based on severity classification and disease progression [11]. In terms of measurement reproducibility, modern blood gas analysers are automated self-diagnostic instruments that require minimal maintenance. Calibration of the machine's electrodes is important to correct for any drift in the measurements over time [85-87]. A coefficient of variation of $3-4 \%$ has been reported for many available pulse oximeters [90, 91]. 
In terms of health outcomes, $\mathrm{Pa}_{1} \mathrm{O}_{2}$ is useful for predicting survival status in hospitalised COPD patients with exacerbations [94, 95]. Conversely, $\mathrm{Sp}, \mathrm{O}_{2}$ is commonly used in sleep studies $[96,97] . \mathrm{Pa}_{2} \mathrm{O}_{2}$ has been used in many studies evaluating the effects of treatment in severe COPD patients. Since pulse oximetry is a noninvasive technique, $\mathrm{Sp}, \mathrm{O}_{2}$ can be easily determined in large studies that occur in primary care settings [98-102]. As mentioned previously, $\mathrm{Pa}_{2} \mathrm{O}_{2}$ is an important variable that can be measured in severe, uncooperative COPD patients with exacerbations [103]. $\mathrm{Sp}, \mathrm{O}_{2}$ can also be easily used to monitor these patients $[104,105]$. Both variables can be obtained in all patients with or without comorbidities.

$\mathrm{Pa}_{\mathrm{a}} \mathrm{O}_{2}$ and $\mathrm{Sp}_{\mathrm{p}} \mathrm{O}_{2}$ can be useful in multicentre trials in severe COPD patients, provided the same equipment is used in each setting and standard requirements are met [85, 91]. Both variables take little time to measure and the only training that is required is related to arterial blood sampling [86, 87]. The cost of the equipment for the measurement of $\mathrm{Pa}_{1} \mathrm{O}_{2}$ and arterial oxygen saturation is variable but rather expensive. $\mathrm{Sp}_{\mathrm{p}} \mathrm{O}_{2}$ that is obtained by pulse oximetry is relatively inexpensive and is available in almost all patient care settings. There are safety issues with arterial puncture for blood gas analysis, such as local pain, discomfort, light-headedness, nerve damage or syncope. Although the risk of bleeding may be increased, this procedure has been successfully performed in individuals who have been treated with therapeutic anticoagulation [106].

\section{Safety and technical considerations}

In general, pulmonary function testing can be physically demanding for a minority of patients when considering the influence on common comorbidities, e.g. ischaemic heart disease, diabetes mellitus or psychiatric disturbances. It is recommended that patients are not tested within 1 month of myocardial infarction [42]. Conversely, measurements may be suboptimal in the presence of various comorbidities, such as chest or abdominal pain, facial abnormalities, stress incontinence or psychiatric disturbances. With respect to safety issues, claustrophobia may occur during body plethysmography. The potential also exists for infection transmission but direct evidence has not been provided [41].

Measurements of absolute lung volumes may also be suboptimal in the presence of various comorbidities, such as chest or abdominal pain, facial abnormalities, stress incontinence or psychiatric disturbances.

\section{Conclusions}

Based on the review of lung function measurements, FEV1 remains a primary end-point that regulatory authorities regard as an acceptable measure of efficacy for COPD pharmacological trials, particularly in combination with instruments that encompass symptomatic-based end-points [1]. However, since lung hyperinflation and its reduction in response to a bronchodilator are not reflected in routine spirometry, IC could also be included in COPD trials, particularly where changes in lung physiology are expected. In hyperinflated patients, RV or FVC are useful measures for the identification of a therapeutic response that may not be determined from measuring FEV1. It has also been highlighted that measuring IC after a pharmacological intervention without plethysmographic determination of the static lung volumes may not be an adequate reflection of the underlying changes in these volumes [53]. Arterial blood gases are useful outcomes in interventional studies that might affect respiratory drive or impair ventilation-perfusion relationships in the lungs.

\section{PATIENT-REPORTED OUTCOMES}

\section{Introduction}

The development of COPD may affect several aspects of a patient's health [107]. These consequences of illness can be regarded as a process of illness progression, which normally starts at the development of physiological or biological abnormalities, resulting in symptoms and physical limitations that are noticed and reported by the patients. Eventually, patients will have to face their inability to take part in their usual activities, which will influence their perception of their health and ultimately their general well-being [108].

As a consequence, a number of clinical and physiological outcomes, such as dyspnoea, functional status, HRQoL and health status, are recognised as being important for the characterisation of response to treatment [109]. For instance, dyspnoea is the primary reason for patients seeking medical care. Measurements of dyspnoea provide an insight into the practical effects of treatment on everyday life, reflecting whether or not patients perceive an improvement in this primary symptom of COPD. Patients with COPD frequently decrease their activity in order to avoid the unpleasant sensation of breathlessness. Functional status measurement reveals the number of activities that a patient can perform, something not reflected in measurements of FEV1 or dyspnoea.

\section{Health status and HRQoL}

Health status measurement provides a standardised method of assessing the impact of disease on patients' daily lives, activity and well-being. The term "quality of life" is often used loosely in this context, but this is inappropriate. The factors that determine an individual's quality of life are varied. Even in very ill people, health usually forms only a minor determinant of an individual's quality of life, with employment, finances, family and social factors being collectively more important [110]. "HRQoL" is a more specific term.

Health status and HRQoL measurements are designed to allow comparisons across patients and studies. This means that all patients in all studies must be measured in the same way using a common instrument and unit of measurement. The measurements must be made without bias, i.e. the instrument should apply to relevant patients equally. Essentially, health status and HRQoL are instruments for use in groups of patients; they may also be used to assess individuals, but it should be understood that they will provide a standardised assessment of their health, not their quality of life.

The reader is also directed to the ATS Quality of Life website [111] as a useful source of information and to access detail of some of the questionnaires summarised herein.

\section{Types of health status and HRQoL instruments}

Generic health status

These questionnaires are designed to assess health irrespective of disease. A number have been used in COPD, in particular, 
the 36-item Short-Form Health Survey (SF-36), the Sickness Impact Profile (SIP) and the Nottingham Health Profile (NHP).

SF-36 was developed as a measure of general health [112]. It has eight domains: physical function; mental health; energy/ vitality; health perception; physical role limitation; mental role limitation; social function; and pain. Two summary scores may be calculated: physical summary and mental summary. It is self-completed and can be carried out online.

SIP was developed as a measure of general health [113]. It has 12 categories: sleep and rest; diet; work; home management; recreation and pastimes; ambulation; mobility; body care and movement; social interaction; alertness behaviour; emotional behaviour; and communication. Two sub-scores are produced: i.e. physical and psychosocial. It is self-completed.

NHP was developed as a measure of general health [114]. It has six domains: pain; physical mobility; emotional reactions; energy; social isolation; and sleep. It is also self-completed.

\section{Long disease-specific health status and HRQoL}

These questionnaires have been developed and validated in COPD patients. They are comprehensive, covering a range of aspects of COPD and are therefore reasonably time-consuming to complete. Examples include: the individualised Chronic Respiratory Questionnaire (CRQ); the St George's Respiratory Questionnaire (SGRQ); and the Quality of Life for Respiratory Illness Questionnaire (QoL-RIQ).

CRQ was developed in 1987 to measure HRQoL in patients with COPD [115]. It includes 20 questions across four domains: dyspnoea; emotional function; fatigue; and mastery. A selfadministered standardised (SAS) version, CRQ-SAS (which takes $7 \mathrm{~min}$ ), is available and has been validated against the original interviewer-administered version and for telephone administration [116-118].

SGRQ was developed in 1992 to measure health status in patients with respiratory disease [119]. It has also been validated for use in bronchiectasis [120]. It has three domains: symptoms; activity; and impacts. A total score is also calculated. It was designed for supervised self-administration.

QoL-RIQ was developed in 1997 for use in mild-to-moderate asthma and COPD [121]. It has seven domains: breathing problems; physical problems; emotions; situations triggering or enhancing breathing problems; general activities; daily and domestic activities; and social activities, relationships and sexuality. A total score is also calculated. It is self-administered and a shorter version has been described [122].

\section{Short disease-specific health status and HRQoL}

These questionnaires were developed to provide valid but shorter estimates of overall healthy status in COPD. They are less comprehensive than the long instruments. Examples of these questionnaires are: the CRQ-SAS; the Airways Questionnaire (AQ)20; and the Breathing Problems Questionnaire (BPQ). AQ20 was developed in 1998 for use in asthma [123] and COPD [124, 125]. BPQ was developed in 1994 for use in COPD [126], and was shortened in 1998 [127]. Both are self-administered questionnaires with a total score calculated.
Disease-specific health status and HRQoL for patients with respiratory failure

There is only one questionnaire in this class that assesses patients with severe hypoxia or ventilatory failure. The Maugeri Foundation Respiratory Failure questionnaire was developed in 1999 for use in patients with respiratory failure [128]. It has three domains: daily activity; cognitive function; and invalidity. A total score is calculated and is also selfadministered.

\section{COPD control questionnaires}

The Clinical Control Questionnaire is the only questionnaire that exists in this class. It was developed in 2003 to assess the quality of control of COPD from the physician's perspective [129]. It has three domains: symptom; functional state; and mental state. A total score is calculated and it can be selfadministered.

\section{Functional status questionnaire}

These questionnaires are the same length as the long diseasespecific health status questionnaires but address functional status (which is more than just mobility) in more detail than those instruments. The two most common questionnaires are Pulmonary Functional Status and Dyspnoea Questionnaire (PFSDQ-M) and Pulmonary Functional Status Scale (PFSS).

PFSDQ-M and PFSS were both developed in 1998 for use in patients with COPD [130, 131]. PFSDQ-M has two domains, activity level and dyspnoea, whereas PFSS has three subdomains, daily activities/social functioning, psychological functioning and sexual functioning. Both are self-administered questionnaires with a total score calculated.

\section{Activity of daily living scales}

The Nottingham Extended Activity of Daily Living (EADL) scale and the London Chest Activity of Daily Living (LCADL) scale are the principal questionnaires in this class and have been designed for patients with more severe disease, largely more housebound than usual COPD patients.

The Nottingham EADL scale was developed in 1987 as a general measure of limitation of essential activities of daily living for stroke patients [132] but has been used in COPD [133]. It contains four domains: mobility; domestic; kitchen; and leisure. A total score is calculated and a clinician administers it.

The LCADL scale was developed in 2000 as a measure of activities of daily living in patients with severe COPD [134]. A total score is calculated and it is self-administered.

\section{Preference-based instruments}

These are generic scales from health to death designed to rank patients' health or preference for a health state. Complex direct methods of assessing health can be used, such as Standard Gamble or Time Trade-off techniques, or simpler methods, such as the feeling thermometer, a simple visual analogue scale (VAS), can be used to estimate an individual's preference score [135-138].

Indirect preference instruments provide ratings that are based on translation of scores through reference equations from other outcome measures. In that respect, they are unlike any other 
scale discussed herein. One of these, the Short-Form 6 Dimensions, an indirect utility instrument, is derived from an existing generic instrument, the SF-36. Other common questionnaires in this class of scales are the Quality of Wellbeing scale (QWB) and the European Quality of Life Questionnaire (EQ-5D). They are, even more than the other health status questionnaires, very much population-based.

QWB was developed as a utility instrument [139]. It addresses symptoms, mobility, physical activity and social activity. Scores can be translated into economic evaluation for costeffectiveness studies or quality-adjusted life-years (QALYs). It is administered by the interviewer.

EQ-5D was developed as a utility questionnaire [140]. It addresses mobility, self-care, usual activities, pain/discomfort and anxiety/depression. Scores can be translated into economic evaluation for cost-effectiveness studies or QALYs. It contains a feeling thermometer and is self-administered.

\section{General comments on available questionnaires in COPD}

There is a wide range of questionnaires available. However, certain generalisations can be made.

Disease-specific instruments tend to be more sensitive to change (more responsive) and therefore better suited than generic instruments to measure treatment effects in COPD. Of the three generic instruments listed herein, SF-36 is the most widely used and is well supported by a comprehensive website. Of the disease-specific measures, CRQ and SGRQ have been used very widely and there is extensive literature concerning them both. They have slightly different properties. Both have been used in rehabilitation and pharmaceutical studies: CRQ more in rehabilitation and SGRQ in long-term pharmaceutical studies particularly. Both are supported by the groups that developed them and are subject to continuing developmental work. The published literature concerning QoL-RIQ is much more limited.

The comprehensive functional status questionnaires are well established and are similar to the comprehensive diseasespecific questionnaires in many respects, but were developed from a narrower perspective. All of the remaining questionnaires listed have been developed to address perceived weaknesses in the long disease-specific instruments. They all have evidence for validity in their chosen applications and patient populations, but there is limited evidence as to whether they have the same level of validity, reliability and responsiveness as the long health status questionnaires when compared side by side in the same patients. It is also unclear whether they contribute any additional information.

The preference instruments are of greatest interest to health economists since they can be scaled from death to health, which means that data from patients who have died can be included in health economic analyses. QWB and EQ-5D have been used quite widely in COPD but, as with all generic instruments, they are relatively insensitive. The feeling thermometer has shown relatively good responsiveness [135-138].

\section{Details of questionnaires}

Details of some of the properties of these questionnaires are provided hereafter. No attempt has been made to list all the references that provide validation data for each instrument, although the references listed provide important validation data on each questionnaire. All have been validated to a varying degree. The most extensively used questionnaires are CRQ and SGRQ, and a PubMed search will provide a very wide range of references on these instruments. For many of the instruments listed herein, few additional references are available. Websites are available for a number of these instruments (particularly for the generic and utility instruments).

\section{Dyspnoea}

Dyspnoea, or breathlessness, can be measured based on the principles of psychophysics (stimulus-response relationship). In general, two different approaches have been used to measure dyspnoea in clinical trials: clinical ratings based on activities of daily living and ratings during an exercise task.

The purposes of measuring dyspnoea in pharmacological trials include differentiating between individuals who have less dyspnoea and those who have more (a discriminative instrument), and determining how much dyspnoea has changed (an evaluative instrument). For the assessment of treatment efficacy, the two important measurement criteria of an evaluative instrument are responsiveness and construct validity [141]. Responsiveness refers to the ability to detect change; if a treatment results in an improvement in dyspnoea, both the investigator and the clinician want to be confident that they can detect the difference, even if it is small. For construct validity, changes in dyspnoea scores should correlate with expected changes in other variables, such as lung function and exercise performance, consistent with theoretically derived predictions. However, the magnitude of the correlations is typically modest, indicating that dyspnoea ratings and measures of lung function represent different constructs.

\section{Clinical ratings}

Over the past 50 yrs a variety of questionnaires or scales has been developed to quantify dyspnoea [141]. Many of the original instruments are considered one dimensional, which relate the severity of dyspnoea to various physical tasks (e.g. walking on a level surface or climbing stairs). More recently developed instruments have been multidimensional, which include additional factors that influence dyspnoea. The following sections will consider the three clinical scales most widely used to quantify dyspnoea. These include: the MRC scale [142] the Baseline and Transition Dyspnoea Indices (BDI and TDI, respectively) [143]; and the dyspnoea domain of CRQ [115].

\section{MRC scale}

The MRC scale is a five-point scale published in 1959 that considers certain activities, such as walking or climbing stairs, which provoke breathlessness [142]. In $<1 \mathrm{~min}$, the patient selects a grade on the MRC scale that most closely matches his/her severity of dyspnoea. The MRC scale is considered a discriminative instrument that can categorise patients with COPD in terms of their disability [144]. In one study of COPD patients [145], the frequency distribution of MRC values showed a tendency for lower ratings (i.e. less dyspnoea) in patients with COPD, whereas dyspnoea ratings with other instruments demonstrated a more normal distribution. In the short (2 days-2 weeks) and long term, MRC values are 
reproducible. In terms of their influence on the health outcome in COPD patients, factor analyses have demonstrated that dyspnoea scores are separate and distinct from lung function and exercise capacity [145-147]. However, the MRC scale is not satisfactory as an evaluative instrument to measure changes in dyspnoea, its broad grades are generally unresponsive to interventions such as pharmacotherapy [141]. It is also not a useful measure during exacerbations of COPD. It is possible that cardiac disease could influence the severity of dyspnoea. However, in randomised controlled trials (RCTs) involving patients with COPD, those patients with clinically significant cardiac disease are typically excluded from entry. With respect to the utility of the MRC scale in multicentre trials, it is not recommended since its broad grades are considered unresponsive to change.

\section{$B D /$ and TDI}

BDI and TDI were developed in 1984 so that a physician, nurse or technician could interview a patient in order to obtain a comprehensive understanding of the patient's severity of breathlessness based on three components: functional impairment; magnitude of task; and magnitude of effort [143]. BDI is a discriminative instrument used to quantify the severity of dyspnoea at an initial or baseline state, whereas TDI is an evaluative instrument used to quantify the changes in dyspnoea from the initial or baseline state.

The methodology of BDI and TDI has been standardised. With the original BDI/TDI, an interviewer would ask the patient various questions about how activities of daily living influenced the patient's breathlessness. According to the patient's responses to the BDI, the interviewer would select a grade, based on standard and specific criteria, for each of three components: 1) functional impairment, with grades $0-4 ; 2$ ) magnitude of task, with grades 0-4; and 3) magnitude of effort, with grades $0-4$. These components add up to a baseline total score ranging $0-12$; the lower the score, the more severe the dyspnoea. For grading changes in dyspnoea with TDI, the interviewer would ask the patient various questions about how activities of daily living influence changes in dyspnoea experienced by the patient compared with the initial or baseline state. The components and grades are as follows: 1) functional impairment, with grades $-3-3$; 2) magnitude of task, with grades -3-3; and 3) magnitude of effort, with grades $-3-3$. These add up to a transition total score ranging -9-9; the negative value indicates deterioration, whereas a positive value indicates improvement.

In 2004, the self-administered and computerised (SAC) versions of BDI and TDI were developed to provide more standardised criteria for measuring breathlessness [148]. For BDI, one criterion in the magnitude-of-task component changed from "climbing three flights of stairs" to "climbing one flight of stairs". In the case of TDI, there were two changes. To remind the patient of his/her original dyspnoea status, an insert was provided on the computer screen of the descriptor selected for the corresponding component of the BDI. A bidirectional VAS was also created for each component of the TDI. The patient presses an up or down key on the computer keyboard to move a vertical bar up for improvement or down for deterioration in dyspnoea compared with the baseline dyspnoea status.
In terms of the statistical frequency distributions for BDI and TDI, their ranges have been determined to be normally distributed in COPD patients [145, 149, 150]. They are both reproducible measures in the short (i.e. 2 days -2 weeks) and the long term.

With respect to their influence on health outcomes, factor analyses have demonstrated that dyspnoea scores are separate and distinct from lung function, exercise capacity and other outcomes in COPD patients [145-147]. Longitudinal studies have also demonstrated an expected decrement in the TDI score (i.e. more breathlessness) in patients with COPD over 12 yrs [151, 152]. Numerous RCTs have also demonstrated improvements in TDI with pharmacotherapy compared with placebo in patients with COPD [150-160]. In an observational study [36], BDI/TDI were shown to be valid and responsive measures of acute changes in dyspnoea associated with a COPD exacerbation. In an RCT [161], COPD patients had significant improvements in dyspnoea $(p=0.04)$ after 10 days of treatment for an exacerbation with prednisone plus standard therapy ( +4.0 units in TDI) compared with placebo plus standard therapy $(+2.1$ units in TDI).

In terms of feasibility, BDI/TDI have been used to measure dyspnoea with pharmacotherapy compared with placebo in numerous RCTs performed at multiple centres [152-160]. Both instruments require an interviewer and the questionnaire. For the SAC versions, a computer and the software program are required. It takes $\sim 3-4$ min for either the original or the SAC versions. For the original BDI/TDI instruments, the interviewer should have a basic knowledge of respiratory disease and view a training video or observe an interview between a patient and an experienced interviewer. When implementing the SAC versions, the patient needs to complete a practice session by rating "tiredness" on the computer before selecting a grade for each of three components of BDI or TDI. Interviewer-administered BDI and TDI are free to academic institutions and to individuals using the instruments in a research protocol. Both instruments can be obtained from the Mapi Research Institute in Lyon, France. When pharmaceutical companies use them in RCTs, a modest fee is charged per study and per language. The SAC versions of BDI and TDI are available from Psychological Applications in Waterbury, VT, USA. A fee is determined based on the intended use of the instruments.

\section{Dyspnoea component of CRQ}

CRQ was developed in 1987 to measure HRQoL in patients with respiratory disease [115]. Dyspnoea was one of four dimensions included in the CRQ. While the original dyspnoea domain was individualised (patients chose activities that made them most short of breath), the authors validated a standardised version that inquires about the same activities in all patients in two randomised validation studies. Comparison of the individualised with the standardised version revealed slightly greater responsiveness of the individualised version. In addition, self-administration led to increased responsiveness compared with interviewer administration [116, 117].

For the individualised domain, the patient is asked to report or identify the five most important activities that caused breathlessness over the previous 2 weeks. A list of 26 activities is 
offered for consideration by the patient. The patient then grades the severity of dyspnoea for each activity on a 1-7 ("extremely short of breath" to "not at all short of breath") Likert-type scale; in other words, for each of the five activities that are either individual specific or standardised, a score of 17 is allotted. The mean score across completed items is calculated and yields a score for the CRQ dyspnoea domain ranging $1-7$. In COPD patients, the scores for the dyspnoea domain of the CRQ are normally distributed [145] and they are reproducible in the short (2 days-2 weeks) and long term.

Although the CRQ has been used to measure HRQoL in RCTs evaluating pharmacotherapy in patients with COPD, in most of these studies the complete scores for the dyspnoea domain were not reported [160, 162-164]. In a study examining the efficacy of a pharmacological intervention using the dyspnoea domain of the CRQ [165], there were no significant differences among three treatment groups (salmeterol and placebo; salmeterol and ipratropium bromide; and placebo). In an observational study [36], the dyspnoea domain of the CRQ was shown to be a valid and responsive measure of acute changes in dyspnoea associated with a COPD exacerbation. In an RCT [161], patients had significant improvements in the dyspnoea domain $(p=0.02)$ after 10 days of treatment for an exacerbation of COPD with prednisone plus standard therapy (+1.69 units) compared with placebo plus standard therapy (+0.97 units).

In a similar manner to previous types of dyspnoea measurement, it is possible that cardiac disease could influence the severity of dyspnoea. However, in RCTs involving patients with COPD, those patients with clinically significant cardiac disease are typically excluded from entry into the studies.

The measurement of the dyspnoea domain of the CRQ can be used in multicentre trials [160,162-165]. For the recommended SAS version, no specific training is required. It takes $\sim 10 \mathrm{~min}$ to administer with an interviewer, compared with $\sim 7 \mathrm{~min}$ for the self-administered version $[115,116]$. For the original CRQ instrument, an interviewer, a list of 26 activities that might cause dyspnoea and Likert scoring cards are required. The interviewer should have a basic knowledge of respiratory disease and scoring on the Likert-type scale. Any cost of either version is based on the intended use of the questionnaire, as determined by McMaster University, Hamilton, ON, Canada.

\section{Ratings during exercise}

Another approach is to instruct patients to report the severity of dyspnoea while performing an exercise task, such as cycling or walking. Various exercise protocols have been used, including incremental and constant work exercise tests. There are two common methods for COPD patients to rate their dyspnoea during an exercise test: the $0-10$ category ratio (CR10) scale and VAS.

The CR10 scale incorporates nonlinear spacing or verbal descriptors of severity corresponding to specific numbers with ratio properties [166].

VAS consists of a vertical or horizontal line, usually $100 \mathrm{~mm}$ in length, with descriptors typically positioned at the extremes of the scale as anchors.
There are several advantages of using the CR10 scale rather than VAS for rating dyspnoea during exercise. First, the CR10 is open-ended as there is an opportunity to provide a rating $>10$, whereas VAS has a ceiling effect (the highest possible rating is $100 \mathrm{~mm}$ ). Secondly, the presence of descriptors on the CR10 scale allows direct comparisons between individuals or groups. Thirdly, the CR10 scale should be easier for patients to use for exercise prescription. The use of a number or descriptor on the CR10 scale (e.g. a rating of 3 or moderate breathlessness) would be more relevant as a dyspnoea target for exercise training, rather than a length in millimetres on the VAS. The CR10 scale has been combined with a continuous method for patients to rate dyspnoea [167]. With this system, the subject moves a computer mouse that adjusts a vertical bar positioned next to the CR10 scale so that patients can provide ratings "whenever there is a change in breathlessness" throughout exercise, rather than "on cue" at each minute of exercise [167-169].

In general, ratings during exercise provide different and distinct information than that obtained by clinical ratings of dyspnoea [141].

\section{The CR10 scale}

In 1982, BORG [166] developed a 0-10 rating scale constructed as a category scale with ratio properties. This CR10 scale incorporates nonlinear spacing of verbal descriptors of severity corresponding to specific numbers and ratio properties of sensation intensities. The CR10 scale provides a standard method for patients to select ratings of dyspnoea on a scale based on descriptors that correspond to specific numbers. The patient should be given specific written instructions on how to use the CR10 scale to rate dyspnoea prior to the exercise test [170]. During the incremental or constant work exercise test, the patient is typically cued to indicate a rating toward the end of each minute or each new workload. An alternative approach enables the subject to provide continuous ratings of dyspnoea ("whenever there is a change in breathlessness") by moving a computer mouse that adjusts a vertical bar positioned adjacent to the CR10 scale visible on a monitor [167-169].

With respect to frequency distribution of the CR10, there is a wide range of dyspnoea ratings among patients with COPD $[169,171]$. It is a reproducible measure in the short (i.e. 2 days2 weeks) and long term. It has been determined from factor analyses that dyspnoea ratings during exercise tests are separate and distinct from lung function and exercise capacity [145]. These ratings have also shown to be responsive to various pharmacotherapies in patients with COPD [29, 49, 50, $169,172,173]$. However, it is not recommended to be performed during exacerbations of COPD.

In multicentre studies, patients have successfully rated the severity of dyspnoea on the CR10 scale during exercise tests as part of RCTs evaluating various pharmacotherapies $[29,49,50$, $169,172,173]$. Again, similar to the other measures of dyspnoea, it is possible that cardiac disease could influence the severity of dyspnoea.

The ratings of dyspnoea on the CR10 scale are typically obtained from an exercise task, such as cycle ergometry or treadmill walking. For the continuous method for patients to rate dyspnoea, a computer with a mouse, a monitor with the CR10 scale, and the programme to operate the system are 
required. The time needed to retrieve these ratings is dependent on the duration of the exercise task. Patients need to have an initial familiarisation session to practice using the CR10 scale and exercise equipment prior to being randomised to therapy. The programme to operate the computerised continuous method for patients to rate dyspnoea is available from Psychological Applications and any fee related to its use is determined based on its intended purpose.

\section{Conclusions}

A number of clinical and physiological outcomes, such as dyspnoea, functional status and health status, are recognised as being important for the characterisation of response to treatment. Instruments used to measure dyspnoea should rely on patient-reported outcomes, be multidimensional where possible, adhere to standardised methodology and ideally be computerised. Instruments that are third-party rated may prove less compelling than validated patient-rated instruments. These recommendations should be upheld if the evaluation of dyspnoea in COPD is to provide consistently reliable data in future clinical trials [109]. Health is an abstract concept but it is possible to produce standardised health status measures that have true interval-scaling properties (i.e. the questionnaire behaves like a ruler). While there is a relationship between reduced lung function and impaired health [174], this is not sufficiently strong for spirometric measures to provide a reliable estimate of HRQoL [175]. For that reason, measurements of health status must be performed using specifically designed questionnaires. Unfortunately, recent evidence indicates the possibility of an improvement in health status in COPD patients even after a regular treatment with placebo [176], whereas a meta-analysis of the published clinical trials indicates that SGRQ is only able to differentiate between health condition and presence of COPD, but it is not a real indicator of the severity of the disease according to the severity of airway obstruction [177]. At this time, the poor correlation between changes in FEV1 and in quality-of-life scores indicates the need for using a multidimensional approach.

\section{EXACERBATIONS}

\section{Introduction}

The chronic and progressive course of COPD is often aggravated by short periods of increasing symptoms, particularly increasing cough, dyspnoea and production of sputum that can become purulent. The majority of these COPD exacerbations are caused by bronchial infection and, if frequent, have been demonstrated to have a negative impact on HRQoL in patients with COPD [178-181]. Furthermore, acute exacerbations are the most frequent cause of medical visits, hospital admissions and death among patients with COPD [182]. One of the main objectives of COPD treatment is to reduce the frequency and severity of exacerbations. Unfortunately, there is no validated diagnostic test or biomarker of exacerbations [183]; therefore, the diagnosis of an exacerbation must be based on a clinical definition that includes the most frequent symptoms observed during these episodes. Definition of exacerbations and their severity need to be standardised to allow comparisons between different interventions in different settings [184].

\section{Definition of exacerbation}

At present, there is no standardised and unanimously accepted definition of exacerbation of COPD [184]. However, four definitions are widely used.

The first definition uses a combination of three cardinal exacerbation symptoms: increased dyspnoea; sputum volume; and sputum purulence. Type I exacerbations were defined as occurring when the three symptoms were present. Type II exacerbations were defined as occurring when two of the three symptoms were present. Type III exacerbations were defined as occurring when one of the three symptoms was present in addition to at least one of the following findings: upper respiratory infection within the previous 5 days; fever without other cause; increased wheezing; increased cough; or an increase in respiratory rate or cardiac frequency by $20 \%$, as compared with baseline [185]. This definition has been widely used in clinical trials of antibiotics for exacerbations of COPD.

The second definition of exacerbations looks at the presence of the following patterns of symptoms during $\geqslant 2$ consecutive days: either two or more of three major symptoms (increase in dyspnoea, sputum volume and sputum purulence); or any one major symptom together with any one minor symptoms (increase in nasal discharge, wheeze, sore throat, cough or fever) [178]. This definition has been used in followup studies of COPD patients and, unlike the first definition, has the advantage that all exacerbations, whether reported or unreported to healthcare professionals, can be identified, increasing the exacerbation frequency. The identification of unreported exacerbations requires the use of a diary card of symptoms.

The third definition was proposed as a consensus definition of an experts' panel: a sustained worsening of the patient's condition, from the stable state and beyond normal day-to-day variations, that is acute in onset and necessitates a change in regular medication in a patient with underlying COPD [186].

The fourth definition, proposed by some pharmacological randomised clinical trials [152,187], identifies exacerbations as a complex of respiratory events (i.e. cough, wheezing, dyspnoea or sputum production) lasting $\geqslant 3$ days. However, there is no evidence that 3 days of symptoms are required to define an exacerbation. Unlike asthma, patients with COPD do not experience sudden increases in symptoms that may disappear spontaneously or with medication in a few hours or days [188]. Furthermore, delay in initiating treatment for an exacerbation may result in a longer duration of the episode [189]. Consequently, no time limit should be required to define an exacerbation of COPD.

The use of exacerbation frequency as an outcome measure in clinical trials in COPD may require the quantification of all episodes. Consequently, the definition should include any increase in respiratory symptoms over baseline. In this respect, the definitions used in existing clinical trials of bronchodilators and/or inhaled corticosteroids in COPD are appropriate. Thus, the proposed definition of exacerbation of COPD would be an increase in respiratory symptoms over baseline that usually requires change in therapy $[190,191]$. However, if this definition is used, patients must be encouraged to recognise 
exacerbations and increase their own therapy, self-manage or present to healthcare professionals for exacerbation therapy. Otherwise, the exacerbation frequency will be underestimated. For the use of this definition, it is important to know that approximately two thirds of patients are aware when an exacerbation is imminent and, in most cases, symptoms are consistent from one exacerbation to another [192].

\section{Definition of severity of exacerbations}

The effect of any given therapeutic intervention may not only be to reduce the frequency of exacerbations, but also and more commonly to reduce their severity. No validated scale of severity exists for exacerbations. Some authors have used a composite scale of symptoms to evaluate the resolution of the episode in clinical trials of antibiotics [193] or in observational follow-up studies [194]. However, to date these scales have not been validated in long-term trials of interventions in stable COPD patients. In contrast, most studies have used the intensity of the medical intervention required as a grade of severity, from self-management at home to admission to an intensive care unit [152, 154,191, 195-197]. The classification in types I-III according to criteria defined by ANTHONISEN et al. [185] is not a severity scale but a classification that indicates the likelihood of bacterial infection as cause of an exacerbation (i.e. a type I exacerbation in a mild patient may have a better prognosis than a type III exacerbation in a severe patient).

The proposed severity classification includes three categories: 1) mild, which involves an increase in respiratory symptoms that can be controlled by the patient with an increase in the usual medication; 2) moderate, which requires treatment with systemic steroids and/or antibiotics; and 3) severe, which describes exacerbations that require hospitalisation or a visit to the emergency department.

\section{Evaluating the frequency of exacerbations}

Due to seasonal variation, an evaluation of exacerbation frequency requires a period of $\geqslant 1 \mathrm{yr}$ [179]. The methods for recording exacerbation frequency as a variable have not been standardised, but various methods have been used in several clinical trials of inhaled corticosteroids and/or bronchodilators in COPD [152, 154, 190, 191, 195-198].

In observational studies of COPD patients, a skewed distribution of this variable has been found with a large number of patients having 0-2 exacerbations per yr and a small number of patients having $\geqslant 10$ exacerbations per yr [179, 194, 198]. The mean number of exacerbations is generally related to the severity of the baseline disease and the definition used, and in observational studies ranges 1-2.5 episodes per yr [179, 194, 198]. If unreported exacerbations are included, severe patients (GOLD III) have a mean of 3.43 exacerbations per yr and GOLD II patients have a mean of 2.68 per yr [194].

In the short term (i.e. weeks or months), this variable does not appear to be reproducible due to the small number of episodes per yr; the probability of repeating an episode in weeks or months is small. However, in the long term, patients with frequent exacerbations in the past have a large probability of suffering frequent exacerbations in the future [194, 199]. In COPD patients, there is also a short-term [36] and a long-term impact of exacerbations on HRQoL [178-180, 200]. The magnitude of this impact is directly related to the frequency of exacerbations [178-180, 200]. In addition, poor HRQoL is a risk factor for frequent exacerbations [179]. Numerous clinical trials have also shown that it is sensitive to treatment effect, with apparent reduction in the frequency of exacerbations [152, $154,190,191,195,197,198]$. Comorbidities, particularly cardiac diseases, are a risk factor for poor outcome of the exacerbation [201] but, to date, there is no evidence that comorbidities are a risk factor for frequent exacerbations.

With respect to the feasibility of measuring the frequency of exacerbations, simpler methods are more likely to be used in multicentre trials. A questionnaire in the form of a diary card is needed in order to capture the unreported exacerbations from patients and another questionnaire for investigators to quantify the reported exacerbations [178, 194]. The questionnaires take $<1 \mathrm{~min}$ each to complete. No additional equipment or training is required.

The statistical methodology used to calculate the annual ratio of exacerbations in a given cohort and to compare the different ratios between treatment arms in clinical trials must be described in detail, since great and significant differences have been reported when different approaches have been used [202].

\section{Evaluating the severity of exacerbations of COPD}

The methodology surrounding the use of severity of exacerbations as a variable has not been standardised. The criteria for hospital admission may vary from country to country and in different hospitals. In addition, the use of systemic steroids and/or antibiotics for exacerbations has different patterns in different areas. The severity of exacerbations is closely related to the severity of the baseline disease, i.e. severe COPD patients are more likely to be hospitalised due to an exacerbation. Therefore, distribution of severity of exacerbations parallels distribution of severity of COPD in a given population. Up to $50 \%$ of exacerbations may be unreported to the physician and managed at home (mild exacerbations), $40-45 \%$ are moderate exacerbations and $<10 \%$ are severe exacerbations [178, 194, 198, 203]. This variable is not reproducible in the short term but, in the long term, COPD patients who experience severe exacerbations have an increased risk of experiencing more severe exacerbations in the future $[194,204]$. In COPD patients, this variable does influence health outcome since severe exacerbations, which require hospital admission, do have an impact on health status [179]. Many RCTs have also demonstrated improvements in the severity of exacerbations with pharmacotherapy compared with placebo in patients with COPD [152, 154, 195, 197, 198]. It is possible that cardiac disease may influence the severity of the exacerbation. Patients with cardiac disease have an increased risk of hospitalisation due to an episode [205] and have an increased risk of mortality [206].

The severity of exacerbations can be measured in multicentre trials. This is based on medical resource utilisation observed in numerous RCTs of pharmacotherapy compared with placebo $[152,154,187,190,195-198]$. It requires the use of a questionnaire or a diary card for the patient to detect the unreported (mild) exacerbations; and a questionnaire is also used for the investigator to collect information on reported (moderate-to-severe) exacerbations [178, 194]. Both take 
$<1 \mathrm{~min}$ to complete and require no additional equipment or training costs.

\section{Conclusions}

The definition of exacerbations for clinical trials with antiinfectives should include the recognition of symptoms related to the likelihood of infectious aetiology. However, the definition used in clinical trials of pharmacotherapy in stable COPD is not restricted to exacerbations of infectious aetiology. The proposed definition of an exacerbation of COPD is an increase in respiratory symptoms over baseline that usually requires medical intervention. This definition requires the patient to be encouraged to recognise exacerbations and increase their own therapy or present to healthcare professionals. Patient diary cards are useful for recognising these episodes. The definition of severity is as follows: 1) mild, which involves an increase in respiratory symptoms that can be controlled by the patient with an increase in the usual medication; 2) moderate, which requires treatment with systemic steroids and/or antibiotics; and 3) severe, which describes exacerbations that require hospitalisation or a visit to the emergency department. The severity of exacerbations is largely based on the degree of healthcare utilisation and this definition has the disadvantage of having different criteria for hospital admission or different patterns of use of antibiotics and/or oral steroids in different settings or countries. At this time, no biological marker for the risk of exacerbation or severity exists that can be used in clinical practice or for an RCT.

\section{EXERCISE}

\section{Introduction}

In chronic cardiopulmonary diseases the ability to exercise is an important clinical outcome in its own right and a marker of other significant outcomes. Exercise tolerance is significantly impaired in many patients with COPD and is an important determinant of HRQoL [119, 207]. In COPD patients, exercise tolerance cannot be predicted by resting lung function measurements (e.g. FEV1) and exercise testing is useful in the clinical setting to assess the degree of impairment, prognosis and the effects of interventions. Exercise capacity is mostly limited by ventilatory constrains, lung gas exchange inefficiency leading to increased exercise ventilatory demand, lung dynamic hyperinflation and dyspnoea sensation [208, 209]. Deconditioning and/or malnutrition leading to peripheral muscle dysfunction and leg fatigue may also contribute significantly to reduced exercise capacity [210].

The ability of exercise to provoke breathlessness is used in the MRC dyspnoea scale to estimate symptom intensity (see section on Dyspnoea) [144] and exercise impairment is an important predictor of mortality [211, 212].

The severity and cause of exercise intolerance are best assessed by performing detailed physiological measurements in the laboratory (minute ventilation, breathing pattern, oxygen uptake, carbon dioxide production, oxygen saturation and other derived indexes; all during exercise). Simpler field tests, where it is normal simply to record the duration of exercise or the distance covered in a fixed time period, can also be utilised. Laboratory test protocols can be either incremental with a steady rise in the intensity of the workload or constant work rate tests ("endurance"), where the workload is undertaken at a fixed percentage of a previously established maximum. Incremental testing is less sensitive to interventions than endurance exercise, which is usually conducted at $\sim 75 \%$ of the symptom-limited peak oxygen uptake $\left(V^{\prime} \mathrm{O}_{2}\right.$,peak $)$ or peak work rate $[213,214]$. Field tests, such as the widely used 6-min walking test (6MWT), represent an alternative in which patients are encouraged to walk as far as they are able to for the period of the test [215]. In addition to the distance covered, it is useful to record, at the beginning and end of exercise, the intensity of breathlessness (and sometimes leg fatigue) using a modified Borg category scale or a VAS (see section on Dyspnoea), cardiac frequency and $\mathrm{Sp}_{\mathrm{p}} \mathrm{O}_{2}$. Differences in physiological adaptation to cycling and to walking have been reported in COPD in patients with increased dyspnoea and arterial oxygen desaturation during walking exercise $[216,217]$.

All exercise tests have been shown to have good validity, specificity, reliability [49, 50], predictive ability [212], discriminative ability and evaluative ability [218].

\section{Laboratory exercise testing}

Laboratory exercise testing is conducted under standardised conditions, with the recording of cardiac frequency, minute ventilation, respiratory frequency and breathing pattern, oxygen uptake and carbon dioxide production (from which the anaerobic threshold can be estimated [219]), exercise duration and maximum workload performed. Lung volumes can be measured noninvasively using optoelectronic plethysmography [220] but the most common method involves the measurement of IC (assuming the TLC to be constant during exercise). Care is needed in how this measurement is conducted [64] but in individual laboratories its reproducibility is good [65]. The intensity of respiratory sensation, breathlessness and the presence of leg fatigue are recorded using a category ratio scale (see section on Dyspnoea) at rest and at intervals during exercise (commonly at $1 \mathrm{~min}$ or just before a planned increase in workload). It is useful to note the principal symptom limiting exercise performance [216, 217]. Cardiac frequency, blood pressure, oxygen saturation and ECG are also recommended.

According to ERS and ATS/American College of Chest Physicians guidelines for cardiopulmonary exercise testing $[208,209]$, patients rest for a few minutes breathing quietly on the equipment before beginning light exercise, which increases in intensity in a ramp fashion. Cycle and treadmill exercise have been used interchangeably, although the former has been mostly used in COPD clinical studies, as the work rate for incremental and endurance tests is easier to quantify. As the exercise period should last 10-12 min, the work rate increment should be selected carefully. In COPD studies the usual rate of workload increase is $10 \mathrm{~W} \cdot \mathrm{min}^{-1}$, although slower or faster rates are possible in the very sick and the very fit patients, respectively. The maximal incremental exercise test is also utilised to determine the appropriate work rate to be used in an endurance protocol (i.e. $75 \%$ peak work rate).

The measurement of respiratory and metabolic variables together with various exercise protocols is standard, although there is no universal agreement on the rate of increase of work during exercise, which tends to be individual to the purpose of the investigation. Although the statistical frequency distributions 
of these measures have not yet been determined in the general population or in COPD patients, they are reproducible in the short term as well as in the long term, where changes can reflect disease progression. In the general population, exercise is an important determinant of overall cardiovascular mortality [221], and in COPD patients mortality has been related to $V^{\prime} \mathrm{O}_{2}$,peak in this group [212]. Many studies have also demonstrated that interventions, such as rehabilitation [222, 223], lung volume reduction surgery [224] and oxygen [225, 226] and heliox [227] administration, and treatment with bronchodilator drugs $[49,50]$ improve exercise-related indices. These have been largely demonstrated with an endurance test outcome rather than incremental testing. However, exercise testing should not be peformed during exacerbations of COPD due to the obvious patient practicalities. The presence of comorbidities, which themselves limit exercise performance, e.g. intermittent claudication or exertional chest pain, precludes exercise testing with any test modality. Coexisting occult cardiac disease can be identified during exercise from abnormalities in cardiovascular variables (i.e. cardiac frequency, ECG and blood pressure).

With respect to the practicality of implementing exercise testing, it can be performed in multicentre studies, providing there is strict quality control of data collection centrally. Studies with $\leqslant 100$ patients that have been conducted in six or more centres have now been reported with good data quality [49]. It can be expected that $>90 \%$ of COPD patients would be able to carry out exercise testing in a study. In order to undertake such testing, appropriate laboratory space is necessary. An electrically braked cycle ergometer or a treadmill with facility to operate at a gradient is required. Aditionally, an appropriate flow meter and gas analysers, which are commonly part of commercial computerised exercise-recording systems, will be needed. Ideally, any exercise test should measure a physiological variable in a breath-by-breath fashion. Testing with this modality requires 45-60 min to fully complete a test and allow the patient to rest. Training is necessary for the operators, who would normally be physiological measurement technicians, graduate students or equivalent. Costing varies from centre to centre but is not insignificant.

\section{Field tests}

Self-paced timed walking tests

Patients are asked to walk as far as they are able to on a level corridor for a set time, originally $12 \mathrm{~min}$ [228] but now usually $6 \mathrm{~min}$ [215]. They are allowed to stop if they cannot continue but are asked to resume walking when they are able to. The intensity of relevant respiratory sensation is recorded as for laboratory testing, usually at the beginning and end of the exercise period. Oxygen saturation and cardiac frequency are also recorded using a light-weight portable pulse oximeter. The principal outcome is the distance walked in either metres or feet.

In terms of standardisation and reproducibility, the use of both encouragement during the walk [229] and a circular course [230] improves test-to-test reproducibility. The reproducibility of the tests is well defined in both respiratory and cardiac populations [231, 232]. There is a consistent practice effect and at least one, and preferably more, practice walks should be conducted before reliable data can be obtained [232]. There are both floor and, in particular, ceiling effects in this form of testing. The measurement is not suitable for use in less disabled subjects with walking distances $>600 \mathrm{~m}$, as factors other than those related to disease intensity determine exercise performance. The 6-min walking distance (6MWD) is an important predictor of mortality [233] and health status [174] in COPD patients. This test is now used as a standardised outcome measure in studies of treatment of pulmonary hypertension and has been applied to patients with congestive cardiac failure. Multiple studies also confirm that treatment with lung volume reduction surgery [234, 235], rehabilitation [236], oxygen during exercise [237] and a range of bronchodilator drugs [27, 238] improve this outcome significantly. As with exercise testing, the walking test is not recommended to be performed during exacerbations of COPD or when the patient has another serious comorbidity.

The walking test can be performed in multicentre trials, provided that suitable care is taken with the instructions to the operators. The simplicity of this test can be deceptive, since its performance may be influenced by patients' fatigue when they are asked to participate in protocols involving multimeasurements. The test requires a level measured distance in a corridor, an appropriate timer and a pulse oximeter, and CR10 questionnaires. The walk itself will take $6 \mathrm{~min}$ but appropriate preparation and resting usually requires 2030 min per test. A practice walk for the patient and appropriate familiarity with the test by the operators are recommended. The test is relatively inexpensive and can be mastered by nonspecialists.

\section{Shuttle walking tests}

These tests were developed as an alternative to the unpaced $6 \mathrm{MWT}$ for use as field exercise tests in an attempt to improve standardisation and reproducibility. The patient walks around an elliptical course defined by two cones $10 \mathrm{~m}$ apart, i.e. the shuttle distance. Walking speed is externally paced by the frequency of bleeps on a pre-recorded tape. The frequency increases progressively during the walk in the incremental shuttle tests until patients can no longer match their walking pace to the bleep frequency. Cardiac frequency, oxygen saturation and symptom scores are measured. The principal outcome is the distance covered in metres or feet. The distance completed on the shuttle test strongly correlates $(\mathrm{r}=0.86)$ with $V^{\prime} \mathrm{O}_{2}$,peak measured during incremental cycling exercise [239].

This test has been adapted as an endurance protocol with the external signal frequency constant throughout the walk. The peak bleep rate is set at a value that corresponds to $70 \%$ of the maximum oxygen uptake, which is determined indirectly from a previously established relationship between cardiac frequency and shuttle distance. The principal outcome of the endurance shuttle walk test is the duration of exercise in seconds.

The shuttle walk test and its variants have been standardised and a protocol published [240, 241]. The incremental test requires an a priori familiarisation study, while the endurance shuttle walk needs an incremental test that serves to familiarise the patient with the key aspects of the protocol. In terms of reproducibility, current data (not yet published in full) suggest that the shuttle walking distance is stable over a 6-month 
period, although this is sufficiently long for exercise performance to decline as a result of disease progression. The influence of this test on health outcome has not been specifically studied; however, there are studies published in full or in abstract that suggest that this measure is responsive to pulmonary rehabilitation [242], nutritional support [243, 244] and bronchodilator drugs [245]. In addition, as with all exercise tests, the same considerations are made regarding its use during exacerbations or in the presence of coexisting diseases.

The shuttle walk test may also prove useful in multicentre studies. There has been only one small trial $(n=80)$ conducted at three UK centres, but the influence of the variability between centres on the results was not assessed. In terms of cost, the shuttle walk text is relatively inexpensive, requiring two cones separated by $10 \mathrm{~m}$, an appropriate tape recorder, together with a pulse oximeter and a CR10 questionnaire.

\section{Conclusions}

Exercise capacity is another important clinical outcome that could be measured in COPD pharmacological trials. Several methods for evaluating exercise capacity have been developed. The $6 \mathrm{MWT}$ is a relatively simple test that has been used extensively in trials to evaluate possible benefits of pharmacological intervention. More standardised tests have patients walking at a specific speed on the treadmill or performing cycle ergometry. Exercise duration, power output (in watts) and oxygen consumption (in $\mathrm{mL} \cdot \mathrm{kg}^{-1} \cdot \mathrm{min}^{-1}$ ) are also standard measures of exercise capacity.

\section{MORTALITY}

\section{Introduction}

Mortality provides the best possible outcome to measure. Reliable, relatively easy to measure and of great importance, it has been the gold standard in the evaluation of predictors and therapies. In COPD, several studies evaluated predictors of mortality. Four reports have shown improvements in mortality with COPD therapies: two trials evaluating oxygen for hypoxaemic patients [246, 247], one patient level meta-analysis trial of inhaled corticosteroids [248], and one of lung volume reduction surgery in a small subgroup of patients [218].

\section{Cause of death in patients with COPD}

Patients dying as a consequence of severe COPD do not regularly have COPD listed on their death certificates, making COPD-specific mortality a difficult outcome measure [249, 250]. Patients with advanced COPD are often recorded as dying from other causes, sometimes trivial insults, such as hospital admissions for unrelated events (e.g. fractures), or therapeutic mishaps, such as sedation, uncontrolled oxygen or maintenance treatment withdrawal. COPD is also an independent risk factor for cardiovascular deaths [251, 252]. All-cause mortality is probably the best COPD outcome measure.

\section{Adjudication of the cause of death}

The correct determination of the cause of death remains important, however, as it may help generate hypotheses regarding the mechanism by which a variable in question affects the outcome or the influence that a treatment under study may have on the outcome.
Very little has been published in the area of accurately defining the cause of death. As a matter of fact, only one study [196] was found in the COPD literature where a methodology for the adjudication of the actual cause of death was described. In contrast, adjudication committees for the cause of death and a description of their methods are frequent in cardiovascular trials $[253,254]$. The discrepancy between the original and the adjudicated cause of death in some of those studies has reached 20\% [196]. There is emerging consensus that large clinical trials evaluating death as an outcome should have an independent adjudicating committee [255] consisting of at least three members [256]. More members do not appear to increase the accuracy of classification [254].

\section{Predictors of mortality}

In table 2, the variables that represent predictors of mortality are presented along with the stage (GOLD-ATS/ERS) included in the studies and whether it predicts all-cause and respiratory mortality [5, 55, 212, 249, 257-269].

With mortality as an outcome, the variables listed represent surrogate outcomes. They could be used as surrogates for mortality, especially if their changes reflect changes in mortality. A case in point is correction of oxygen in hypoxaemic patients, which alters the mortality rate. Unfortunately, surrogates often do not show a relationship or even contradictory results when compared with important patient outcomes.

Thus, the relative strength of the variables remains difficult to ascertain. Recent data do suggest that, at least for the body mass index (B), obstruction (O), dyspnoea (D) and exercise endurance (E) index (BODE index), changes in its value after intervention confer changes in prognosis [270, 271]. More studies are needed to confirm these preliminary findings. What is clear is that variables that differ from FEV1 are

\begin{tabular}{|c|c|c|}
\hline Marker & COPD stage ${ }^{\#}$ & Cause of mortality \\
\hline BMI & I-IV & All cause/respiratory \\
\hline FEV $_{1}$ & I-IV & All cause/respiratory \\
\hline IC/TLC & I-IV & All cause/respiratory \\
\hline \multicolumn{3}{|l|}{ Exercise } \\
\hline$V^{\prime} \mathrm{O}_{2}$,peak & I-IV, tilted towards III-IV & Systemic \\
\hline 6MWT & I-IV, tilted towards III-IV & Systemic \\
\hline Dyspnoea & II-IV & All cause/respiratory \\
\hline Decreased chronic $\mathrm{Pa}, \mathrm{O}_{2}$ & III-IV & Systemic \\
\hline BODE & O-IV & All cause/respiratory \\
\hline C-reactive protein & I-IV & All cause \\
\hline
\end{tabular}

COPD: chronic obstructive pulmonary disease; BMl: body mass index; FEV1: forced expiratory volume in one second; IC: inspiratory capacity; TLC: total lung capacity; $V^{\prime} \mathrm{O}_{2}$,peak: peak oxygen uptake during cardiopulmonary exercise test; 6MWT: 6-min walking test; $\mathrm{Pa}, \mathrm{O}_{2}$ : arterial oxygen tension; BODE: body mass index $(B)$, obstruction $(O)$, dyspnoea $(D)$ and exercise endurance $(E)$ index.

\#: Global Initiative for Chronic Obstructive Lung Disease-American Thoracic Society/European Respiratory Society COPD stages. 
predictors of mortality and some, such as BODE, may be better than lung function alone.

\section{Conclusions}

Mortality remains the most important and robust clinical outcome in COPD research. Several variables different from the degree of airflow obstruction independently predict mortality in patients with COPD. Accurate determination in studies assessing mortality as an outcome requires the correct adjudication of the cause of death. The adjudication committee should consist of three individuals.

\section{SOCIAL AND ECONOMIC BURDEN Introduction}

An economic evaluation is not an outcome measure as such, but rather a specific type of analysis that compares costs and effects between two or more interventions and integrates the differences in costs and effects into a cost-effectiveness ratio. Cost is a cumulative variable that includes a wide variety of different types of healthcare utilisation and other resource use, all of which can be measured in different ways. These data are increasingly important in various jurisdictions in order to support coverage and reimbursement decisions by health authorities.

Because economic evaluations require high-quality data on outcome measures, a detailed description of the methods, data acquisition and handling and reporting requirements are relevant to the remit of the ATS/ERS Task Force.

There are several educational books and guidelines on economic evaluation [272-274] and there are reviews of economic evaluations of COPD interventions [275, 276]. The purpose of the present section is to focus on: 1) the aspects of economic evaluations that are typical or specifically relevant to COPD in the context of pharmacological trials; 2) the issues around the measurement and valuation of healthcare utilisation; 3) the measurement and valuation of productivity costs; 4) the outcomes typically used in economic evaluation; 5) the interpretation of the cost-effectiveness ratio; and 6) the epidemiological models of COPD specifically developed to estimate cost-effectiveness of COPD interventions. Most economic evaluations of COPD interventions were conducted alongside randomised clinical trials. An increasing number of cost-effectiveness analyses are using models to estimate costeffectiveness.

\section{Use of healthcare resources}

Factors to consider for measurement and valuation

Exacerbations are an important outcome in COPD, representing treatment failure and progression of the disease. Between $40-60 \%$ of medical expenditure for COPD is a direct consequence of exacerbations [277-281]. Hospitalisation, emergency department visits and unscheduled clinic visits, as well as use of rescue medication, including antibiotics, comprise the majority of these emergency treatment costs [203]. When a resource use-based definition of severity is used, costs increase with severity by definition, but costs also increase with severity when a symptom-based definition is used [282, 283]. In clinical trials, use of emergency treatments, alone or in combination with symptom and lung function data, are customarily employed to characterise an exacerbation, especially when the primary study outcome is reduction in the frequency of or the time to an exacerbation event. Routine collection of emergency treatment data can be undertaken in the field through patient or caregiver self-report. In some circumstances, automated data from clinical or billing records are more reliable and valid and can substitute self-reports.

For studies of cost impact, data on preventive pharmacotherapy, diagnostic and follow-up spirometry, oxygen use and routine office visits (downstream cost) are required to supplement data on emergency treatments in order to provide a comprehensive assessment of health resource use and costs. These data can be acquired in a similar fashion, using selfreport or automated data collection methods.

Although it is relevant to record the aforementioned use of maintenance therapy and scheduled healthcare utilisation to obtain a complete picture of the costs, it has been well established that hospital admissions are the main driver of the cost-effectiveness of most COPD interventions. However, the incidence of hospital admissions in clinical trial populations of stable moderate-to-severe COPD patients is relatively low. Hence, a very large number of patients are required to demonstrate that a reduction in the number of hospital admissions or hospitalisation days by 20 or $30 \%$ is statistically significant. As this number exceeds by far the number needed to demonstrate a difference in lung function, exacerbation rate or COPD-specific quality of life, most clinical trials do not have sufficient power to detect cost differences.

Medications are an important contributor to the total costs of COPD, but, with the exception of the costs of study medication, they are not usually an important driver of the costeffectiveness of COPD interventions. Economic evaluations that are performed alongside clinical trials are not suitable to detect a difference in costs of medications, because most medications are given as maintenance therapy and clinical investigators are often instructed to keep the dose constant during the trial. Moreover, the costs of medications to treat exacerbations, such as short-acting $\beta$-agonists, prednisone or antibiotics, are relatively inexpensive. So a relevant reduction in exacerbation rate will not immediately transfer into a relevant reduction in medication costs.

\section{Total costs versus COPD-related costs}

An important decision when calculating costs of healthcare utilisation is whether to calculate total costs or COPD-related costs. Theoretically, it is better to record all healthcare utilisation because it is unknown in advance whether the treatment under investigation may affect healthcare use for other than respiratory indications. Another reason it is better to record all healthcare utilisation is the difficulty of disentangling COPD from comorbidities that occur more frequently in patients with COPD than in patients without COPD. Conversely, a few rare but costly events unrelated to COPD or the treatment investigated, which by chance occur in one treatment group and not in the other, may influence the cost-difference in a way that does not correctly reflect treatment impact.

\section{Implications of clinical trial protocol-driven costs}

Among the disadvantages of an economic evaluation appended to a clinical trial is the occurrence of protocol-driven costs. These 
typically comprise the costs of the regularly scheduled trial visits and examinations. These protocol-driven costs are usually excluded. However, exclusion may underestimate the total costs since these trial visits may have substituted visits that would have occurred if the trial had not taken place. Conversely, due to the trial situation, patients may feel less reservation to contact their physician sooner in case of minor complaints. The latter affects both treatment groups equally. However, the substitution effect is more likely to occur in the control than in the active treatment group, as the condition of the patients in the control group may be less well controlled. Consequently, if there is a bias, it is more likely to be a bias against the active treatment group. However, the contribution of unscheduled visits to the total cost is generally small and it is unlikely that the difference in costs between treatment groups is largely affected by this bias.

\section{Productivity losses}

Measurement issues and analysis perspective

Assessment of patient travel and waiting time, disability and absence from and productivity while at work, and caregiver costs [284] comprise additional and important outcome measures in COPD. These nonmedical economic consequences of COPD comprise $\sim 50 \%$ of the overall disease burden. However, there are only a few instruments that can evaluate production losses [285].

Whether or not to include these costs into the cost-effectiveness analyses depends on the perspective of the analyses. Choosing a societal perspective implies including the costs of lost or impaired ability to work, as well as other production losses that may occur when patients can perform household or caring activities less well, or they engage in volunteer activities.

The absolute minimum that needs to be recorded to calculate productivity costs is the number of days of absence from paid work or the start and end date of the absence spell. At baseline, whether or not the patient has a paid job, how many days per week they work and how many hours per day should be recorded. This information should be updated during the trial. From that information, the mean number of working hours per day can be calculated and multiplied by the number of days of absence from work to estimate the total number of hours missed.

Possible approaches to calculating costs of productivity losses There are two different approaches to calculating the costs of production losses: the friction cost approach and the human capital approach [286]. The friction cost method is based on the idea that "the amount of production lost due to disease depends on the time-span organisations need to restore the initial production level" [286]. It is assumed that sick employees can be replaced after a period necessary for adaptation, i.e. the friction period. In the friction cost method productivity costs are calculated by multiplying the days absent from work with the value of the daily productivity, where the number of days absent from work is limited to the duration of the friction period. The human capital approach does not take any friction period into account, but estimates the cost of lost production from the first day of sick leave onwards.

In both approaches the value of the daily productivity can be approximated by the average gross daily earnings, which include the direct salaries and social security contributions payable by the employee.

\section{Outcomes in economic evaluation}

Typical outcomes and level of assessment

Economic evaluations can assess the economic impact of different types of interventions ranging from diagnostic, therapeutic or palliative interventions to the organisation of the process of care delivery or the implementation of COPD treatment guidelines. The topic of the evaluation and the decisions that need to be supported with the evidence from the economic evaluation drive the choice of the outcome measures. When the decision to be supported is at the macro level, such as the inclusion of a new treatment into the reimbursed benefit package of a health insurer, economic evaluations require the use of final outcomes, such as life-years gained, improvement in generic quality of life and QALYs. Cost-effectiveness ratios, such as costs per life-year gained or cost per QALY, allow the comparison of cost-effectiveness of interventions across different diseases but limit comparison across jurisdictions, as the value of single cost units differs across settings. For decisions at the institutional level, such as whether or not to introduce early assisted discharge when patients are hospitalised for a COPD exacerbation, it might be sufficient to measure diseasespecific quality of life, re-admission rate and mortality, in addition to other clinical outcomes. In this specific example, caregiver quality of life would also be a relevant outcome measure to include in an economic evaluation.

\section{QALYs and COPD}

Obtaining QALYs requires the use of a preference-based quality-of-life instrument or utility instrument, such as the EQ-5D or the Health Utility Index. However, these instruments have been criticised for not being sensitive to changes in COPD patients' health status. Part of this insensitivity is caused by the absence of dimensions of health that are particularly relevant for COPD in these instruments and the relative less importance for COPD of some of the dimensions that are included. For example, the pain dimension is often present, but breathlessness and fatigue are usually absent. Moreover, these utility measures do not capture the impact of exacerbations on quality of life very well. This criticism also applies to the commonly applied COPD-specific quality-of-life measures.

In COPD, the cost-effectiveness of a few interventions has been assessed in terms of cost per QALY. These interventions include lung transplantation [287-289], lung volume reduction surgery [290], mechanical ventilation [291], pulmonary rehabilitation [292, 293], smoking cessation [294], screening [295] and pharmacotherapy [296].

\section{Incremental cost-effectiveness ratio}

The cost-effectiveness ratio and its graphical analysis

In a similar manner to most new treatments, new interventions to manage COPD rarely generate net savings; that is, the costs of the intervention are not offset by the savings in other healthcare resources. More commonly, new interventions are more effective than their comparator but also more costly. This information on additional costs and effects can be combined into a cost-effectiveness ratio. A cost-effectiveness ratio is calculated as the mean difference in costs divided by the mean 
difference in outcomes (e.g. QALYs). Confidence limits cannot be applied to these differences. If they could, a negative ratio that results from dividing a negative difference in costs (i.e. savings) by a positive difference in outcomes would be treated exactly the same as a negative ratio that results from a positive difference in costs by a negative difference in outcomes, which is obviously wrong. Therefore, the uncertainty around a cost-effectiveness ratio is usually shown as a confidence region on the cost-effectiveness plane [297, 298]. A cost-effectiveness plane is an $x-y$ diagram of the difference in outcomes on the $x$ axis and the difference in costs on the y-axis. An example of a cost-effectiveness plane of drug A compared with drug B is provided in figure 1 . The quadrants of the plane show the possible combinations of positive or negative outcomes with positive or negative costs. The dots that form the confidence region can be obtained by bootstrapping. The bootstrap technique estimates the sampling distribution of the costs and effects through a large number of random draws from the original data, based on sampling with replacement [299]. For each bootstrap sample a new cost-effectiveness ratio is calculated. All these cost-effectiveness ratios are plotted on the cost-effectiveness plane, reflecting the uncertainty as a confidence region around the cost-effectiveness ratio. It can be calculated which proportion of the bootstrap replications of the cost-effectiveness ratio fall in each of the quadrants. Figure 1 shows a cost-effectiveness plane with $72 \%$ of all ratios in the north-east quadrant (drug A costs more than drug B, but is more effective), $3 \%$ of all ratios in the south-east quadrant (drug $\mathrm{A}$ is more effective at less cost than drug B), $2 \%$ of all ratios in the south-west quadrant (drug A is less costly but also less effective than drug B) and $44 \%$ of all ratios in the northwest quadrant (drug A is more costly and less effective than drug B).

\section{The cost-effectiveness acceptability curve}

Whether an intervention is cost-effective cannot be judged without information on the maximum that decision makers are willing to pay for a QALY, an exacerbation-free month or another unit of effect. Although some countries, such as the UK, disclose information on the maximum acceptable costs per QALY [300], most countries do not. As the maximum willingness to pay is unknown, the information on the costeffectiveness plane can be used to estimate the likelihood that a treatment is cost-effective at various levels of the willingness to pay. This likelihood is what is presented in a cost-effectiveness acceptability curve [301]. Figure 2 shows the acceptability curve created from the plane in figure 1. The acceptability curve presents the likelihood that drug $\mathrm{A}$ is the most costeffective of the two treatments as a function of the maximum acceptable willingness to pay for a QALY. This maximum acceptable willingness to pay is often called a ceiling ratio. The curve shows, for example, an $\sim 70 \%$ chance that the incremental cost-effectiveness ratio of drug A versus drug $\mathrm{B}$ is $<€ 10,000$. In other words, the probability that drug A is most cost-effective when decision makers are willing to pay $\leqslant € 10,000$ for a QALY is $\sim 70 \%$. The curve starts somewhat below 0.2 , which indicates the probability that treatment $\mathrm{A}$ is cost saving compared with treatment $\mathrm{B}$, and asymptotes to 0.8 , which indicates the probability that treatment $\mathrm{A}$ is more effective than treatment $\mathrm{B}$. The reading starting at a probability of 0.5 , across to the curve and down to the x-axis shows the

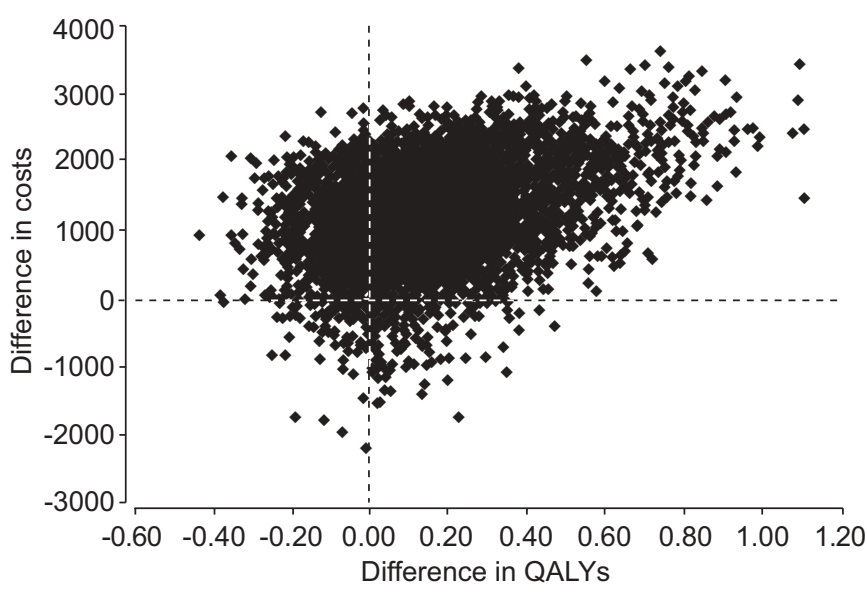

FIGURE 1. Example of a cost-effectiveness plane comparing drugs $A$ and $B$. QALYs: quality-adjusted life-years.

incremental cost-effectiveness ratio of drug A compared with $\mathrm{B}$; in this example $€ 4,118$.

\section{COPD progression simulation models}

Models may follow up on the empirical assessment of costeffectiveness alongside clinical trials, for example when there is a need to extend the time horizon of a clinical trial in order to capture all relevant economic end-points. Simulation models can be used to project disease burden or estimate costeffectiveness of interventions [277]. The Burden of Lung Disease model is designed as a burden-estimation tool for use by policy makers and local researchers. In addition, recent work describes estimates for the cost-effectiveness of COPD interventions [294, 302-305]. These models are called "state transition models" or "Markov models" [306], which simulate the progression of COPD over different stages of disease severity and model the probability to experience COPD exacerbations. This interest in COPD models is also driven by the need to adapt the model input (e.g. the prevalence distribution of COPD severity or the average length of hospital stay) before transferring the cost-effectiveness results from one country or setting to another country or setting.

\section{Conclusions}

Cost-effectiveness analyses evaluate the net changes in costs and outcomes that will result from using a new treatment in a particular group of patients, compared with an existing treatment. In particular, where the drug is more expensive, it is necessary to determine whether the increase in cost is justified by the resultant improvement in patient-centred outcomes. Unfortunately, in COPD pharmacological trials the presence of protocol-induced visits may lead to increased monitoring of patients with associated improvements in health behaviour and compliance, which may lead to an underestimation of costs compared with what would occur in a more naturalistic setting. Furthermore, the clinical trials have a double-masked design, so the results are unlikely to reflect compliance and patient preference differences that might be seen in a comparison of treatments. The relatively short time period of trials, in particular, places important limitations on the accuracy of the cost estimates, as expensive hospitalisations 


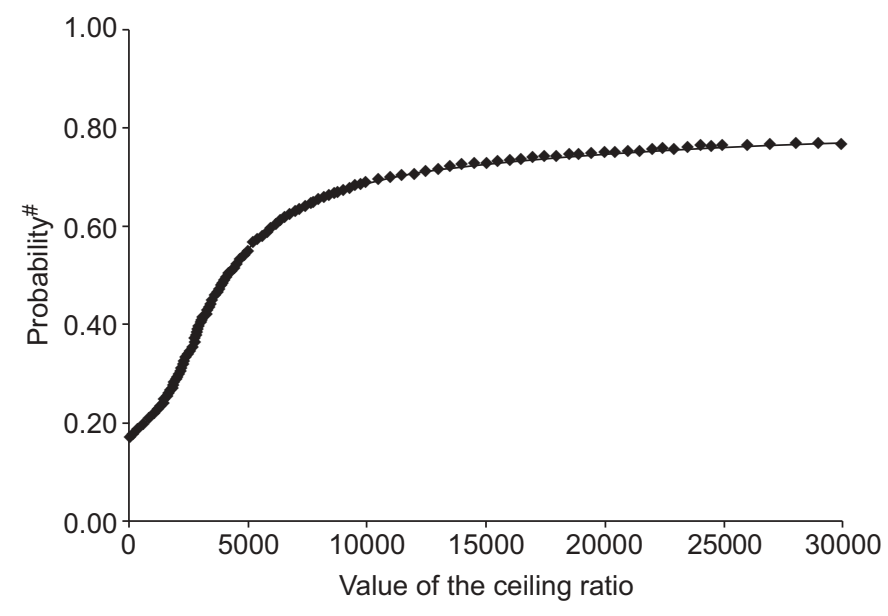

FIGURE 2. Example of an acceptability curve. ${ }^{*}$ : drug $A$ is cost-effective compared with drug $\mathrm{B}$.

may have occurred outside the study period. Moreover, few studies determine all resource use and potential downstream cost. In any case, without an evaluation of new agents using summary outcomes, such as QALYs, as the measure of effectiveness, it is difficult to gauge the value of pharmacological therapy in individuals with COPD.

\section{IMAGING}

\section{Introduction}

FEV1 is a nonspecific end-point that does not distinguish the relative contribution to airflow obstruction arising from emphysema, chronic obstructive bronchitis, asthma and bronchiectasis. As has been recently suggested, progress toward specific treatments for COPD might be accelerated by moving beyond measurements of airflow limitation to the precise diagnosis of the specific targets responsible for the airflow limitation [307]. Computed tomography (CT) imaging provides a means of accurately characterising lung parenchymal changes and the nature of the image data facilitates quantitative assessment. Although comparison between plain radiography and $\mathrm{CT}$ has shown that for clinical purposes the plain film still has an important role in the evaluation of COPD [308], CT is more sensitive than plain radiography in diagnosing emphysema, and correlates with the presence and severity of emphysema better than nonspecific physiological parameters, such as FEV1 and DL,CO/alveolar volume $[309,310]$. Longitudinal studies indicate that densitometric indices relate to the decline in FEV1 [311] but, in addition, are a more sensitive measure of emphysema progression than pulmonary function tests and health status [312-315]. Furthermore, the addition of CT evaluation of measurements of airway wall thickening has greatly contributed to the in vivo morphological study of COPD [316]. Recent data show that by quantifying both the extent of emphysema and of airway remodelling, high-resolution CT (HRCT) is useful in differentiating COPD patients who have predominant parenchymal disease from those who have predominant airway pathology $[317,318]$. The importance of determining the relative contribution of emphysema and conductive airway remodelling in individual subjects with COPD is further highlighted by a study showing that neutrophil counts in the induced sputum are significantly associated with CT indices of peripheral airway dysfunction but not with the severity of emphysema, as assessed by both CT and DL,CO [319]. Consequently, quantitative CT presents the first real opportunity to measure accurately and repeatedly in vivo lung pathological changes related to specific mechanisms of airflow limitation in COPD.

\section{General principles of CT lung parenchyma and airways analysis}

Various indices have been used to quantify lung density changes by CT. Mean lung density (MLD) is calculated by averaging the density of all pixels in the image that represent the entire lung. It has been validated by correlation with lung function tests [320323]. The percentile point is defined as the cut-off density value in Hounsfield units (HU), for which a predetermined percentage of all voxels has a lower value and, as with MLD, is also influenced by density changes in all lung structures [324, 325]. Only the fifth percentile point has been correlated with pathology [326] and lung function tests [327], although in the assessment of emphysema progression there is similar sensitivity between the 10th and 20th percentile [328]. The voxel index (VI), also referred to as Density Mask after the software program developed by General Electric Medical Systems (Milwaukee, WI, USA), or the "relative area", is defined as the proportional area under the curve of the histogram below a predetermined threshold. It is not influenced by changes in the attenuation value of voxels that remain beyond the designated threshold. Different thresholds have been applied and validated by comparative studies using pathological standards [329-333] and physiology [331, 332, 334-337].

The percentage wall area and the ratio of wall thickness to whole diameter of the right upper lobe bronchus [316, 318] or all depicted bronchi of $>2 \mathrm{~mm}$ in diameter [317] have been used to evaluate airway wall thickening. These measurements have been shown to be reliable in the assessment of the conductive airway remodelling that is characteristic of COPD, and in defining the relative contribution of emphysema and airway disease to physiological impairment. HRCT-pathological correlation has shown that CT measurements of airways with an internal perimeter $\geqslant 0.75 \mathrm{~cm}$ could be used to estimate the dimensions of the small peripheral conductive airways [338]. Recent studies have attempted to address standardisation of the methodology of airway measurements by CT [339-342].

Inherent errors in the evaluation of airways that are oriented obliquely to the axial plane may be overcome through the use of dedicated software that has been shown to be accurate in the threedimensional computation of the central axis of the bronchi and in its two-dimensional lumen and wall contour segmentation [342].

\section{Variability of quantitative $\mathrm{CT}$ indices}

With respect to scanner performance, the density of the lungs is much lower than that of water or bone and falls in a range where not all manufacturers have optimised their scanners. Some systems, for instance, have large and varying offsets at air density [343, 344]. The lung, consisting of air-filled cavities of almost zero density within nearly water-equivalent tissue, is very heterogenous and gives rise to the nonlinear partial volume effect, which may cause an underestimation of lung density [345]. 
Several physiological variables affect lung density. Lung volume is the main confounder and the physiological variable that is probably most pertinent to the reproducibility of densitometry in long-term studies. Gross lung density can vary by as much as $80-100 \mathrm{HU}$ from full inspiration to end expiration [346, 347].

At this time, the methodology has not been standardised. A number of issues remain unresolved. First, there needs to be control of or correction for variability in inspiratory level. Incorporation of a respiratory gating device consisting of a spirometer and a microcomputer has been proposed as a means of controlling ventilatory volume during image acquisition [348]. In an evaluation of this procedure, however, it was concluded that the repeatability of lung densitometry could not be improved by spirometric control [349]. A similar technique using a pneumotachometer allows the patient to breathe between acquisitions and scanning is initiated on return to a pre-selected inspiratory level [350]. An alternative approach is to standardise densitometric measurements for lung volume using a technique that acquires two volume scans at different lung volumes. The relationship between lung density and logarithmically transformed volume of air in the lungs measured from CT images is linear and thus lung density can be calculated for a specified lung volume using linear regression [328]. This approach of volume correction has been shown to improve reproducibility [351,352], but its use in long-term studies may mask some of the lung density loss secondary to emphysema-related hyperinflation. The routine use of these volume-control methods in densitometric studies remains contentious [350-355].

Consistent measurements are most likely to be achieved at maximum inspiration [353] because variation in CT lung density is lowest at full inspiration [356]; in cooperative patients, breath-holding at maximum inspiration is most reproducible [357]. A study aimed at investigating the relationship between HRCT lung attenuation measurements (employing spirometric lung volume control), pulmonary dysfunction and dyspnoea severity in patients with COPD [358], has shown that pulmonary dysfunction in COPD cannot be assessed by a single modality of lung attenuation measurements. In particular, the inspiratory level at which spirometrically gated measurements of HRCT lung attenuation are acquired influences the relationship with physiological measurements and dyspnoea perception in COPD: inspiratory measurements assess the extent of emphysematous tissue loss, expiratory measurements may reflect airflow limitation and lung hyperinflation with attendant dyspnoea perception [358].

Secondly, there are issues with the optimum image acquisition protocol. CT numbers are recognised to be unreliable [359] and are dependent on scanner type, model, object positioning within the scanner gantry and various physical factors (e.g. kilovoltage, current-time product, slice thickness and reconstruction algorithm) [343, 359-361]. Furthermore, it is recognised that spatial uniformity of CT numbers over the entire subject area may only be achievable for certain combinations of parameters [362].

The optimum acquisition protocol remains contentious. Scanner settings for optimal visual resolution and density resolution [363] are mutually exclusive and, although a standardised densitometry protocol has been developed that gives comparable results in different CT scanners [361, 364], this is at the expense of visual interpretation.

Finally, delineation methods are perhaps the most important component of imaging software. Semi-automated imageprocessing programs, such as the "seeded-region growing" method [365], reduce interobserver variability compared with the manual outlining of complex structures [366]. Software incorporating an internal calibration step may allow correction of errors in scanner calibration [344, 360, 367] and the generation of audit trails for regulation and electronic security ensure integrity [368].

There is no convincing evidence with regard to whether analysis of the whole lung is superior to single or limited slice assessment. Studies based on visual point-counting scores [369] suggest that adequate assessment cannot be obtained from one lung slice alone, although limited evidence from densitometric studies [328, 370] suggests that similar results may be obtained for the whole lung and single or limited slice analysis.

The optimum densitometric index for use in longitudinal trials remains contentious. The MLD is subject to noise [364] and is less sensitive to progression than other indices [371]. The sensitivity of the VI is influenced by threshold [328, 344], whereas the percentile point is less dependent on the choice of percentile [328, 372]. Other techniques have been described but are less well validated than the aforementioned methods [373-375].

The normal range of lung density has been determined in relationship to age and height [327, 376, 377]. This supports the sensitivity of changes in disease that are independent of either age or height [326]. However, more recent studies have demonstrated age-related changes in both airspace dimensions [378-380] and CT lung density measurements. CT densitometry is reproducible in the short term [314, 343, 351-353, 381, 382] and also likely in the long term [344, 367].

During exacerbations of COPD, accurate and precise measurements of emphysema cannot be performed. Lung density will be altered by the influence of changes in airways resistance on gas trapping and by the presence of interstitial changes resulting from infection. In addition, increased dyspnoea will influence the ability to breath-hold. In the case of coexisting illnesses, lung density will be altered by respiratory (e.g. pneumonia, pulmonary embolism, asthma, interstitial lung disease) and nonrespiratory (e.g. cardiac failure and other causes of pulmonary oedema) comorbidities.

With regard to patient safety, CT examination does entail exposure to ionising radiation. Historically considered to be a high-dose examination [383], modern scanners and protocols have allowed great reduction in effective dose delivery without loss of fidelity. Conventional HRCT (1.5-mm images at 10 -mm intervals with $140 \mathrm{kVp}$ and $175 \mathrm{mAs}$ ) delivers an effective dose of $0.98 \mathrm{mSv}$, which is $\sim 12$ times that of a posteroanterior and lateral chest radiograph [384]. Volumetric protocols entail several times this dose but the use of low-dose multidetector imaging, employing tube currents as low as 
$8 \mathrm{mAs}$, reduces the dose to below that recommended in studies of mild-to-moderate risk (1-10 mSv) [385].

The exposure to ionising radiation with CT scanning causes concerns about the risk/benefit ratio, but estimates of this ratio can only be approximate. The damaging effects of ionising radiation are assumed to follow a linear dose-effect relationship and estimates of risk are largely extrapolated from data obtained from extremely high-dose exposure [386]. The so-called "linear-no threshold" hypothesis [387] allows estimation of risk by extrapolation and rightly adopts a cautious stance towards safety. Nevertheless, this hypothesis has been questioned [388] and the explanation of risk may be more relevant and more meaningful to the lay person if expressed as multiples of the natural background radiation using the background-equivalent radiation time unit [389]. The risk/ benefit of using CT scanning in patients exposed to ionising radiation in the absence of a clinical indication underlies the need of standardising the use of low-radiation dose protocols in the context of COPD clinical trials [390]. Conversely, the lifetime mortality risk from cancer of an average 50-70-yr-old COPD patient after exposure to a low-dose chest CT is sufficiently low $[390,391]$ to justify the use of the technique in carefully controlled studies aimed to: 1) define the in vivo lung morphological changes underlying airflow limitation; 2) identify different clinical phenotypes of the disease; and 3) understand its natural history more clearly and the possible effects of preventative and pharmacological intervention. The risk/ benefit balance of CT studies in the absence of routine clinical indication is compounded by the lack of consensus on whether the risk of low levels of exposure $(<100 \mathrm{mSv})$ can be extrapolated from the complications arising from extreme levels of exposure, such as nuclear explosions or accidents.

\section{Conclusions}

Although CT densitometric evaluation allows the measurement of the progression of emphysema and the evaluation of airway wall thickening, a number of concerns have been raised regarding its use in COPD clinical trials. These mainly relate to the unresolved issues of the repeated exposure of patients to ionising radiation and the high costs involved in its frequent use. Another issue is that this methodology has not been fully validated yet. CT densitometric evaluation could be limited to a select group of patients in which the impact of treatment on airway remodelling and the progression of emphysema can be potentially studied.

\section{NONPULMONARY MARKERS \\ Introduction}

There is a growing realisation that COPD is a multiorgansystem disease. In particular, there is accumulating evidence that the skeletal muscles do not function normally, contributing to exercise intolerance. This is important because skeletal muscle dysfunction may well be a remediable source of exercise intolerance [392]. The fat-free mass (FFM) is also a significant determinant of exercise capacity in patients with COPD. With the recognition that extra-pulmonary aspects of COPD are important, standardised markers of skeletal muscle function and lean body mass must be considered. Loss of skeletal muscle mass is the main cause of weight loss in COPD, whereas loss of fat mass contributes to a lesser extent [393].

\section{Body weight and FFM}

BMI is a measure of body weight corrected for body height (body weight $\times$ height $^{-2}$; in $\mathrm{kg} \cdot \mathrm{m}^{-2}$ ), whereas the FFM index (FFMI) is a measure of FFM corrected for body height $\left(\mathrm{FFM} \times\right.$ height $^{-2}$; in $\left.\mathrm{kg} \cdot \mathrm{m}^{-2}\right)$ [394].

These measures have been standardised. BMI is widely used in health and disease as a measure of body weight adjusted to height [266]. FFM includes all compartments of the body except fat mass. It consists of muscle tissue, bone tissue and body fluids, and it can be easily measured using bioelectrical impedance analysis. More sophisticated measures are dual Xray analysis or deuterium and bromide dilution [394-400]. Statistical frequency distributions have been established for both measures. For BMI and FFMI in the general population and in the COPD patient population, the World Health Organization and COPD researchers have specified a number of ranges that represent certain nutritional states, as outlined in table 3 [261, 266, 399, 401]. Measuring BMI is highly reproducible when using the same equipment. In the case of FFMI, it is also reproducible but dependent on the method used [398, 402].

In the general population, these measures have been found to influence health outcome. Decreased BMI and FFMI are associated with impaired physical functioning. Obesity correlates with increased cardiovascular comorbidity and diabetes mellitus, but the shape of the association is not linear [403]. In COPD patients, a decreased BMI and especially a decreased FFMI are associated with impaired muscle function, exercise capacity and health status and with decreased survival [261, 266, $397,404,405]$. BMI can be increased by diet or nutritional supplementation [244, 406-408], whereas FFMI can be increased by training (rehabilitation) [409] and/or anabolic therapies, such as anabolic steroids [410]. During exacerbations of COPD, BMI and FFMI can be measured especially using bioelectrical impedance analysis [411]. However, it must be taken into account that fluid shifts can take place during exacerbations, which might affect FFM measurement. FFM depletion and involuntary weight loss are associated with an enhanced systemic inflammatory response [412-414]. Osteoporosis is commonly associated with FFM depletion [415].

The variables BMI and FFMI can easily be used in multicentre trials, with the restriction that the same equipment should be used throughout the centres. Measurements of these variables require a height rod, a weighing beam scale and bioelectrical impedance equipment. The process takes only $5 \mathrm{~min}$ and involves little training or cost.

\section{Measurement of quadriceps muscle function}

Quadriceps muscle weakness has been observed in patients with COPD [416, 417] and has been related to exercise intolerance [418], utilisation of healthcare resources [419] and survival in patients with moderate-to-severe COPD [420]. Therefore, treatment of quadriceps muscle weakness is very important in the management of patients with moderate-tosevere COPD [421-423].

Many different techniques have been used to measure quadriceps muscle weakness in clinical trials. Volitional and nonvolitional quadriceps muscle force can be measured. 


\begin{tabular}{|c|c|c|c|c|c|}
\hline \multirow[t]{2}{*}{ Nonpulmonary markers } & \multirow[t]{2}{*}{ Nutritional state } & \multicolumn{2}{|c|}{ General population } & \multicolumn{2}{|c|}{ COPD population } \\
\hline & & Females & Males & Females & Males \\
\hline \multirow[t]{3}{*}{$\mathrm{BMI} \mathbf{k g} \cdot \mathrm{m}^{-2}$} & Depleted & $\leqslant 18.5$ & $\leqslant 18.5$ & $\leqslant 21$ & $\leqslant 21$ \\
\hline & Obese & $30-40$ & $30-40$ & $30-40$ & $30-40$ \\
\hline & Morbidly obese & $>40$ & $>40$ & $>40$ & $>40$ \\
\hline \multirow{2}{*}{$\mathrm{FFMI} \mathbf{k g} \cdot \mathrm{m}^{-2}$} & Depleted & $\leqslant 15$ & $\leqslant 16$ & $\leqslant 15$ & $\leqslant 16$ \\
\hline & Normal & $>15$ & $>16$ & $>15$ & $>16$ \\
\hline
\end{tabular}

Isometric assessments involve patients sitting with their hip in $90^{\circ}$ flexion with a lever connected to the lower leg by a strap (with two fingers above the lateral malleolus). The lever has to be moved towards the knee angle of interest (e.g. $60^{\circ}$ knee flexion) and the patient is then asked to extend the leg with as much force as possible for 4-6 s.

In COPD patients, no standardisation of this technique has been conducted. With respect to reproducibility in the short term, there have only been a few studies with small samples of patients to suggest that this is indeed the case [424]. In terms of the influence of quadriceps muscle function on the health outcome of COPD patients, it is known that patients with moderate-to-severe COPD who had been admitted to hospital at least twice in the year prior to the study had significantly lower isometric quadriceps muscle force than those without hospital admission [419]. However, the actual cost of healthcare resource utilisation was not studied. Quadriceps muscle function appears to be a sensitive measure of treatment effect attributable to specific lower limb training, and multiple international clinical trials, including patients with COPD, have used a computerised dynamometer to assess isometric quadriceps muscle force before and after an exercise training programme [421]. Limited data are available with regard to muscle function testing in pharmacological intervention studies. Improvements in muscle bulk and strength are reported after hormonal replacement therapy in elderly males and COPD patients [425, 426].

In the case of isokinetic assessments, COPD patients sit with their hip in $90^{\circ}$ flexion and a lever is connected to the lower leg by a strap (with two fingers above the lateral malleolus). The lever has to be moved from $90^{\circ}$ to $0^{\circ}$ knee flexion with as much force as possible; gravity will bring the leg back into the starting position. This can be repeated 15-30 times to assess quadriceps muscle endurance [417].

Femoral nerve magnetic stimulation requires that patients lie on a specially modified couch with their knees bent at $90^{\circ}$. The ankle on their dominant side is placed in an inextensible strap connected to a strain gauge. The signal is amplified and passed to a personal computer running the software program LabVIEW (Instron Deutschland $\mathrm{GmbH}$, Darmstadt, Germany). Stimulation of the femoral nerve is carried out using a custom-made double 70-mm branding iron design coil connected via a Y-connector to two Magstim 200 Mono Pulse electromagnets (Magstim Co. Ltd, Whitland, UK). The output of two magnets combined in this fashion is equivalent to $120 \%$ of the output of a single unit. Stimulations are delivered spaced $\geqslant 20 \mathrm{~s}$ apart to avoid twitch-on-twitch potentiation.

\section{Conclusions}

The observed relationships between weight loss, muscle wasting and muscle weakness [394, 427], which are independent of FEV1, as well as the known close relationship between respiratory muscle weakness and dyspnoea, indicate the importance of adequately recording and reporting all patient characteristics in sufficient detail during pharmacological trials. This enables investigators to better understand the physical status of their patients, particularly the state of their peripheral muscles in the context of COPD. Unfortunately, BMI and FFMI are the only standardised measures available. Measurements of quadriceps muscle function are not yet standard, mainly due to the expense and need for a trained operator.

\section{MINIMAL IMPORTANT DIFFERENCE \\ Introduction}

With the increased interest in COPD and increasing number of reports of clinical trials, physicians are faced with the almost daily challenge of evaluating published reports of therapies for this disorder. Evaluating the clinical significance of such studies requires a firm grasp of statistics. The concept of minimal clinically important difference (MCID) in outcomes of therapy for patients with COPD has been proposed as a tool to assist clinicians and researchers in understanding the results of clinical trials. The minimal important difference (MID) has been defined by a group of clinical epidemiologists at McMaster University as "the smallest difference in score in the outcome of interest that informed patients or informed proxies perceive as important, either beneficial or harmful, and which would lead the patient or clinician to consider a change in management." The description of the MID precludes making MID estimates for outcomes that are remote from those important, in themselves, to patients, such as spirometry or laboratory exercise capacity. Further, the definition suggests that only if there were reasons to question the reliability or accuracy of data from patients could proxies be relied on to provide estimates of the MID [428-430]. 
The MID should optimally be determined in a population of subjects similar to that in which the MID is to be applied. Thus, the MID for various measures to be used in outcome studies of COPD should be determined from populations with COPD. The severity of the population and homogeneity of the population in which MID is estimated are also important factors to consider.

There are three basic methods for estimating MID: 1) statistical- or distribution-based methods that focus on the variance and distributional properties of scores in an untreated population of patients with the disease of interest; 2) panelbased estimates from healthcare professionals and patients; and 3) external- or anchor-based methods, which compare changes in the outcome of interest to other clinically important outcomes. Statistical methods for estimating MID include a half SD, SE of measurement (the product of the SD and the square root of one, i.e. reliability of the measure), effect size (the average change divided by the baseline SD) and standardised response mean (the average change divided by the SD of that change) [431-433]. The original method to determine the MID relied on anchor-based approaches, while panel-based methods are infrequently used. LEIDY and WYRWICH [434] have recently suggested "triangulation methodology" to describe the need to consider all three estimation methods along with expert input to identify a final MID. In addition, the uncertainty around the point estimate for the MID should be considered and provided.

MID estimates for some outcome measures in studies of COPD are summarised in table 4.

\section{Health status and HRQoL}

\section{St George's Respiratory Questionnaire}

Professional opinion-based MID

Physicians who were experts in respiratory disease were asked to make judgments about the magnitude of differences in exercise capacity, shortness of breath, wheeze, cough and depression, variables of importance in patients with COPD [444]. The magnitude of change felt to be clinically significant was assessed by clinicians and the results were applied to making an estimate of the MID for the SGRQ [445]. The resultant 3.9-unit MID estimate was similar for SGRQ total and impact scores [444]. In a clinical therapeutic trial, a 4.2-unit SGRQ change was associated with a minimum clinician rating of improvement [435].

\section{Patient opinion-based MID}

In a 16-week controlled investigation of salmeterol in COPD [446], subjects were asked to rate the magnitude of treatment effect. The smallest possible treatment improvement correlated with an SGRQ of $\sim 4$ units.

\section{External measure-based MID}

In a study of the effects of pulmonary rehabilitation, the SGRQ was compared with another disease-specific health status measure, the CRQ [447]. Using the MID for the CRQ, it was estimated that the MID for SGRQ total score (95\% CI) was 3.05 (0.39-5.71) units. The SGRQ has also been related to shortness of breath. A change in one grade of the MRC dyspnoea scale from 5 (dyspnoea leading to inability to leave the house) to 4

\section{TABLE 4 Suggested minimal important differences (MIDs) of commonly used outcomes in chronic obstructive pulmonary} disease (COPD) trials

\section{Respiratory-specific health status and HRQoL}

St George's Respiratory Questionnaire

Chronic Respiratory Questionnaire

\section{Dyspnoea}

Transition dyspnoea index

UCSD dyspnoea questionnaire

Borg scale of perceived dyspnoea

Visual analogue scale of dyspnoea

\section{Lung function}

Forced expired volume in one second

\section{Exercise}

Maximal exercise test ${ }^{\#}$

Submaximal exercise endurance test

6-min walking test

Health utility

Quality of Well-Being scale

Feeling thermometer

Exacerbations of COPD

$\begin{array}{cc}\begin{array}{c}4 \text { units } \\ 0.5 \text { units for the average score on each domain }\end{array} & \text { Jones [435] } \\ & \text { SCHUNEMANn [428 } \\ 1 \text { unit } & \text { MAHLER [436] } \\ 5-7 \text { units } & \text { RiES [437] } \\ 2 \text { units } & \text { RiES [437] } \\ 10-20 \text { units } & \text { RiES [437] }\end{array}$

$100-140 \mathrm{~mL}$

$10 \mathrm{~W}$

SUTHERLAND [438]

$1.25 \mathrm{~min}$

$37-71 \mathrm{~m}$

WISE [439]

0.03 units

KAPLAN [440]

5-7 units on 100-point scale

WOUTERS [441]

1 exacerbation per yr, 22\% change
Calverley [442]

These values are provided for informational purposes and should be considered estimates with tolerance levels rather than definite cut-off values. HRQL: health-related quality of life; UCSD: University of California, San Diego. ${ }^{*}$ : in the context of lung volume reduction surgery as an intervention. Modified from [443]. 
(significant dyspnoea but able to leave the house) correlated with an SGRQ change of 3.9 units [144].

\section{Suggested MID}

An MID (range) of $\sim 4(2.4-5.6)$ units in the SGRQ is supported by published studies.

\section{Chronic Respiratory Questionnaire}

The reliability of CRQ has been studied. Test-retest reliability intra-class correlation coefficients of $0.73-0.95$ and internal consistency reliability ranging $0.53-0.90$ (Cronbach's alpha) have been reported [117, 165, 448-451].

Statistical MID estimate

In 471 outpatients with COPD, WYRWICH et al. [118] found the SE MID estimate for CRQ to be 0.5 units. Others have reported MID estimates using the SE approach ranging 0.37-0.62 units [428].

Effect sizes have also been calculated from a study of 51 patients undergoing pulmonary rehabilitation. Effect sizes of 0.5 were found with a CRQ dyspnoea score of 0.61 units, CRQ fatigue score of 0.67 units, CRQ emotional function score of 0.60 units and CRQ mastery score of 0.60 units $[116,447]$.

\section{Panel-based MID}

WYRWICH et al. [452] used a panel of nine expert general physicians and specialists to estimate MID. The results of the research indicated available estimates of the MID of $\sim 0.5$ units.

\section{Anchor-based MID}

In a longitudinal study of a group of patients with COPD [453], patients were asked to assess their degree of change on subsequent visits on a global rating scale and the results were compared with the CRQ. Within-patient global ratings suggested mean CRQ domain MIDs of $0.43-0.64$ and ranges $0.28-0.80$

In a study of between-patient global ratings [454], subjects discussed and compared their problems on the CRQ to those of other patients. The MCID in CRQ domains ranged 0.09-0.87 with a pooled $95 \%$ CI $0.32-0.53$.

\section{Suggested MID}

An MID for the CRQ in the range of $\sim 0.5$ units is supported by numerous published investigations [428].

\section{Dyspnoea}

\section{Transitional dyspnoea index}

BDI and TDI (with TDI indicating change in response to an intervention) have been widely used in clinical studies of COPD to measure shortness of breath. The instruments are interviewer-administered and good reliability $(\mathrm{r}=0.75)$ has been demonstrated in a study of 25 patients with COPD and different interviewers [148].

\section{Statistical MID estimate}

TDI cannot be analysed in an untreated population since the instrument is only used in response to an intervention. Some of the largest changes in TDI in response to a pharmacological therapeutic intervention in COPD have been shown with tiotropium. An SD of 2.4 in one study of tiotropium provides a statistical MID estimate of 1.2 units [149].

\section{Professional opinion-based MID}

The developer of the TDI indicated that expert physicians suggest an MID of 1 unit [436].

\section{External measure-based MID}

The Physician's Global Evaluation (PGE) has been used as an external measure to compare with changes in TDI in a total of 921 subjects with COPD in therapeutic studies of tiotropium. WiTEK and MAHLER $[149,455]$ found that a 1-unit change in TDI corresponded to minimal change in the PGE. Subjects who had a $>1$-unit change in TDI had better health status, as assessed by the SGRQ, fewer COPD exacerbations, and used less rescue short-acting $\beta$-agonists.

\section{Suggested MID}

There is sufficient evidence to suggest that the MID for the TDI is 1 unit.

\section{Exercise}

6-min walking test

Of the various measures of performance of activity, the 6MWT and the incremental shuttle walk test have been studied most extensively in patients with COPD. There is more information available on the 6MWT but less data available on the shuttle walk test upon which to base an MID. The reproducibility of the $6 \mathrm{MWT}$ has been very good and the coefficient of variation has been reported to be $\sim 8 \%[215,232,456,457]$. However, when subsequent tests are performed there appears to be a definite improvement that may be due to a learning effect [230]. Additionally, factors such as encouragement and course layout have been shown to affect the results [230]. The ATS has published standards for the 6MWT and rigorous application of the suggested standardised technique for conducting the test may assist investigators in reducing variability of the test in clinical studies [458]. The 6MWT also correlates with pulmonary function (typical correlation coefficient of 0.5-0.6), dyspnoea and, to a smaller degree, with HRQoL [458-461].

\section{Statistical MID estimate}

The National Emphysema Treatment Trial (NETT) reported the results of the $6 \mathrm{MWT}$ on a very large number of subjects $(n=761)$ with severe pulmonary dysfunction and CT-documented emphysema [230]. WISE and BROWN [439] used the data from the 470 subjects who had repeated walking tests, with the second test $23 \mathrm{~m}$ longer, and calculated an intra-class correlation of 0.88 and reliability coefficient of 0.63 [439]. Using this approach, the MID was estimated as $80 \mathrm{~m}$. Using 6MWT SD of $90 \mathrm{~m}$ in all 761 subjects in the NETT, the half SD estimate for MID was $47 \mathrm{~m}$.

\section{Patient opinion-based MID}

REDELMEIER et al. [462] studied 112 patients with severe COPD, of which $50 \%$ were female. Patients were asked to rate their change in 6MWT over a period of months. There was a poor correlation between patient perception and actual walk distance, and this was attributed to poor memory of prior walk distance. Patients were asked to rate their change to differences in other patients using the global ratings of change, 
indicating the magnitude of the change they perceived. The smallest difference in walk distance $(95 \% \mathrm{CI})$ that patients were able to perceive was $54(37-71) \mathrm{m}$.

\section{Suggested MID}

The ATS guideline on 6MWT [458], the report of REDELMEIER et al. [462], the information from the NETT [230], and a recent summary by WISE and BROWN [439] support a 6MWD MID range of $54-80 \mathrm{~m}$.

\section{Constant work rate tests at submaximal exercise intensity}

Constant work rate tests performed at a submaximal exercise intensity are being increasingly used as an outcome measure in studies of COPD, largely because they have the additional advantage of indicating mechanisms leading to improved exercise. However, only a few recent studies use this measure $[49,225,463]$. The outcome of constant work rate tests at a submaximal exercise intensity is the duration of the exercise (min) that patients can perform. As recently emphasised by CASABURI [464], methodological issues may limit the interpretation of utility of this measure. The choice of initial exercise intensity is a major factor in the increase in exercise duration that can be seen on repeated testing. If the baseline exercise intensity chosen is too low, then subjects may have an almost unlimited duration of exercise following a therapeutic intervention.

\section{Statistical MID estimate}

O'DONNELL et al. [49] performed submaximal exercise tests on 187 subjects with severe COPD and hyperinflation. Mean FEV1 was $41 \%$ pred, TLC was $119 \%$ pred and RV was $198 \%$ pred. Submaximal exercise was performed at $75 \%$ of maximal work assessed on incremental cycle ergometry. Mean \pm SD baseline exercise duration prior to treatment was $492 \pm 290 \mathrm{~s}$. A half SD MID estimate based on baseline exercise capacity is $145 \mathrm{~s}$ (2.4 min). Improvement following tiotropium was $105 \mathrm{~s}$ greater than with placebo for a modest effect size of 0.36 (mean change divided by baseline SD).

OGA et al. [213] evaluated three types of exercise tests (submaximal exercise test, 6MWT and incremental cycle ergometry) in response to oxitropium in patients with COPD. The endurance exercise test was performed at $80 \%$ of maximal work achieved during an incremental cycle test. In 42 consecutive male COPD patients with a mean FEV1 of $42 \%$ pred, mean \pm SD baseline submaximal exercise endurance time was $189 \pm 92 \mathrm{~s}$, half that seen in the study by O'DONNELL et al. [49]. Thus, the half SD MID estimate based on this study is $46 \mathrm{~s}$. The mean change following oxitropium was $34 \mathrm{~s}$, with a calculated effect size of 0.37 .

\section{External measure-based MID}

There has not been a rigorous evaluation of external-based MID estimates for submaximal exercise tests, and investigations of exercise performance have not simultaneously evaluated other outcomes. For example, the study by O'DONNELL et al. [49] was an RCT, and the average improvement following tiotropium compared with placebo after 42 days of treatment was $105 \mathrm{~s}$ (1.75 min). This investigation did not assess other outcomes, but in other studies of bronchodilators, quality-of-life outcomes have reached the threshold for MCID. In addition, studies of oxygen therapy and exercise training have shown improved submaximal exercise performance in some studies, while from other studies it appears that these same interventions improve HRQoL.

\section{Suggested MID}

From this discussion, the MID range for submaximal exercise endurance time on a cycle ergometer may be 46-105 s (0.77$1.75 \mathrm{~min}$ ). In a recent review, CASABURI [464] suggested an MID of $105 \mathrm{~s}$ (1.75 min). Further investigation of the MID for submaximal exercise is clearly warranted.

\section{Maximal exercise test}

Maximal cardiopulmonary exercise tests have infrequently been used as outcome measures in clinical studies of patients with COPD. However, this laboratory test is familiar to many pulmonary physicians and has the advantage of assessing the physiological limitations to exercise, as well as providing an objective measure of exercise performance. Test-retest reliability has been reported by Cox et al. [465] as good, with a reliability coefficient of 0.96 .

\section{Statistical MID estimate}

In NETT, maximal exercise performance was used as a primary outcome measure [218]. Using the half SD estimate in NETT demonstrates a 10.5-11.1-W MID; however, the SE approach resulted in an MID of only $0.9 \mathrm{~W}$ [438].

\section{Professional opinion-based MID}

Prior to dissemination of the results of NETT, the investigators were asked their opinion of an MID in the context of lung volume reduction surgery. A value of $10 \mathrm{~W}$ was chosen by the investigators as the MID.

\section{External measure-based MID}

In NETT, the mean change in exercise capacity at $2 \mathrm{yrs}$ in patients receiving lung volume reduction surgery compared with patients receiving medical therapy was $10.9 \mathrm{~W}$. In the cohort not at risk of short-term mortality, there was also a significant improvement in HRQoL, as measured by the SGRQ [218].

\section{Suggested MID}

In the context of lung volume reduction surgery, $10 \mathrm{~W}$ may be the MID for maximal exercise workload. However, an MID for other interventions has not been established.

\section{Lung function and FEV1}

The universally used measure of lung function in clinical studies of COPD is FEV1. However, this measure of pulmonary function, currently recognised as only one of the key components necessary to fully characterise patients with COPD, has statistically significant but weak correlations with other patient-centred outcomes, such as dyspnoea [436]. Therefore, FEV1 is only one (and an imperfect) method of assessing outcomes that are important to patients in more recent studies in patients with COPD. Despite its widespread use and the large number of clinical investigations that have simultaneously measured FEV1 and other patient-centred outcomes, there has been relatively little effort to determine an MID for FEV1. Furthermore, the definition of the MID calls into question the value of pulmonary function measures as being important for patients or leading to change in management. Nevertheless, as recently reviewed by DonOHUE [466], an 
MID for FEV1 of $100 \mathrm{~mL}$ can be suggested. However, a more rigorously defined MID for FEV1 is needed. A single MID estimate may be difficult to establish for several reasons. First, baseline severity of the underlying disease assessed by FEV1 is likely to be important. In this regard, change in FEV1 after bronchodilator therapy is less marked in terms of absolute change in FEV1 in patients with lower baseline lung function. Secondly, some patients have a greater degree of response to short-acting $\beta$-agonists, which may be associated with a widely variable response to other therapeutic agents. Thirdly, FEV1 has been used as an outcome at different time-points after therapy. FEV1 can be a short-term outcome measuring peak response over minutes to hours, a longer-term outcome over hours or days, as a "trough" response prior to the next treatment dose, and as a very long-term outcome over years. Fourthly, a recent study [467] indicated a greater variation in spirometry performed in clinical practice settings compared with pulmonary function laboratories.

\section{Statistical MID estimate}

Three spirometry manoeuvres must be performed for a test to be acceptable. The recent ATS/ERS spirometry standards note that at a single evaluation the two largest FEV1 values must be within $<150 \mathrm{~mL}$ for an acceptable test [7]. This standard is also suggested by the study by ENRIGHT et al. [468], which showed $90 \%$ of 18,000 patients were able to reproduce FEV1 by $120 \mathrm{~mL}$ during a single test. However, patients with moderate-tosevere pulmonary dysfunction, demonstrated higher variability in terms of FEV1 \% pred but less variability when assessed as absolute volume (42-58 mL). In the Lung Health Study [469], spirometry performed 17 days apart showed an average absolute difference between the two tests of 110-123 mL. This reported variability of FEV1 within a single test and over a short period of time would suggest that a minimal detectable FEV1 difference over time in response to an intervention might be $\geqslant 110-150 \mathrm{~mL}$ in patients with less severe disease, but may be lower in patients with more severe pulmonary dysfunction.

Further information can be obtained using the statistical approach to estimating MID from studies of large populations. The largest study of reproducibility of spirometry over a short term was reported from the Lung Health Study [469], where spirometry was repeated after 17 days. Spirometry was performed 17 days apart in 5,885 subjects with a mean postbronchodilator FEV1 of $2.75 \mathrm{~L}$ (78\% pred). In this population, with only mild COPD, the coefficient of variation of FEV1 was $4.1-4.9 \%$. The SD of FEV1 values seen in this untreated population at entry was $620 \mathrm{~mL}$. Using the half SD approach to FEV1, the MID estimate from the Lung Health study [469] was $310 \mathrm{~mL}$.

However, MID estimates based on patients with near-normal lung function may not be appropriate for patients with more severe disease. For example, in a recently reported study of 526 COPD patients enrolled in a clinical trial of $N$-acetylcysteine [187] FEV1 was $1.65 \mathrm{~L}$ (57\% pred) with an SD of $380 \mathrm{~mL}$. In another study more typical of clinical trials in COPD [470], 71 subjects in a crossover trial comparing tiotropium and formoterol had a mean FEV1 of $1.94 \mathrm{~L}$ ( $37 \%$ pred) with an SD of $290 \mathrm{~mL}$. In a study of the effects of tiotropium on exacerbations, pre-treatment FEV1 was $1.60 \mathrm{~L}$ with an SD of $400 \mathrm{~mL}$ (35.6\% pred) [37]. Applying the half SD MID estimate to these clinical therapeutic trials of patients with symptomatic COPD indicates a statistical MID estimate of 145-200 mL.

\section{Professional opinion-based MID}

There are different perspectives on the change in FEV1 felt to be "significant" by professional organisations and clinical guidelines. The ATS/ERS and GOLD suggest that a "significant" response during one test session is a change of $>12 \%$ or $200 \mathrm{~mL}$, whichever is greater [2]. However, this stipulation may simply indicate an improvement that is outside the range observed in normal individuals in response to a short-acting bronchodilator. The ERS has previously suggested that a change of $9 \%$ pred $(\sim 250-300 \mathrm{~mL})$ is a significant response to short-acting bronchodilators [9].

\section{Patient opinion-based MID}

One cross-sectional survey [471] asked 120 patients with COPD to compare their shortness of breath with other subjects enrolled in a pulmonary rehabilitation programme. There was a weak correlation of FEV1 and self-reported dyspnoea $(\mathrm{r}=0.29)$. An FEV1 difference of $4 \%$ (or $112 \mathrm{~mL}$ ) was associated with patients rating their dyspnoea as either slightly better or slightly worse than other patients.

\section{Anchor-based MID}

Although not rigorously evaluated to determine an MID, general results of published investigations in which FEV1 was assessed simultaneously along with other outcomes can provide insight into an FEV1 MID in COPD. Further studies are necessary to refine the FEV1 MID. Data from previously published clinical trials in COPD are likely to provide improved estimates of MID.

One study evaluated the relationship between change in FEV1 and clinical outcomes of acute exacerbations of COPD. In a study by NIEWOEHNER et al. [37] of acute exacerbations of COPD, FEV1 change was associated with clinical response to treatment. An FEV1 improvement of $<100 \mathrm{~mL}$ was associated with a higher relapse rate.

Other studies have shown improvement in FEV1 and effects on exacerbations, but these investigations have not been rigorously analysed to determine a precise MID. In an emergency department study of 147 patients, oral corticosteroid therapy was associated with a mean $140 \mathrm{~mL}$ improvement in FEV1 compared with patients treated with placebo. This change in FEV1 was associated with fewer relapses.

\section{Suggested MID for FEV1}

Table 5 summarises estimates for FEV1 MID using different approaches. As outlined by DoNOHUE [466], further research including additional expert opinion is needed. Because these approaches result in different estimates and there has not been extensive literature addressing the MID for FEV1, an appropriate range of values for the MID for FEV1 might be 100$140 \mathrm{~mL}$.

\section{Other pulmonary function measures}

Due to the cost and complexity of measuring lung volumes, diffusing capacity and arterial blood gases, these features have not been widely employed in research studies of therapeutic outcomes in COPD studies. Therefore, there is limited 
information regarding the MID for pulmonary function measures other than FEV1. However, there has recently been an increased interest in the assessment of hyperinflation and associated measurement of static and dynamic lung volumes in response to bronchodilators and lung volume reduction surgery [49, 58, 224]. Some of these investigations have concurrently measured lung volumes, exercise capacity and quality of life, and could be further analysed to develop external measure-based estimates of MID, particularly for selected lung volume measures. Oxygenation is also a potentially important outcome. Substantial improvements in oxygenation may be associated with reducing or eliminating the need for supplemental oxygen therapy, which is critically important to patients. In addition, reduction in oxygen need may lead to a reduction in healthcare costs, a factor of importance to payers of healthcare and society.

\section{Conclusions}

The MID for outcome measures is a promising method to assist clinicians and investigators in the interpretation of therapeutic trials. However, the MID for some of the outcome measures (pulmonary function) should be more rigorously evaluated according to standard methods before they can be universally applied.

\section{BIOMARKERS}

\section{Introduction}

A biomarker refers to the measurement of any molecule or material (e.g. cells, tissue) that reflects the disease process. In COPD, several types of biomarker have been measured that are related to disease pathophysiology and the inflammatory and destructive process in the lung. Pulmonary biomarkers have been measured in bronchial biopsies, bronchoalveolar lavage (BAL), sputum and exhaled breath. Plasma biomarkers are discussed in the Nonpulmonary markers section. A review of $>600$ published studies suggests that few of these biomarkers have been validated and there is little information about reproducibility and the relationship to disease development, severity or progression [177, 472]. In evaluating pulmonary biomarkers it is important to compare findings in patients with COPD with cigarette smokers matched for exposure who do not have significant airflow limitation (normal smokers) and with age-matched nonsmoking normal subjects. This is rarely performed accurately, making interpretation of abnormal findings difficult. The advantages and

\begin{tabular}{l} 
TABLE $5 \begin{array}{c}\text { Minimal important difference (MID) estimates for } \\
\text { forced expiratory volume in one second (FEV1) }\end{array}$ \\
$\begin{array}{l}\text { Methodology estimates } \\
\text { MID }\end{array}$ \\
$\begin{array}{l}\text { Professional opinion estimate } \\
\%\end{array}$ \\
$\begin{array}{l}\text { mL } \\
\text { Patient opinion estimate }\end{array}$ \\
$\begin{array}{l}\text { mL } \\
\text { Suggested range for FEV1 MID } \\
\text { mL }\end{array}$ \\
\hline
\end{tabular}

disadvantages of various pulmonary biomarkers in COPD have recently been reviewed [473].

\section{Bronchial biopsies}

Although the inflammation in COPD predominately involves lung parenchyma and small airways, bronchial biopsies appear to reflect the cellular abnormalities seen in the peripheral lung $[474,475]$. Bronchial biopsies have been useful for documenting the structural changes, cellular patterns and expression of inflammatory proteins in patients with COPD. In stable COPD there is increased infiltration of macrophages and activated T-lymphocytes, particularly of CD8+ T-lymphocytes $[474,476]$, which express interferon- $\gamma$, inducible protein-10 and interleukin (IL)-9 [477, 478]. Moreover, these lymphocytes express chemokine receptors associated to a type- 1 response, such as CXC chemokine receptor 3, in contrast to lymphocytes in asthma, which express chemokine receptors typical of a type-2 response, such as CC chemokine receptor 4 [479]. While a prominent neutrophilia is present in the airway lumen of patients with COPD in stable conditions, it is not observed at the tissue level, except in patients with severe airflow limitation [480]. Finally, during exacerbations of the disease, an increased recruitment of eosinophils and neutrophils has been described, which is associated with upregulation of specific chemoattractants, such as regulated on activation, normal T-cell expressed and secreted and CXC chemokine ligand 5 [481-483].

Several studies have assessed the potential anti-inflammatory effects of treatments in bronchial biopsies of patients with COPD. These studies usually involve either a baseline biopsy and a second biopsy after a defined period of treatment, or a single biopsy at the end of active treatment with a biopsy in a parallel group of patients taking placebo therapy. Overall, inhaled corticosteroids seem to have little effect on the airway inflammation typical of COPD, while they are able to reduce mast cells, an effect which is associated with a reduction in exacerbation numbers [484, 485]. More encouraging results have been obtained after treatment with either a phosphodiesterase- 4 inhibitor or with the combination of corticosteroids and bronchodilators [486, 487]. However, further studies are required to establish whether the airway inflammation in COPD can be successfully eradicated and whether this would result in a significant clinical improvement.

\section{Advantages}

The main advantage of endobronchial biopsies is that they directly sample airway tissue, maintaining the spatial relationships of structural components that may be important to functional changes [488]. At variance with sputum and BAL, endobronchial biopsies can provide an assessment of structural components of the airway wall, such as epithelium, basement membrane, vessels, connective tissue deposition and, sometimes, smooth muscle and submucosal glands. Therefore, biomarkers of structural damage, such as apoptosis or uncontrolled proliferation, can be measured. Moreover, the different inflammatory cell subtypes can be identified by immunostaining in their microenvironment, thus allowing investigation of interaction between inflammatory and resident cells. Finally, individual structural components can be dissected from the 
biopsies and studied in isolation, using new techniques recently developed, such as laser microdissection [489].

\section{Problems}

There are, however, several limitations to bronchial biopsies as an outcome measurement in COPD. Since this is an invasive procedure, it may be difficult to recruit patients, especially in the studies investigating treatment effects, which require two biopsies (pre- and post-treatment). The biopsy of proximal airways may not closely reflect all the pathological changes present in peripheral airways and lung parenchyma, which are the sites responsible for airflow limitation in COPD. Moreover, it may not be possible to apply this procedure to patients with more severe disease, complicated by cardiac comorbid conditions and often associated with significant oxygen desaturation and hypercapnia [490]. There is also a relatively high variability in baseline measurements of inflammatory cells, which would require multiple biopsies. Finally, since studies evaluating the effect of treatment should be designed to provide a power $\geqslant 80 \%$, a large number of patients for each treatment group is usually required.

\section{Bronchoalveolar lavage}

BAL has the advantage, unlike bronchial biopsies, of sampling inflammation in the lung periphery. BAL can generally be safely performed [490], providing careful assessment is performed and guidelines are adhered to. In general, fluid recovery is greater in patients with less extensive emphysema, as assessed by diffusion capacity [491]. BAL may be performed in the same patients as bronchial biopsy, thus providing additional and complementary information.

\section{Cellular composition}

The cellular composition in individuals with COPD is predominantly $(>80 \%)$ alveolar macrophages, with some neutrophils and T-lymphocytes, and some patients having increased numbers of eosinophils. In general, the percentages of macrophages and neutrophils are significantly higher than in healthy nonsmokers, and also frequently reported in healthy smokers. Studies investigating individuals with COPD, healthy smokers and ex-smokers show that, generally, smoking is associated with increased numbers of neutrophils. Lymphocytes are generally higher in ex-smokers than in smokers, whether with or without COPD. Moreover, some patients with COPD have higher eosinophil percentages than healthy smokers, a finding that is not consistently shown in publications. Alveolar macrophages may be separated by adhesion and cultured in vitro. Macrophages from COPD patients behave abnormally in tissue culture, with increased expression of inflammatory proteins, such as tumour necrosis factor (TNF)- $\alpha$, IL-8 and matrix metalloprotein (MMP)-9 [492, 493]. It may be possible in the future to study the effects of treatment in patients on cellular behaviour in vitro.

\section{Mediators}

Several mediators can be measured in BAL fluid. Levels of eosinophil cationic protein, myeloperoxidase and IL-8 are frequently increased in COPD patients and in healthy smokers compared with healthy nonsmokers, an observation suggesting that smoking induces the changes rather than COPD itself. Two studies investigated tryptase and histamine levels and showed that COPD patients had higher levels as well, suggesting mast cell activation in COPD [494, 495]. However, data were not compared with healthy smokers, and thus the increase in mast cell mediators may be completely attributed to smoking itself. This is also suggested by findings that adenosine monophosphate responsiveness diminishes after smoking cessation [496]. Studies investigating other mediators have not been replicated and are not discussed herein.

\section{Effect of smoking and disease severity}

In one study [497] smokers with COPD had lower mast cell numbers in BAL than ex-smokers with COPD; no other studies have compared smokers and ex-smokers with COPD. Only one study has investigated the association between the severity of COPD and BAL inflammation and shows that healthy smoking males with near-normal FEV1 present signs of inflammation in the lower airways that are related to a decrease in $D \mathrm{~L}, \mathrm{CO}$ and to emphysematous lesions on HRCT [498]. This inflammation seems to be the result of macrophage and neutrophil activation, as assessed by mediators measured in BAL. In contrast, in a healthy population, the number of inflammatory cells did not correlate with lung function decline over a 4 -yr follow-up. However, higher levels of neutrophil elastase- $\alpha_{1}$ protease inhibitor complexes in BAL fluid were significantly correlated with an accelerated decline in FEV1 [499]. This also suggests that the number or percentage of cells is not a prerequisite for the development or progression of emphysema, but that the activation state of these cells with accompanying mediator release is important.

\section{Effects of interventions}

There are few published studies of the effects of different treatments on BAL cellular and mediator components. Three studies, one open label and two double-blind, assessed the effect of different types of inhaled corticosteroid for various periods of treatment on inflammatory cell counts and mediators in BAL. Though the numbers of patients involved were small, precluding firm conclusions, these studies suggest that there may be a reduction in the percentages of neutrophils and lymphocytes with inhaled corticosteroid treatment; however, long-term studies in larger populations must elucidate whether this is indeed the case. Some studies have investigated the effects of smoking cessation on BAL composition, showing inconsistent decreases in cell numbers, particularly macrophages [500, 501].

\section{Problems}

BAL is an invasive procedure and may cause more discomfort to the patient than bronchial biopsy. It may also cause transient fever [490]. The return of fluid is often reduced in COPD patients, resulting in samples that are inadequate for analysis. The quantification of biomarkers in supernatant is a problem as there is no satisfactory marker for the dilution of the saline lavage. This is one of the factors that may contribute to the variability in measurements and the necessity for relatively large numbers of patients.

\section{Sputum}

Many COPD patients produce suitable sputum spontaneously, but spontaneous sputum may contain a high proportion of dead cells [502], which potentially provide misleading cell 
counts and mediator measurements [503, 504]. For this reason, induced sputum has usually been the procedure of choice. It should be recognised that sputum obtained after inhaling nebulised hypertonic saline may have a different composition than mucus and may be more similar to a washing of the proximal airways. The procedure is tolerated by patients with FEV1 $>30 \%$ pred. However, airflow obstruction is often observed [505, 506] and cannot be totally prevented by premedication with $\beta_{2}$-agonists [507].

\section{Inflammatory cells}

There is an abnormal pattern of inflammatory cells in COPD patients, with an increase in number of total inflammatory cells in the percentage of neutrophils and, in some patients, eosinophils (the latter predicting a greater response to corticosteroids) [508, 509]. CD8+ T-cells are increased in induced sputum of COPD patients [510]. Neutrophils have been studied most extensively and are increased in number compared with matched smokers with normal lung function [511]. Several studies have reported the effects of drugs on sputum neutrophils. Most studies have not shown a change in inflammatory cells with inhaled or oral corticosteroids [512514], although a reduction with oral theophylline has been reported [515].

However, it is important to note that, although there is some evidence for the long-term reproducibility of inflammatory cells and mediators [516], the amount of evidence present is still preliminary since the sample sizes investigated are usually too small to extrapolate to the world COPD population.

\section{Inflammatory mediators}

Many mediators have been reported to be increased in the supernatant of COPD patients and most show a greater increase in normal smokers than in COPD, with a further increase during exacerbations; however, few have been related to disease severity or progression. Sputum IL-8 has been studied most extensively and is increased in COPD patients compared with smokers, is related to disease severity (FEV1\% pred) and is further increased with exacerbations [511, 517, 518]. Sputum concentrations are unaffected by corticosteroids but reduced by theophylline [512-514, 519, 520]. Increased proteases have been reported in sputum of patients with COPD, including neutrophil elastase [521] and MMP-8 and -9 [522-524].

\section{Problems}

Although induced sputum samples are relatively easy to obtain in COPD patients and give a lot of information about inflammatory cells and mediators, there are several problems that need to be addressed. Induced sputum samples were obtained from predominantly large airways [525] and may not reflect the peripheral inflammation that may be important for clinical outcomes in COPD. Sputum induction with hypertonic saline induces neutrophilic inflammation that persists for $24 \mathrm{~h}$ and thus repeated sampling within this period is not possible $[525,526]$. Solubilisation of sputum with dithiotheitol (DTT) may disrupt sulphydryl and alter proteins so they are not recognised by antibodies [527]. This is a particular problem with several cytokines and chemokines. Furthermore, proteases in sputum, particularly in COPD, may degrade certain protein mediators. A recent study using dialysis to remove DTT and protease inhibitors [528] showed that it is possible to markedly increase the concentrations of several cytokines in induced sputum of COPD patients. More work is needed on the long-term reproducibility in COPD patients, studying the effect and duration of exacerbations and correlating individual biomarkers with severity and progression.

\section{Exhaled gases}

Measuring biomarkers in the breath is a very attractive approach to monitoring COPD airways inflammation as it is noninvasive and makes repeated sampling possible [529, 530]. However, there are important issues regarding reproducibility and sensitivity that need to be addressed before this approach can be recommended as an outcome measurement.

\section{Nitric oxide}

Exhaled nitric oxide (eNO) has been extensively investigated in asthma and shown to correlate with eosinophilic airway inflammation and to be reduced by corticosteroid therapy. There are ERS and ATS recommendations for measuring exhaled nitric oxide fraction ( $F$ eNO) $[531,532]$. The measurement is highly reproducible in normal and asthmatic subjects if careful attention is paid to technique [533]. However, conventionally measured eNO is less useful in COPD as the levels are usually normal or only slightly elevated, except during exacerbations [534-538]. This is likely to be due to the increase in oxidative stress, resulting in the formation of peroxynitrite and nitrate, so that nitric oxide (NO) is removed from the gaseous phase. This also explains why eNO is reduced in normal smokers [539].

Recently, the measurement of eNO has been extended by performing measurements of eNO at different flows, so that it is possible to partition airway-derived $\mathrm{NO}$, which is flowindependent, and peripheral NO derived from alveoli and probably the small airways. Using this technique it is possible to show that, while airway NO is low or normal on COPD, there is an increase in peripheral NO that is related to disease severity [540]. This may reflect the increase in inducible NO synthase in the lung periphery of patients with COPD [541]. This peripheral NO may prove to be a useful noninvasive biomarker of COPD inflammation but further studies on reproducibility relationship to disease severity and the effects of treatments are now needed.

\section{Carbon monoxide}

Although it is easy to measure $\mathrm{CO}$ in the breath, this has not proven to be as useful a measurement as FeNO. Exhaled $\mathrm{CO}$ is elevated in patients with COPD but it is also elevated in normal smokers due to the high $\mathrm{CO}$ content in cigarette smoke $[538,542]$. Exhaled CO is elevated to a greater extent in COPD than in matched normal smokers and remains elevated in sustained ex-smokers. However, the signal is small and the measurement is also confounded by highly variable environmental CO levels and the effects of passive smoking, so further evaluation is not warranted.

\section{Hydrocarbons}

Volatile hydrocarbons, such as ethane and pentane, have been detected in exhaled breath and are biomarkers of lipid peroxidation as a result of oxidative stress. Concentrations of 


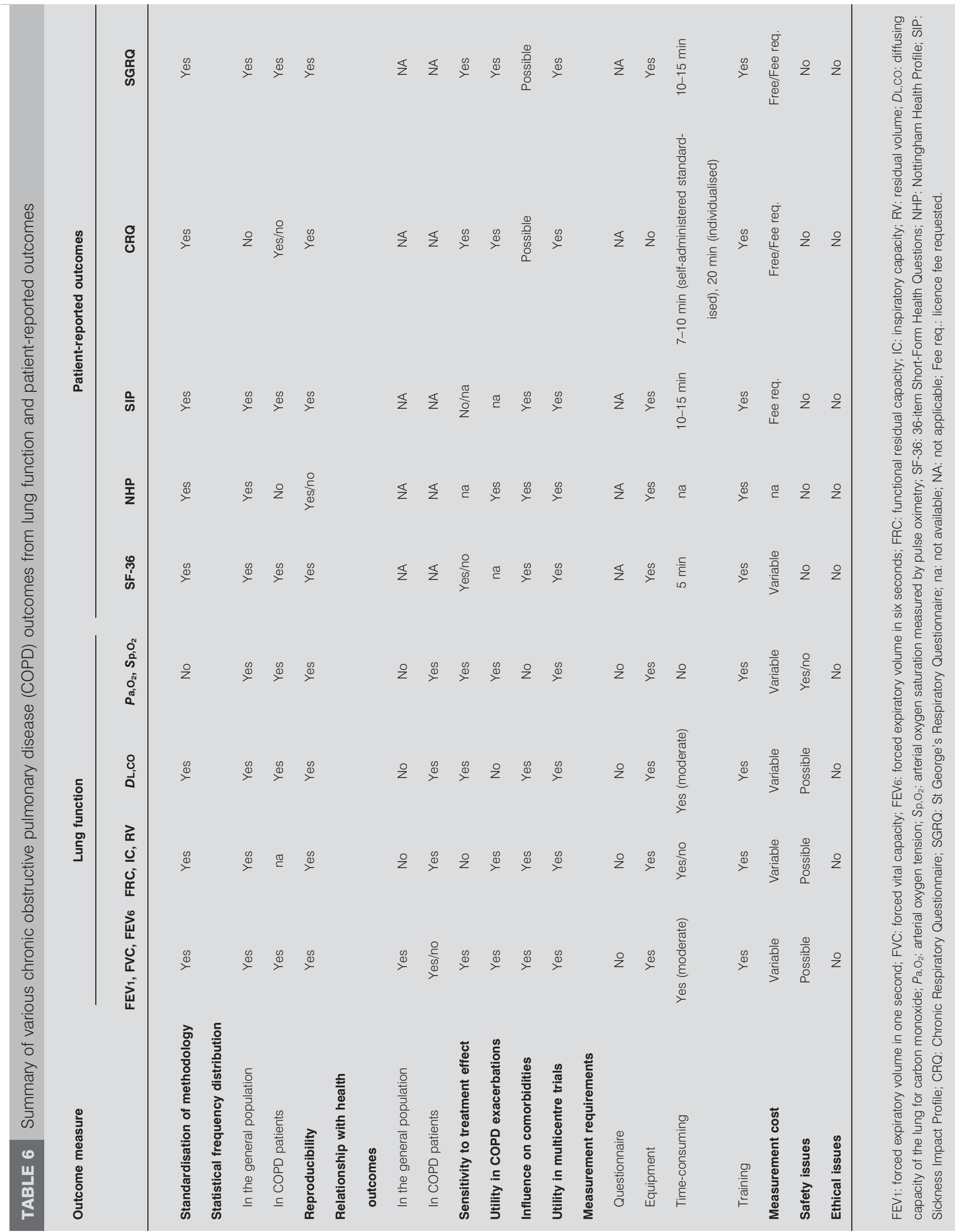


TABLE 7 Summary of various chronic obstructive pulmonary disease (COPD) outcomes from patient-reported outcomes

\begin{tabular}{|c|c|c|c|c|c|c|c|c|c|c|}
\hline \multirow[t]{2}{*}{ Outcome measure } & \multicolumn{10}{|c|}{ Patient-reported outcomes } \\
\hline & QoL-RIQ & AQ20 & $\mathrm{BPQ}$ & MRF-28 & $\operatorname{CCQ}$ & PFSDQ-M & PFSS & EADL & LCADL & QWB \\
\hline $\begin{array}{l}\text { Standardisation of } \\
\text { methodology }\end{array}$ & Yes & Yes & Yes & Yes & Yes & Yes & Yes & Yes & Yes & Yes \\
\hline \multicolumn{11}{|l|}{$\begin{array}{l}\text { Statistical frequency } \\
\text { distribution }\end{array}$} \\
\hline In COPD patients & na & Yes & Yes & Yes & Yes & na & na & na & na & na \\
\hline Reproducibility & na & na & Yes & na & na & Yes & Yes & na & Yes & Yes \\
\hline \multicolumn{11}{|l|}{$\begin{array}{l}\text { Relationship with health } \\
\text { outcomes }\end{array}$} \\
\hline In the general population & NA & NA & NA & NA & NA & NA & NA & NA & NA & NA \\
\hline In COPD patients & NA & NA & NA & NA & NA & NA & NA & NA & NA & NA \\
\hline Influence on comorbidities & Possible & Possible & Possible & Possible & Possible & Possible & Possible & Yes & Possible & Yes \\
\hline Utility in multicentre trials & Possible & Possible & Possible & Possible & Possible & Yes & na & Yes & Yes & Yes \\
\hline \multicolumn{11}{|l|}{ Measurement requirements } \\
\hline Questionnaire & Yes & Yes & Yes & Yes & Yes & Yes & Yes & Yes & Yes & Yes \\
\hline Equipment & NA & NA & NA & NA & NA & NA & NA & na & na & Yes \\
\hline Time-consuming & na & $3 \mathrm{~min}$ & na & na & $2 \mathrm{~min}$ & 15-20 min & $15 \mathrm{~min}$ & $5-10 \mathrm{~min}$ & na & 10-20 min \\
\hline Training & Yes & Yes & Yes & Yes & Yes & Yes & Yes & Yes & Yes & Yes \\
\hline Measurement cost & na & Free & Free & na & na & na & na & na & na & Fee req. \\
\hline Safety issues & No & No & No & No & No & No & No & No & No & No \\
\hline Ethical issues & No & No & No & No & No & No & No & No & No & No \\
\hline
\end{tabular}

QoL-RIQ: Quality of Life For Respiratory IIIness Questionnaire; AQ20: Airways Questionnaire 20; BPQ: Breathing Problems Questionnaire; MRF-28: Maugeri Foundation Respiratory Failure Questionnaire; CCQ: Clinical Control Questionnaire; PFSDQ-M: Pulmonary Functional Status and Dyspnoea Questionnaire; PFSS: Pulmonary Functional Status Scale; EADL: Nottingham Extended Activity of Daily Living Scale; LCADL: London Chest Activity of Daily Living Scale; QWB: Quality of Well-Being Scale; na: not available; NA: not applicable; Fee req.: licence fee requested.

ethane are elevated in patients with COPD and correlated with disease severity [542]. Measurement of ethane by gas chromatography-mass spectrometry offline is difficult, so this measurement is unlikely to be useful in clinical trials, but smaller and more sensitive detectors for hydrocarbons are now in development.

\section{Exhaled breath condensate}

Many mediators have now been detected in exhaled breath condensate (EBC), which has the advantage that it is easy to perform and completely noninvasive [543]. Several factors affect the measurement and recommendations have recently been formulated by an ERS/ATS Task Force [544]. A limitation of the technique is the variability of the measurement and the low concentrations of mediators detected.

\section{Oxidative/nitrative stress}

Hydrogen peroxide $\left(\mathrm{H}_{2} \mathrm{O}_{2}\right)$ is increased in EBC of COPD patients, is further increased during exacerbations [545] and is related to disease severity [546]. Exhaled $\mathrm{H}_{2} \mathrm{O}_{2}$ is reported to be reproducible in repeated measurements over 3 days [547].
8-Isoprostane is a stable marker of oxidative stress and is also increased in EBC of COPD patients. Concentrations of 8isoprostane are greater in COPD patients than normal smokers and are related to disease severity [548-550], and further increased during exacerbations [551]. Certain aldehydes resulting from lipid peroxidation are also increased in COPD patients but only malondialdehyde is increased in COPD patients compared with normal smokers [552]. Increased nitrosative stress in COPD is indicated by increased concentration of nitrite and nitrosothiols in EBC [553].

\section{Inflammatory mediators}

Inflammation is associated with tissue acidification and there is a decrease in $\mathrm{pH}$ in EBC of COPD patients [554]. There is considerable variability in exhaled $\mathrm{pH}$ in COPD patients, which is greater than in normal subjects [555]. There is an increase in the concentration of leukotriene $B_{4}$ in COPD patients, which is further increased during exacerbations [551, 556, 557]. Increases in prostaglandin $\mathrm{E}_{2}$ and IL-6 have also been reported in COPD patients [556, 558]. It is unclear how most of these biomarkers relate to disease 


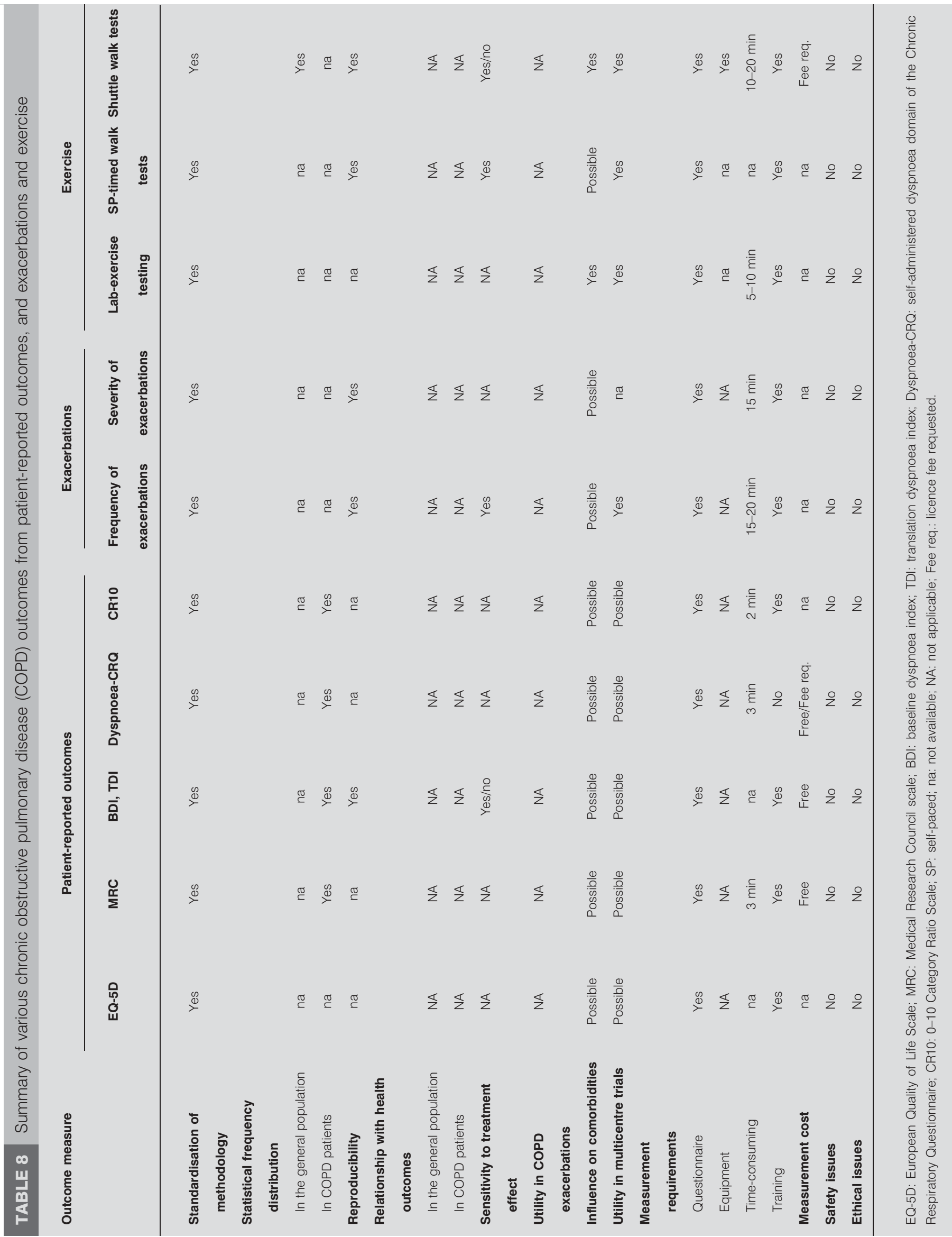




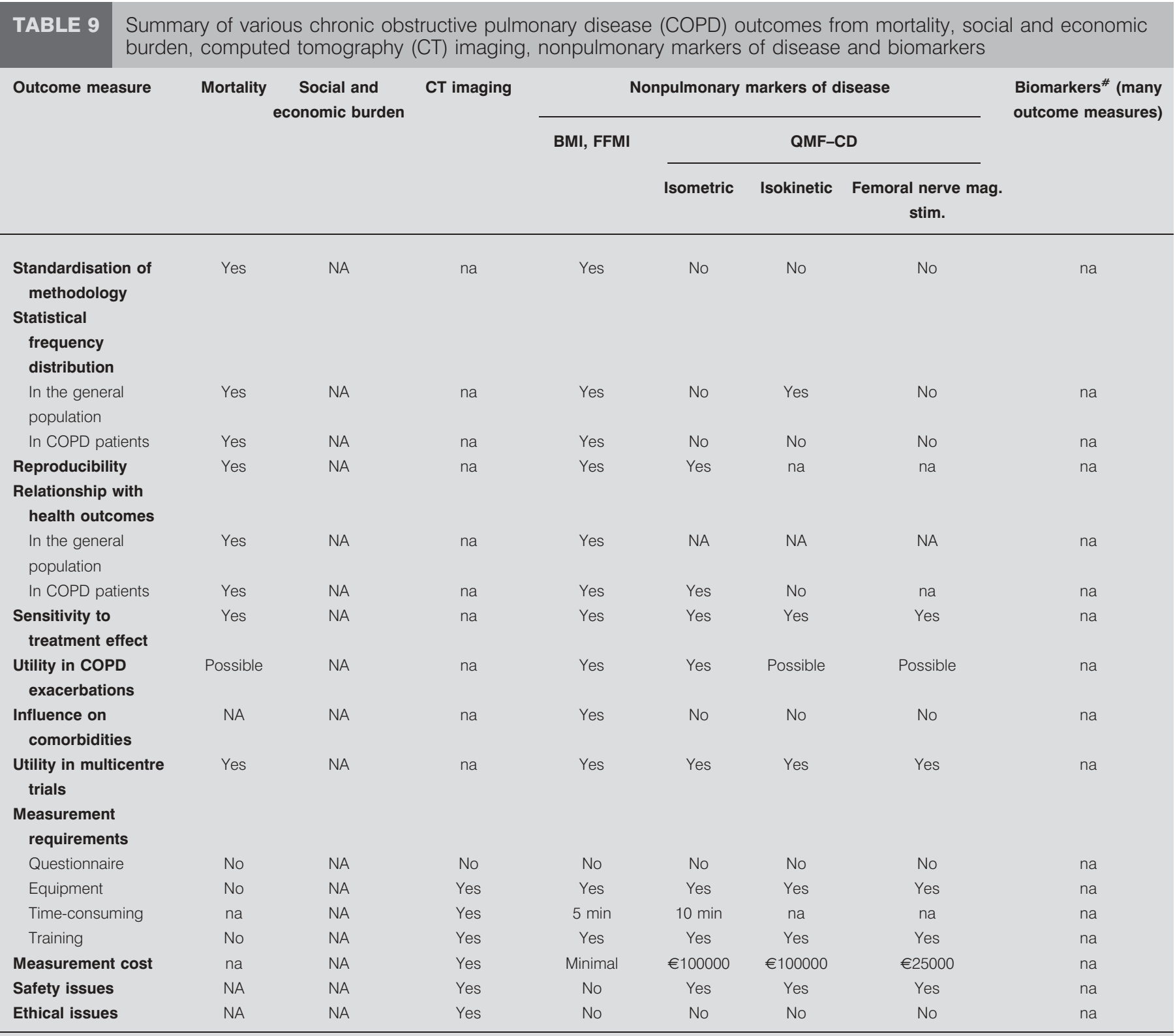

BMI: body mass index; FFMI: fat-free mass index; QMF: quadriceps muscle function; CD: computerised dynamometer; mag. stim.: magnetic stimulation; na: not available; NA: not applicable. ${ }^{\#}$ : there are many suggested biomarkers in the medical literature; however, they are often obtained from studies with small sample sizes and/or not replicated by other independent research groups.

severity and patient-centred outcomes. Most protein mediators, including cytokines and enzymes, cannot reliably be measured in EBC.

\section{Problems}

There is a relative high variability in repeated measurements of EBC biomarkers and this may relate to the extensive variable dilution that occurs from water vapour during condensation and the low concentrations that may be near to the detection limits of the assays used [559]. Further work is needed to optimise these measurements and to determine the causes of variability. Correction for the variable dilution is one approach [560]. Assays are usually performed using ELISA and these assays have been validated using gas chromatography-mass spectrometry for some mediators [561, 562].

\section{Blood}

In recent years, researchers have hypothesised that a "lowgrade" systemic inflammation may play an important role in the pathogenesis of systemic complications observed in chronic respiratory diseases such as COPD [248, 252, 268, 563-568]. In the Third National Health and Nutrition Examination Survey of the US population [564], a number of markers of systemic inflammation were shown to be associated with active smoking and reduced FEV1. The markers investigated were C-reactive protein (CRP), fibrinogen, leukocytes 
and platelets in serum and plasma. However, their level of involvement has since been questioned due to the crosssectional methodology used and the lack of information regarding the temporal aspects and biological plausibility of this observed association [569]. Previous studies [566, 568, 570584] have suggested roles for systemic CRP, fibrinogen, leukocytes, TNF- $\alpha$, IL- 6 and IL- 8 in COPD and its exacerbations, but more longitudinal randomised controlled studies with larger sample sizes are needed to confirm their specificity and sensitivity as biomarkers in patients with reduced lung function [441].

\section{Regulatory issues}

Many drugs are now in development as potential antiinflammatory therapies for COPD [585]. However, as there is no effective anti-inflammatory treatment for COPD, it is uncertain how much and how rapidly clinical parameters will change in patients. This makes it important to develop reliable biomarkers to quantify inflammation in COPD patients and to validate these against some other measure of disease activity and progression. For assessment of anti-inflammatory treatments it is important to identify biomarkers that indicate the efficacy of the drug on components of the inflammatory process before proceeding to large and prolonged clinical trials. Biomarkers can facilitate drug development in a number of ways, such as: 1) providing evidence that a drug can reach its target and modify that target in some positive way; 2) identifying criteria for dose selection for phase- 2 and -3 studies; 3) providing "go-no-go" decisions at early stages of the drugdevelopment process; 4) identifying populations that are more likely to benefit from a drug; and 5) predicting safety problems.

There are several types of drugs that can be developed for COPD based on whether the drug is intended to improve airflow obstruction, provide symptom relief, modify or prevent exacerbations, alter disease progression, or modify lung structure. The efficacy end-points that are currently used in phase-3 studies to support registration of a drug for COPD are based on measures that translate to direct benefit of some aspects of the disease that is important to patients, such as improvement of symptoms, functional capacity, HRQoL or survival. With the possible exception of drugs that are intended to improve airflow obstruction, whose efficacy can be relatively easily assessed by measuring FEV1 in short-term studies, drugs of other types are likely to require prolonged studies, often extending to many years. These studies become rather risky and expensive endeavours and this further underscores the need for the development of biomarkers.

The biomarkers described elsewhere in the present report are not sufficiently validated to date for use as evidence of efficacy in phase-3 studies or for supporting specific labelling claims. Nevertheless, these biomarkers are reflective of the disease and have potential use for regulatory purpose. Carefully selected biomarkers with or without a patient-centred clinically meaningful end-point can be used in early phase studies, such as proof-of-action or proof-of-concept studies, based on which a rational decision can be made on further development of the drug. Biomarkers can also be used in either early phase studies or phase-3 studies to support the drug's putative mode of action. In addition, use of the biomarkers in phase- 3 studies in conjunction with clinically meaningful end-points may help validate the use of the biomarker, or even help elevate a biomarker to a surrogate end-point status.

\section{Conclusions}

Although many pulmonary biomarkers have been described in COPD patients, there is little information regarding reproducibility and correlation with other outcome measurements in COPD (i.e. dyspnoea, HRQoL, exacerbation frequency and mortality). In the future, these biomarkers need to be assessed in normal smokers and age-matched normal subjects and linked to disease stage (and rate of FEV1 decline), clinical phenotype (emphysema versus small airway disease), smoking status (current versus ex-smokers), clinical status (stable versus exacerbation) and treatment (effect of corticosteroids, theophylline, etc.). Further research in this area is important as pulmonary biomarkers may be useful in the future for predicting clinical outcomes of COPD and for assessing new therapies which may modify the inflammatory/destructive disease process.

\section{SUMMARY AND CONCLUSIONS}

By the time patients with COPD seek medical attention, they usually have significant symptoms, especially dyspnoea, reduced exercise performance and impaired health status. These aspects of COPD morbidity have been investigated for many years and their association with the disease process has resulted in measurable outcomes used for the assessment of pharmacological treatment. The ATS/ERS Task Force has summarised many of these outcomes in tables 6-9. Although some of these outcomes have been shown to change with therapy, their observed changes are not always reflected by changes in traditional measures of disease severity such as FEV1. This is because other pathophysiological (e.g. dynamic hyperinflation of the lungs) and psychological (e.g. coexisting anxiety) influences also affect these outcomes. Therefore, changes in FEV1 with therapy should not be regarded as a surrogate for changes in dyspnoea, exercise performance or HRQoL. These variables should be measured separately to complement other markers of physiological impairment when assessing a therapy for COPD [6].

Therefore, it is necessary that COPD trials include lung function parameters other than FEV1; for example, FVC and IC to TLC ratio, measures of dyspnoea, functional status, health status and HRQoL, exercise tolerance, and breathlessness after exercise.

The frequency of exacerbations is another important outcome that should be considered in COPD pharmacological trials. The definition used for an exacerbation can significantly affect trial outcomes to the extent that any observed treatment benefit may vary. A general definition, such as "an exacerbation of COPD is an increase in respiratory symptoms over baseline that usually requires medical intervention", may be more applicable. However, exacerbations should be classified according to a severity scale.

Currently, no well-validated biomarker or surrogate marker of COPD or its exacerbations has been identified other than FEV1, but the value of FEV1 as a surrogate marker is limited. Mortality, dyspnoea and HRQoL remain the most important and robust clinical outcomes in COPD research. Care should 
be taken to include other potential surrogate markers as secondary end-points in future clinical trials. This may lead to the identification of biomarkers that correlate with patientcentred outcomes. Generation of such data may also help in the development of new hypotheses for future clinical trials [3].

Based on the rate of disease progression and the frequency of exacerbations, it is now recognised that pharmacological trials in stable chronic obstructive pulmonary disease should be $\geqslant 6$ months in order to examine potential outcomes or support claims of treatment response, particularly for regulatory submissions. However, due to seasonal variation, an evaluation of exacerbation frequency requires a period of $\geqslant 1 \mathrm{yr}$ and, in any case, the timing of the study treatment may prove important (e.g. capturing winter cold season in the majority of patients).

A minimal important difference between treated and untreated groups in small studies using interventions of limited efficacy may not be observed, bearing in mind the slow progression of chronic obstructive pulmonary disease (as measured by forced expiratory volume in one second). However, those who design clinical trials should be aware that the comparison of proportions of patients reaching the minimal important difference may provide important information, even if the mean effect does not exceed the minimal important difference. In general, a randomised controlled trial design is the most useful study design for determining the effect of treatment on outcomes, including the rate of forced expiratory volume in one second loss and the change in frequency of chronic obstructive pulmonary disease exacerbations. Only placebocontrolled trials enable analysis of the effect of active treatment, but control groups should always receive the best available proven treatment and, consequently, use of placebo raises ethical issues.

\section{ACKNOWLEDGEMENTS}

Affiliations were as follows. From Italy: M. Cazzola, University of Rome Tor Vergata and P. Palange, University of Rome La Sapienza, Rome; V. Brusasco, University of Genoa, Genoa; and M. Pistolesi, University of Florence, Florence. From the UK: W. MacNee, Colt Research Laboratories, Medical School, Edinburgh; L.G. Franciosi, King's College London, P.J. Barnes, National Heart and Lung Institute, Imperial College London, P.W. Jones, St George's Hospital, University of London, C.P. Page, Sackler Institute of Pulmonary Pharmacology, King's College Hospital Medical School and J.A. Wedzicha, University College London, London; P.S. Burge, Birmingham Heartlands Hospital and R. Stockley, Queen Elizabeth Hospital, Birmingham; D. Parr, University Hospitals of Coventry and Warwickshire, Coventry; and P.M.A. Calverley, University Hospital Aintree, Liverpool. From the USA: F.J. Martinez, University of Michigan Medical School, Ann Arbor, MI; B.R. Celli, Caritas-St. Elizabeth's Medical Center, Boston, MA; D.A. Mahler, DartmouthHitchcock Medical Center, Lebanon, NH; B. Make, National Jewish Medical and Research Center, Denver, CO; and S.D. Sullivan, University of Washington, Seattle, WA. From the Netherlands: K.F. Rabe, Leiden University Medical Center, Leiden; M.P. Rutten-van Mölken, Erasmus MC-University Medical Centre Rotterdam, Rotterdam; and E.F. Wouters, University Hospital Maastricht, Maastricht. From Spain: M. Miratvilles, Hospital Clínic, Barcelona.
Participants in the Task Force report were as follows.

From the UK: I.M. Adcock (Imperial College School of Medicine, London), N. Barnes (London Chest Hospital, London), P.J. Barnes (National Heart and Lung Institute, Imperial College London, London), P.S. Burge (Birmingham Heartlands Hospital, Birmingham), P.M.A. Calverley (University Hospital Aintree, Liverpool), R. Djukanovic (University of Southampton, Southampton), P.W. Jones (St George's Hospital, University of London, London), S. Kharitonov (National Heart and Lung Institute, Imperial College London, London), W. MacNee (Colt Research Laboratories, Medical School, Edinburgh), D. Parr (University Hospitals of Coventry and Warwickshire, Coventry), R. Stockley (Queen Elizabeth Hospital, Edgbaston, Birmingham), J. Vestbo (University of Manchester, Manchester) and J.A. Wedzicha (University College London, London).

From Sweden: L. Bjermer (University Hospital, Lund) and C.G. Löfdahl (Lund University, Lund).

From Italy: V. Brusasco (University of Genoa, Genoa), M. Cazzola (University of Rome Tor Vergata, Rome), G. D'Amato (A. Cardarelli Hospital, Naples), C.F. Donner (Multidisciplinary and Rehabilitation Outpatient Clinic, Borgomanero), L.M. Fabbri (University of Modena, Modena), P.L. Paggiaro (University of Pisa, Pisa), P. Palange (University of Rome La Sapienza, Rome), M. Pistolesi (University of Florence, Florence), A. Rossi (Bergamo General Hospital, Bergamo), M. Saetta (University of Padua, Padua), G. Viegi (CNR Institute of Clinical Physiology, Pisa) and A.M. Vignola ${ }^{\dagger}$ (Institute of Lung Pathophysiology, National Research Council, Palermo).

From Germany: R. Buhl (University Hospital, Mainz) and H. Magnussen (Center for Pneumology and Thoracic Surgery, Großhansdorf).

From the USA: S. Buist (Portland Oregon Health and Science University, Portland, OR), B.R. Celli (Caritas-St Elizabeth's Medical Center, Boston, MA), D.A. Mahler (DartmouthHitchcock Medical Center, Lebanon, NH), B. Make (National Jewish Medical and Research Center, Denver, CO), F.J. Martinez (University of Michigan Medical School, Ann Arbor, MI), S.I. Rennard (University of Nebraska Medical Center, Omaha, NE), B. Chowdhury (Center for Drug Evaluation and Research, Food and Drug Administration, Silver Spring, MD) and S.D. Sullivan (University of Washington, Seattle, WA).

From Denmark: R. Dahl (Aarhus University Hospital, Aarhus). From Japan: M. Ichinose (Wakayama University Hospital, Wakayama).

From the Netherlands: D. Postma (University Medical Center Groningen, Groningen), K. F. Rabe (Leiden University Medical Center, Leiden), M.P. Rutten-van Mölken (Institute for Medical Technology Assessment, Rotterdam) and E.F. Wouters (University Hospital Maastricht, Maastricht).

From Belgium: G. Joos and R. Pauwels ${ }^{\dagger}$ (Ghent University Hospital, Ghent). 
From Spain: M. Miravitlles (Hospital Clínic, Barcelona) and J. Roca (Hospital Clínic, IDIBAPS, Universitat de Barcelona, Barcelona).

From Argentina: L. Nannini (G. Baigorria Hospital, National University of Rosario, Rosario).

From Ireland: D. Lyons (Irish Medicines Board, Dublin).

\section{REFERENCES}

1 Committee for Proprietary Medicinal Products. Points to consider on clinical investigation of medicinal products in the chronic treatment of patients with chronic obstructive pulmonary disease (COPD). London, CPMP/EWP/562/98, 1999.

2 Celli BR, MacNee W. Standards for the diagnosis and treatment of patients with COPD: a summary of the ATS/ERS position paper. Eur Respir J 2004; 23: 932-946.

3 Edwards N. A framework for future trials: Guidelines on Developing COPD Drugs. GCPJ 2004; 11: 1-5.

4 Jones PW, Agusti AGN. Outcomes and markers in the assessment of chronic obstructive pulmonary disease. Eur Respir J 2006; 27: 822-832.

5 Celli BR, Calverley PM, Rennard SI, et al. Proposal for a multidimensional staging system for chronic obstructive pulmonary disease. Respir Med 2005; 99: 1546-1554.

6 ZuWallack RL, Haggerty MC, Jones P. Clinically meaningful outcomes in patients with chronic obstructive pulmonary disease. Am J Med 2004; 117: Suppl. 12A, 49S-59S.

7 Miller MR, Hankinson J, Brusasco V, et al. Standardisation of spirometry. Eur Respir J 2005; 26: 319-338.

8 Lung function testing: selection of reference values and interpretative strategies. American Thoracic Society. Am Rev Respir Dis 1991; 144: 1202-1218.

9 Quanjer PH, Tammeling GJ, Cotes JE, Pedersen OF, Peslin R, Yernault JC. Lung volumes and forced ventilatory flows. Report Working Party Standardization of Lung Function Tests, European Community for Steel and Coal. Official Statement of the European Respiratory Society. Eur Respir J 1993; 16: Suppl. 16, 5-40.

10 Pellegrino R, Viegi G, Brusasco V, et al. Interpretative strategies for lung function tests. Eur Respir J 2005; 26: 948-968.

11 Global Initiative for Chronic Obstructive Lung Disease. Global strategy for the diagnosis, management and prevention of chronic obstructive pulmonary disease. NHLBI/WHO Workshop Report 2005. www.goldcopd. com Date last accessed: November 2, 2007.

12 Anthonisen NR, Connett JE, Kiley JP, et al. Effects of smoking intervention and the use of an inhaled anticholinergic bronchodilator on the rate of decline of FEV1. The Lung Health Study. JAMA 1994; 272: 1497-1505.

13 Anonymous. GSK's Ariflo gets US approvable letter for COPD. Scrip World Pharmaceutical News October 31, 2003; 21: No. 2898.

14 Swanney MP, Jensen RL, Crichton DA, Beckert LE, Cardno LA, Crapo RO. FEV6 is an acceptable surrogate for FVC in the spirometric diagnosis of airway obstruction and restriction. Am J Respir Crit Care Med 2000; 162: $917-$ 919.

15 Hankinson JL, Crapo RO, Jensen RL. Spirometric reference values for the 6-s FVC maneuver. Chest 2003, 124: 1805-1811.

16 Garcia-Rio F, Pino JM, Dorgham A, Alonso A, Villamor J. Spirometric reference equations for European females and males aged 65-85 yrs. Eur Respir J 2004; 24: 397-405.

17 Vandevoorde J, Verbanck S, Schuermans D, Kartounian J, Vincken W. FEV1/FEV6 and FEV6 as an alternative for FEV1/FVC and FVC in the spirometric detection of airway obstruction and restriction. Chest 2005; 127: 1560-1564.

18 Akpinar-Elci M, Fedan KB, Enright PL. FEV6 as a surrogate for FVC in detecting airways obstruction and restriction in the workplace. Eur Respir J 2006; 27: 374-377.

19 Pedersen OF. FEV6: a shortcut in spirometry? Eur Respir J 2006; 27: 245-247.

20 Vandevoorde J, Verbanck S, Schuermans D, Kartounian J, Vincken W. Obstructive and restrictive spirometric patterns: fixed cut-offs for FEV1/FEV6 and FEV6. Eur Respir J 2006; 27: 378-383.

21 Bang KM, Gergen PJ, Kramer R, Cohen B. The effect of pulmonary impairment on all-cause mortality in a national cohort. Chest 1993; 103: 536-540.

22 Neas LM, Schwartz J. Pulmonary function levels as predictors of mortality in a national sample of US adults. Am J Epidemiol 1998; 147: 1011-1018.

23 Knuiman MW, James AL, Divitini ML, Ryan G, Bartholomew HC, Musk AW. Lung function, respiratory symptoms, and mortality: results from the Busselton Health Study. Ann Epidemiol 1999; 9: 297-306.

24 Schunemann HJ, Dorn J, Grant BJ, Winkelstein W Jr, Trevisan M. Pulmonary function is a long-term predictor of mortality in the general population: 29-year follow-up of the Buffalo Health Study. Chest 2000; 118: 656-664.

25 Stavem K, Aaser E, Sandvik L, et al. Lung function, smoking and mortality in a 26-year follow-up of healthy middle-aged males. Eur Respir J 2005; 25: 618-625.

26 Wolkove N, Dajczman E, Colacone A, Kreisman H. The relationship between pulmonary function and dyspnea in obstructive lung disease. Chest 1989; 96: 1247-1251.

27 Hay JG, Stone P, Carter J, et al. Bronchodilator reversibility, exercise performance and breathlessness in stable chronic obstructive pulmonary disease. Eur Respir J 1992; 5: 659-664.

28 Bauerle O, Chrusch CA, Younes M. Mechanisms by which COPD affects exercise tolerance. Am J Respir Crit Care Med 1998; 157: 57-68.

29 O'Donnell DE, Lam M, Webb KA. Spirometric correlates of improvement in exercise performance after anticholinergic therapy in chronic obstructive pulmonary disease. Am J Respir Crit Care Med 1999; 160: 542-549.

30 Ferrer M, Alonso J, Prieto L, et al. Validity and reliability of the St George's Respiratory Questionnaire after adaptation to a different language and culture: the Spanish example. Eur Respir J 1996; 9: 1160-1166.

31 Hajiro T, Nishimura K, Tsukino M, Ikeda A, Koyama H, Izumi T. Comparison of discriminative properties among disease-specific questionnaires for measuring healthrelated quality of life in patients with chronic obstructive 
pulmonary disease. Am J Respir Crit Care Med 1998; 157: 785-790.

32 Pellegrino R, Rodarte JR, Brusasco V. Assessing the reversibility of airway obstruction. Chest 1998; 114: 1607-1612.

33 Calverley PM, Burge PS, Spencer S, Anderson JA, Jones PW. Bronchodilator reversibility testing in chronic obstructive pulmonary disease. Thorax 2003; 58: 659-664.

34 The National Emphysema Treatment Trial Research Group. Rationale and design of The National Emphysema Treatment Trial: a prospective randomized trial of lung volume reduction surgery. Chest 1999; 116: 1750-1761.

35 Seemungal TA, Donaldson GC, Bhowmik A, Jeffries DJ, Wedzicha JA. Time course and recovery of exacerbations in patients with chronic obstructive pulmonary disease. Am J Respir Crit Care Med 2000; 161: 1608-1613.

36 Aaron SD, Vandemheen KL, Clinch JJ, et al. Measurement of short-term changes in dyspnea and disease-specific quality of life following an acute COPD exacerbation. Chest 2002; 121: 688-696.

37 Niewoehner DE, Collins D, Erbland ML. Relation of FEV1 to clinical outcomes during exacerbations of chronic obstructive pulmonary disease. Department of Veterans Affairs Cooperative Study Group. Am J Respir Crit Care Med 2000; 161: 1201-1205.

38 Parker CM, Voduc N, Aaron SD, Webb KA, O'Donnell DE. Physiological changes during symptom recovery from moderate exacerbations of COPD. Eur Respir J 2005; 26: 420-428.

39 Cerveri I, Pellegrino R, Dore R, et al. Mechanisms for isolated volume response to a bronchodilator in patients with COPD. J Appl Physiol 2000; 88: 1989-1995.

40 van Schayck CP, Loozen JM, Wagena E, Akkermans RP, Wesseling GJ. Detecting patients at a high risk of developing chronic obstructive pulmonary disease in general practice: cross sectional case finding study. BMJ 2002; 324: 1370.

41 Miller MR, Crapo R, Hankinson J, et al. General considerations for lung function testing. Eur Respir J 2005; 26: 153-161.

42 Wanger J, Clausen JL, Coates A, et al. Standardisation of the measurement of lung volumes. Eur Respir J 2005; 26: 511-522.

43 Stocks J, Quanjer PH. Reference values for residual volume, functional residual capacity and total lung capacity. ATS Workshop on Lung Volume Measurements. Official Statement of The European Respiratory Society. Eur Respir J 1995; 8: 492-506.

44 Hankinson JL, Stocks J, Peslin R. Reproducibility of lung volume measurements. Eur Respir J 1998; 11: 787-790.

45 O'Donnell DE, Revill SM, Webb KA. Dynamic hyperinflation and exercise intolerance in chronic obstructive pulmonary disease. Am J Respir Crit Care Med 2001; 164: 770-777.

46 Boni E, Corda L, Franchini D, et al. Volume effect and exertional dyspnoea after bronchodilator in patients with COPD with and without expiratory flow limitation at rest. Thorax 2002; 57: 528-532.

47 O'Donnell DE, Webb KA. Exertional breathlessness in patients with chronic airflow limitation. The role of lung hyperinflation. Am Rev Respir Dis 1993; 148: 1351-1357.
48 O’Donnell DE, Webb KA, Bertley JC, Chau LK, Conlan AA. Mechanisms of relief of exertional breathlessness following unilateral bullectomy and lung volume reduction surgery in emphysema. Chest 1996; 110: 18-27.

49 O'Donnell DE, Fluge T, Gerken F, et al. Effects of tiotropium on lung hyperinflation, dyspnoea and exercise tolerance in COPD. Eur Respir J 2004; 23: 832-840.

50 O'Donnell DE, Voduc N, Fitzpatrick M, Webb KA. Effect of salmeterol on the ventilatory response to exercise in chronic obstructive pulmonary disease. Eur Respir J 2004; 24: 86-94.

51 Diaz O, Villafranca C, Ghezzo H, et al. Role of inspiratory capacity on exercise tolerance in COPD patients with and without tidal expiratory flow limitation at rest. Eur Respir J 2000; 16: 269-275.

52 Marin JM, Carrizo SJ, Gascon M, Sanchez A, Gallego B, Celli BR. Inspiratory capacity, dynamic hyperinflation, breathlessness, and exercise performance during the 6-minute-walk test in chronic obstructive pulmonary disease. Am J Respir Crit Care Med 2001; 163: 1395-1399.

53 Newton MF, O’Donnell DE, Forkert L. Response of lung volumes to inhaled salbutamol in a large population of patients with severe hyperinflation. Chest 2002; 121: 1042-1050.

54 Murciano D, Ferretti A, Boczkowski J, Sleiman C, Fournier M, Milic-Emili J. Flow limitation and dynamic hyperinflation during exercise in COPD patients after single lung transplantation. Chest 2000; 118: 1248-1254.

55 Casanova C, Cote C, de Torres JP, et al. Inspiratory-tototal lung capacity ratio predicts mortality in patients with chronic obstructive pulmonary disease. Am J Respir Crit Care Med 2005; 171: 591-597.

56 Ingenito EP, Loring SH, Moy ML, Mentzer SJ, Swanson SJ, Reilly JJ. Physiological characterization of variability in response to lung volume reduction surgery. J Appl Physiol 2003; 94: 20-30.

57 Tantucci C, Duguet A, Similowski T, Zelter M, Derenne JP, Milic-Emili J. Effect of salbutamol on dynamic hyperinflation in chronic obstructive pulmonary disease patients. Eur Respir J 1998; 12: 799-804.

58 Maltais F, Hamilton A, Marciniuk D, et al. Improvements in symptom-limited exercise performance over $8 \mathrm{~h}$ with once-daily tiotropium in patients with COPD. Chest 2005; 128: $1168-1178$.

59 O'Donnell DE. Is sustained pharmacologic lung volume reduction now possible in COPD? Chest 2006; 129: 501-503.

60 Celli B, ZuWallack R, Wang S, Kesten S. Improvement in resting inspiratory capacity and hyperinflation with tiotropium in COPD patients with increased static lung volumes. Chest 2003; 124: 1743-1748.

61 van Noord JA, Aumann JL, Janssens E, et al. Effects of tiotropium with and without formoterol on airflow obstruction and resting hyperinflation in patients with COPD. Chest 2006; 129: 509-517.

62 Stevenson NJ, Walker PP, Costello RW, Calverley PM. Lung mechanics and dyspnea during exacerbations of chronic obstructive pulmonary disease. Am J Respir Crit Care Med 2005; 172: 1510-1516.

63 Duranti R, Filippelli M, Bianchi R, et al. Inspiratory capacity and decrease in lung hyperinflation with albuterol in COPD. Chest 2002; 122: 2009-2014. 
64 Dolmage TE, Goldstein RS. Repeatability of inspiratory capacity during incremental exercise in patients with severe COPD. Chest 2002; 121: 708-714.

65 Yan S, Kaminski D, Sliwinski P. Reliability of inspiratory capacity for estimating end-expiratory lung volume changes during exercise in patients with chronic obstructive pulmonary disease. Am J Respir Crit Care Med 1997; 156: $55-59$.

66 American Thoracic Society. Single-breath carbon monoxide diffusing capacity (transfer factor). Recommendations for a standard technique -- 1995 update. Am J Respir Crit Care Med 1995; 152: 2185-2198.

67 Cramer D. Standardization of the measurement of transfer factor. Eur Respir J 1993; 6: 1577-1578.

68 Macintyre N, Crapo RO, Viegi G, et al. Standardisation of the single-breath determination of carbon monoxide uptake in the lung. Eur Respir J 2005; 26: 720-735.

69 Paoletti P, Viegi G, Pistelli G, et al. Reference equations for the single-breath diffusing capacity. A cross-sectional analysis and effect of body size and age. Am Rev Respir Dis 1985; 132: 806-813.

70 Knudson RJ, Kaltenborn WT, Knudson DE, Burrows B. The single-breath carbon monoxide diffusing capacity. Reference equations derived from a healthy nonsmoking population and effects of hematocrit. Am Rev Respir Dis 1987; 135: 805-811.

71 Roca J, Rodriguez-Roisin R, Cobo E, Burgos F, Perez J, Clausen JL. Single-breath carbon monoxide diffusing capacity prediction equations from a Mediterranean population. Am Rev Respir Dis 1990; 141: 1026-1032.

72 Cerveri I, Dore R, Corsico A, et al. Assessment of emphysema in COPD: a functional and radiologic study. Chest 2004; 125: 1714-1718.

73 Goldberg R, Hillberg R, Reinecker L, Goldstein R. Evaluation of patients with severe pulmonary disease before and after pulmonary rehabilitation. Disabil Rehabil 2004; 26: 641-648.

74 Trow TK. Lung-volume reduction surgery for severe emphysema: appraisal of its current status. Curr Opin Pulm Med 2004; 10: 128-132.

75 Meyers BF, Yusen RD, Guthrie TJ, et al. Results of lung volume reduction surgery in patients meeting a national emphysema treatment trial high-risk criterion. J Thorac Cardiovasc Surg 2004; 127: 829-835.

76 Wanger J, Irvin C. Comparability of pulmonary function results from 13 laboratories in a metropolitan area. Respir Care 1991; 36: 1375-1382.

77 Gaensler EA, Smith AA. Attachment for automated single breath diffusing capacity measurement. Chest 1973; 63: 136-145.

78 Punjabi NM, Shade D, Patel AM, Wise RA. Measurement variability in single-breath diffusing capacity of the lung. Chest 2003; 123: 1082-1089.

79 Hathaway EH, Tashkin DP, Simmons MS. Intraindividual variability in serial measurements of $D \mathrm{~L}, \mathrm{CO}$ and alveolar volume over one year in eight healthy subjects using three independent measuring systems. Am Rev Respir Dis 1989; 140: 1818-1822.

80 Bolliger CT, Koegelenberg CF, Kendal R. Preoperative assessment for lung cancer surgery. Curr Opin Pulm Med 2005; 11: 301-306.
81 Schluchter MD, Stoller JK, Barker AF, et al. Feasibility of a clinical trial of augmentation therapy for $\alpha_{1}$-antitrypsin deficiency. The $\alpha_{1}$-Antitrypsin Deficiency Registry Study Group. Am J Respir Crit Care Med 2000; 161: 796-801.

82 Stoller JK, Snider GL, Brantly ML, et al. [American Thoracic Society/European Respiratory Society Statement: Standards for the diagnosis and management of individuals with $\alpha_{1}$-antitrypsin deficiency.] Pneumologie 2005; 59: 36-68.

83 Eaton T, Rudkin S, Garrett JE. The clinical utility of arterialized earlobe capillary blood in the assessment of patients for long-term oxygen therapy. Respir Med 2001; 95: 655-660.

84 Fajac I, Texereau J, Rivoal V, Dessanges JF, DinhXuan AT, l'Ava-Santucci J. Blood gas measurement during exercise: a comparative study between arterialized earlobe sampling and direct arterial puncture in adults. Eur Respir J 1998; 11: 712-715.

85 Williams AJ. ABC of oxygen: assessing and interpreting arterial blood gases and acid-base balance. BMJ 1998; 317: 1213-1216.

86 Raff H. The significance of the blood gas analyzer. J Appl Physiol 2004; 97: 1597-1598.

87 Severinghaus JW. First electrodes for blood $\mathrm{PO}_{2}$ and $\mathrm{PCO}_{2}$ determination. J Appl Physiol 2004; 97: 1599-1600.

88 Aoyagi T. Pulse oximetry: its invention, theory, and future. J Anesth 2003; 17: 259-266.

89 Jubran A. Pulse oximetry. Intensive Care Med 2004; 30: 2017-2020.

90 Sinex JE. Pulse oximetry: principles and limitations. Am J Emerg Med 1999; 17: 59-67.

91 Welch J. Pulse oximeters. Biomed Instrum Technol 2005; 39: 125-130.

92 Cerveri I, Zoia MC, Fanfulla F, et al. Reference values of arterial oxygen tension in the middle-aged and elderly. Am J Respir Crit Care Med 1995; 152: 934-941.

93 Hardie JA, Vollmer WM, Buist AS, Ellingsen I, Morkve O. Reference values for arterial blood gases in the elderly. Chest 2004; 125: 2053-2060.

94 Soler-Cataluña JJ, Martínez-García MA, Román Sánchez P, Salcedo E, Navarro M, Ochando R. Severe acute exacerbations and mortality in patients with chronic obstructive pulmonary disease. Thorax 2005; 60: 925-931.

95 Gunen H, Hacievliyagil SS, Kosar F, et al. Factors affecting survival of hospitalised patients with COPD. Eur Respir J 2005; 26: 234-241.

96 Gay PC. Chronic obstructive pulmonary disease and sleep. Respir Care 2004; 49: 39-51.

97 Littner MR. Portable monitoring in the diagnosis of the obstructive sleep apnea syndrome. Semin Respir Crit Care Med 2005; 26: 56-67.

98 Datta D, Vitale A, Lahiri B, ZuWallack R. An evaluation of nebulized levalbuterol in stable COPD. Chest 2003; 124 844-849.

99 Sans-Torres J, Domingo C, Moron A, Rue M, Marin A. Long-term effects of almitrine bismesylate in COPD patients with chronic hypoxaemia. Respir Med 2003; 97: 599-605.

100 Cazzola M, Santus P, Matera MG, et al. A single high dose of formoterol is as effective as the same dose administered 
in a cumulative manner in patients with acute exacerbation of COPD. Respir Med 2003; 97: 458-462.

101 Cazzola M, Santus P, di Marco F, et al. Bronchodilator effect of an inhaled combination therapy with salmeterol + fluticasone and formoterol + budesonide in patients with COPD. Respir Med 2003; 97: 453-457.

102 Jaen Díaz JI, de Castro MC, Cordero GB, GarcíaSalamanca MJ, Callejas PS, López DC. [Effectiveness of treatment with tiotropium in patients who suffer from COPD]. Med Clin (Barc) 2005; 124: 1-3.

103 Soto FJ, Varkey B. Evidence-based approach to acute exacerbations of COPD. Curr Opin Pulm Med 2003; 9: 117-124.

104 Higgins D. Pulse oximetry. Nurs Times 2005; 101: 34-35.

105 Lawrence JP. Advances and new insights in monitoring. Thorac Surg Clin 2005; 15: 55-70.

106 Hildick-Smith DJ, Lowe MD, Walsh JT, et al. Coronary angiography from the radial artery - experience, complications and limitations. Int J Cardiol 1998; 64: 231-239.

107 Fabbri LM, Hurd SS. Global Strategy for the Diagnosis, Management and Prevention of COPD: 2003 update. Eur Respir J 2003; 22: 1-2.

108 Arnold R, Ranchor AV, Koeter GH, de Jongste MJ, Sanderman R. Consequences of chronic obstructive pulmonary disease and chronic heart failure: the relationship between objective and subjective health. Soc Sci Med 2005; 61: 2144-2154.

109 Jones P, Lareau S, Mahler DA. Measuring the effects of COPD on the patient. Respir Med 2005; 99: Suppl. 2, S11-S18.

110 Hickey AM, Bury G, O’Boyle CA, Bradley F, O'Kelly FD, Shannon W. A new short form individual quality of life measure (SEIQoL-DW): application in a cohort of individuals with HIV/AIDS. BMJ 1996; 313: 29-33.

111 American Thoracic Society. Quality of Life Resource. www.atsqol.org Date last accessed: October 25, 2007.

112 Ware JE Jr, Gandek B. Overview of the SF-36 Health Survey and the International Quality of Life Assessment (IQoLA) Project. J Clin Epidemiol 1998; 51: 903-912.

113 Bergner M. The sickness impact profile. In: Walker SR, ed. Quality of Life: Assessment and Application. Boston, M.T.P., 1987; pp. 79-94.

114 Hunt SM, McEwen J. The development of a subjective health indicator. Sociol Health Illn 1980; 2: 231-246.

115 Guyatt GH, Berman LB, Townsend M, Pugsley SO, Chambers LW. A measure of quality of life for clinical trials in chronic lung disease. Thorax 1987; 42: 773-778.

116 Schunemann HJ, Goldstein R, Mador MJ, et al. A randomised trial to evaluate the self-administered standardised chronic respiratory questionnaire. Eur Respir J 2005; 25: 31-40.

117 Puhan MA, Behnke M, Frey M, et al. Self-administration and interviewer-administration of the German Chronic Respiratory Questionnaire: instrument development and assessment of validity and reliability in two randomised studies. Health Qual Life Outcomes 2004; 2: 1.

118 Wyrwich KW, Tierney WM, Wolinsky FD. Further evidence supporting an SEM-based criterion for identifying meaningful intra-individual changes in health-related quality of life. J Clin Epidemiol 1999; 52: 861-873.

119 Jones PW, Quirk FH, Baveystock CM, Littlejohns P. A self-complete measure of health status for chronic airflow limitation. The St. George's Respiratory Questionnaire. Am Rev Respir Dis 1992; 145: 1321-1327.

120 Wilson CB, Jones PW, O'Leary CJ, Cole PJ, Wilson R. Validation of the St. George's Respiratory Questionnaire in bronchiectasis. Am J Respir Crit Care Med 1997; 156: 536-541.

121 Maille AR, Koning CJ, Zwinderman AH, Willems LN, Dijkman JH, Kaptein AA. The development of the "Quality-of-life for Respiratory Illness Questionnaire (QoL-RIQ)": a disease-specific quality-of-life questionnaire for patients with mild to moderate chronic nonspecific lung disease. Respir Med 1997; 91: 297-309.

122 Jacobs JE, Maille AR, Akkermans RP, van Weel C, Grol RP. Assessing the quality of life of adults with chronic respiratory diseases in routine primary care: construction and first validation of the 10-Item Respiratory Illness Questionnaire-monitoring 10 (RIQMON10). Qual Life Res 2004; 13: 1117-1127.

123 Barley EA, Quirk FH, Jones PW. Asthma health status measurement in clinical practice: validity of a new short and simple instrument. Respir Med 1998; 92: 1207-1214.

124 Hajiro T, Nishimura K, Jones PW, et al. A novel, short, and simple questionnaire to measure health-related quality of life in patients with chronic obstructive pulmonary disease. Am J Respir Crit Care Med 1999; 159: 1874-1878.

125 Alemayehu B, Aubert RE, Feifer RA, Paul LD. Comparative analysis of two quality-of-life instruments for patients with chronic obstructive pulmonary disease. Value Health 2002; 5: 436-441.

126 Hyland ME, Bott J, Singh S, Kenyon CA. Domains, constructs and the development of the breathing problems questionnaire. Qual Life Res 1994; 3: 245-256.

127 Hyland ME, Singh SJ, Sodergren SC, Morgan MP. Development of a shortened version of the Breathing Problems Questionnaire suitable for use in a pulmonary rehabilitation clinic: a purpose-specific, disease-specific questionnaire. Qual Life Res 1998; 7: 227-233.

128 Carone M, Bertolotti G, Anchisi F, Zotti AM, Donner CF, Jones PW. Analysis of factors that characterize health impairment in patients with chronic respiratory failure. Quality of Life in Chronic Respiratory Failure Group. Eur Respir J 1999; 13: 1293-1300.

129 van der Molen T, Willemse BW, Schokker $S$, ten Hacken NH, Postma DS, Juniper EF. Development, validity and responsiveness of the Clinical COPD Questionnaire. Health Qual Life Outcomes 2003; 1: 13.

130 Lareau SC, Meek PM, Roos PJ. Development and testing of the modified version of the pulmonary functional status and dyspnoea questionnaire (PFSDQ-M). Heart Lung 1998; 27: 159-168.

131 Weaver TE, Narsavage GL, Guilfoyle MJ. The development and psychometric evaluation of the Pulmonary Functional Status Scale: an instrument to assess functional status in pulmonary disease. J Cardiopulm Rehabil 1998; 18: 105-111.

132 Nouri FM, Lincoln NB. An extended activities of daily living scale for stroke patients. Clin Rehabil 1987; 1: 301-305.

133 Okubadejo AA, O'Shea L, Jones PW, Wedzicha JA. Home assessment of activities of daily living in patients with 
severe chronic obstructive pulmonary disease on longterm oxygen therapy. Eur Respir J 1997; 10: 1572-1575.

134 Garrod R, Bestall JC, Paul EA, Wedzicha JA, Jones PW. Development and validation of a standardized measure of activity of daily living in patients with severe COPD: the London Chest Activity of Daily Living scale (LCADL). Respir Med 2000; 94: 589-596.

135 Schunemann HJ, Goldstein R, Mador MJ, et al. Do clinical marker states improve responsiveness and construct validity of the standard gamble and feeling thermometer: a randomized multi-center trial in patients with chronic respiratory disease. Qual Life Res 2006; 15: 1-14.

136 Schunemann HJ, Griffith L, Stubbing D, Goldstein R, Guyatt GH. A clinical trial to evaluate the measurement properties of 2 direct preference instruments administered with and without hypothetical marker states. Med Decis Making 2003; 23: 140-149.

137 Schunemann HJ, Griffith L, Jaeschke R, Goldstein R, Stubbing D, Guyatt GH. Evaluation of the minimal important difference for the feeling thermometer and the St. George's Respiratory Questionnaire in patients with chronic airflow obstruction. J Clin Epidemiol 2003; 56: 1170-1176.

138 Puhan MA, Guyatt GH, Montori VM, et al. The standard gamble demonstrated lower reliability than the feeling thermometer. J Clin Epidemiol 2005; 58: 458-465.

139 Kaplan RM, Atkins CJ, Timms R. Validity of a quality of well-being scale as an outcome measure in chronic obstructive pulmonary disease. J Chronic Dis 1984; 37: 85-95.

140 EuroQol Group. EQ-5D. http://euroqol.org Date last accessed: October 25, 2007.

141 Mahler DA. Measurement of dyspnea: clinical ratings. In: Mahler DA, ed. Dyspnea: Mechanisms, Measurement and Management. 2nd Edn. New York, Taylor \& Francis Inc., 2005; pp. 147-164.

142 Fletcher CM, Elmes PC, Fairbairn AS, Wood CH. The significance of respiratory symptoms and the diagnosis of chronic bronchitis in a working population. BMJ 1959; 5147: 257-266.

143 Mahler DA, Weinberg DH, Wells CK, Feinstein AR. The measurement of dyspnea. Contents, interobserver agreement, and physiologic correlates of two new clinical indexes. Chest 1984; 85: 751-758.

144 Bestall JC, Paul EA, Garrod R, Garnham R, Jones PW, Wedzicha JA. Usefulness of the Medical Research Council (MRC) dyspnoea scale as a measure of disability in patients with chronic obstructive pulmonary disease. Thorax 1999; 54: 581-586.

145 Hajiro T, Nishimura K, Tsukino M, Ikeda A, Koyama H, Izumi T. Analysis of clinical methods used to evaluate dyspnea in patients with chronic obstructive pulmonary disease. Am J Respir Crit Care Med 1998; 158: 1185-1189.

146 Ries AL, Kaplan RM, Blumberg E. Use of factor analysis to consolidate multiple outcome measures in chronic obstructive pulmonary disease. J Clin Epidemiol 1991; 44: 497-503.

147 Mahler DA, Harver A. A factor analysis of dyspnea ratings, respiratory muscle strength, and lung function in patients with chronic obstructive pulmonary disease. Am Rev Respir Dis 1992; 145: 467-470.
148 Mahler DA, Ward J, Fierro-Carrion G, et al. Development of self-administered versions of modified baseline and transition dyspnea indexes in COPD. J COPD 2004; 1: 1-8.

149 Witek TJ Jr, Mahler DA. Meaningful effect size and patterns of response of the transition dyspnea index. J Clin Epidemiol 2003; 56: 248-255.

150 Witek TJ Jr, Mahler DA. Minimal important difference of the transition dyspnoea index in a multinational clinical trial. Eur Respir J 2003; 21: 267-272.

151 Mahler DA, Tomlinson D, Olmstead EM, Tosteson AN, O'Connor GT. Changes in dyspnea, health status, and lung function in chronic airway disease. Am J Respir Crit Care Med 1995; 151: 61-65.

152 Casaburi R, Mahler DA, Jones PW, et al. A long-term evaluation of once-daily inhaled tiotropium in chronic obstructive pulmonary disease. Eur Respir J 2002; 19: 217-224.

153 Mahler DA. Dyspnea. In: Celli BR, ed. Pharmacotherapy of COPD. New York, Marcel Dekker Inc., 2003; pp. 145-158.

154 Brusasco V, Hodder R, Miravitlles M, Korducki L, Towse L, Kesten S. Health outcomes following treatment for six months with once daily tiotropium compared with twice daily salmeterol in patients with COPD. Thorax 2003; 58: 399-404.

155 ZuWallack RL, Mahler DA, Reilly D, et al. Salmeterol plus theophylline combination therapy in the treatment of COPD. Chest 2001; 119: 1661-1670.

156 Vincken W, van Noord JA, Greefhorst AP, et al. Improved health outcomes in patients with COPD during $1 \mathrm{yr}^{\prime} \mathrm{s}$ treatment with tiotropium. Eur Respir J 2002; 19: 209-216.

157 Donohue JF, Kalberg C, Emmett A, Merchant K, Knobil K. A short-term comparison of fluticasone propionate/ salmeterol with ipratropium bromide/albuterol for the treatment of COPD. Treat Respir Med 2004; 3: 173-181.

158 Donohue JF, van Noord JA, Bateman ED, et al. A 6month, placebo-controlled study comparing lung function and health status changes in COPD patients treated with tiotropium or salmeterol. Chest 2002; 122: 47-55.

159 Hanania NA, Darken P, Horstman D, et al. The efficacy and safety of fluticasone propionate $(250 \mu \mathrm{g}) /$ salmeterol $(50 \mu \mathrm{g})$ combined in the Diskus inhaler for the treatment of COPD. Chest 2003; 124: 834-843.

160 Mahler DA, Wire P, Horstman D, et al. Effectiveness of fluticasone propionate and salmeterol combination delivered via the Diskus device in the treatment of chronic obstructive pulmonary disease. Am J Respir Crit Care Med 2002; 166: 1084-1091.

161 Aaron SD, Vandemheen KL, Hebert P, et al. Outpatient oral prednisone after emergency treatment of chronic obstructive pulmonary disease. N Engl J Med 2003; 348: 2618-2625.

162 Guyatt GH, Townsend M, Pugsley SO, et al. Bronchodilators in chronic air-flow limitation. Effects on airway function, exercise capacity, and quality of life. Am Rev Respir Dis 1987; 135: 1069-1074.

163 Mahler DA, Donohue JF, Barbee RA, et al. Efficacy of salmeterol xinafoate in the treatment of COPD. Chest 1999; 115: 957-965.

164 Rennard SI, Anderson W, ZuWallack R, et al. Use of a long-acting inhaled $\beta_{2}$-adrenergic agonist, salmeterol xinafoate, in patients with chronic obstructive pulmonary disease. Am J Respir Crit Care Med 2001; 163: 1087-1092. 
165 Rutten-van Mölken M, Roos B, van Noord JA. An empirical comparison of the St George's Respiratory Questionnaire (SGRQ) and the Chronic Respiratory Disease Questionnaire (CRQ) in a clinical trial setting. Thorax 1999; 54: 995-1003.

166 Borg GA. Psychophysical bases of perceived exertion. Med Sci Sports Exerc 1982; 14: 377-381.

167 Mahler DA, Mejia-Alfaro R, Ward J, Baird JC. Continuous measurement of breathlessness during exercise: validity, reliability, and responsiveness. J Appl Physiol 2001; 90: 2188-2196.

168 Fierro-Carrion G, Mahler DA, Ward J, Baird JC. Comparison of continuous and discrete measurements of dyspnea during exercise in patients with COPD and normal subjects. Chest 2004; 125: 77-84.

169 Mahler DA, Fierro-Carrion G, Mejia-Alfaro R, Ward J, Baird JC. Responsiveness of continuous ratings of dyspnea during exercise in patients with COPD. Med Sci Sports Exerc 2005; 37: 529-535.

170 Mahler DA. Clinical measurement of dyspnea. In: Mahler DA, ed. Dyspnea. Lung Biology in Health and Disease. New York, Marcel Dekker Inc., 1998; pp. 149-198.

171 Hamilton AL, Killian KJ, Summers E, Jones NL. Symptom intensity and subjective limitation to exercise in patients with cardiorespiratory disorders. Chest 1996; 110: 1255-1263.

172 Teramoto S, Fukuchi Y, Orimo H. Effects of inhaled anticholinergic drug on dyspnea and gas exchange during exercise in patients with chronic obstructive pulmonary disease. Chest 1993; 103: 1774-1782.

173 Ayers ML, Mejia R, Ward J, Lentine T, Mahler DA. Effectiveness of salmeterol versus ipratropium bromide on exertional dyspnoea in COPD. Eur Respir J 2001; 17: 1132-1137.

174 Jones PW. Health status measurement in chronic obstructive pulmonary disease. Thorax 2001; 56: 880-887.

175 Jones PW, Quirk FH, Baveystock CM. Why quality of life measures should be used in the treatment of patients with respiratory illness. Monaldi Arch Chest Dis 1994; 49: 79-82.

176 Campbell M, Eliraz A, Johansson G, et al. Formoterol for maintenance and as-needed treatment of chronic obstructive pulmonary disease. Respir Med 2005; 99: 1511-1520.

177 Franciosi LG, Page CP, Celli BR, et al. Markers of disease severity in chronic obstructive pulmonary disease. Pulm Pharmacol Ther 2006; 19: 189-199.

178 Seemungal TA, Donaldson GC, Paul EA, Bestall JC, Jeffries DJ, Wedzicha JA. Effect of exacerbation on quality of life in patients with chronic obstructive pulmonary disease. Am J Respir Crit Care Med 1998; 157: 1418-1422.

179 Miravitlles M, Ferrer M, Pont A, et al. Effect of exacerbations on quality of life in patients with chronic obstructive pulmonary disease: a 2 year follow up study. Thorax 2004; 59: 387-395.

180 Doll H, Miravitlles M. Health-related QoL in acute exacerbations of chronic bronchitis and chronic obstructive pulmonary disease: a review of the literature. Pharmacoeconomics 2005; 23: 345-363.

181 Schmier JK, Halpern MT, Higashi MK, Bakst A. The quality of life impact of acute exacerbations of chronic bronchitis (AECB): a literature review. Qual Life Res 2005; 14: 329-347.

182 Burrows B, Earle RH. Course and prognosis of chronic obstructive lung disease. A prospective study of 200 patients. N Engl J Med 1969; 280: 397-404.

183 Hurst JR, Donaldson GC, Perera WR, et al. Use of plasma biomarkers at exacerbation of chronic obstructive pulmonary disease. Am J Respir Crit Care Med 2006; 174: 867-874.

184 Pauwels R, Calverley P, Buist AS, et al. COPD exacerbations: the importance of a standard definition. Respir Med 2004; 98: 99-107.

185 Anthonisen NR, Manfreda J, Warren CP, Hershfield ES, Harding GK, Nelson NA. Antibiotic therapy in exacerbations of chronic obstructive pulmonary disease. Ann Intern Med 1987; 106: 196-204.

186 Rodriguez-Roisin R. Toward a consensus definition for COPD exacerbations. Chest 2000; 117: Suppl. 2, 398S-401S

187 Decramer M, Rutten-van Mölken M, Dekhuijzen PN, et al. Effects of $N$-acetylcysteine on outcomes in chronic obstructive pulmonary disease (Bronchitis Randomized on NAC Cost-Utility Study, BRONCUS): a randomised placebo-controlled trial. Lancet 2005; 365: 1552-1560.

188 Fabbri L, Beghe B, Caramori G, Papi A, Saetta M. Similarities and discrepancies between exacerbations of asthma and chronic obstructive pulmonary disease. Thorax 1998; 53: 803-808.

189 Wilkinson TM, Donaldson GC, Hurst JR, Seemungal TA, Wedzicha JA. Early therapy improves outcomes of exacerbations of chronic obstructive pulmonary disease. Am J Respir Crit Care Med 2004; 169: 1298-1303.

190 Burge PS, Calverley PM, Jones PW, Spencer S, Anderson JA, Maslen TK. Randomised, double blind, placebo controlled study of fluticasone propionate in patients with moderate to severe chronic obstructive pulmonary disease: the ISOLDE trial. BMJ 2000; 320: 1297-1303.

191 Calverley PM, Boonsawat W, Cseke Z, Zhong N, Peterson S, Olsson H. Maintenance therapy with budesonide and formoterol in chronic obstructive pulmonary disease. Eur Respir J 2003; 22: 912-919.

192 Kessler R, Stahl E, Vogelmeier C, et al. Patient understanding, detection, and experience of COPD exacerbations: an observational, interview-based study. Chest 2006; 130: 133-142.

193 Allegra L, Grassi C, Grossi E, et al. Ruolo degli antibiotici nel trattamento delle riacutizzazioni della bronchite cronica: risultati di uno studio italiano multicentrico. [Role of antibiotics in the treatment of acute exacerbations of chronic bronchitis: results from an Italian multicentre trial.] Ital J Chest Dis 1991; 45: 138-148.

194 Donaldson GC, Seemungal TA, Patel IS, Lloyd-Owen SJ, Wilkinson TM, Wedzicha JA. Longitudinal changes in the nature, severity and frequency of COPD exacerbations. Eur Respir J 2003; 22: 931-936.

195 Szafranski W, Cukier A, Ramirez A, et al. Efficacy and safety of budesonide/formoterol in the management of chronic obstructive pulmonary disease. Eur Respir J 2003; 21: 74-81.

196 Vestbo J. The TORCH (towards a revolution in COPD health) survival study protocol. Eur Respir J 2004; 24: 206-210. 
197 Calverley P, Pauwels R, Vestbo J, et al. Combined salmeterol and fluticasone in the treatment of chronic obstructive pulmonary disease: a randomised controlled trial. Lancet 2003; 361: 449-456.

198 Jones PW, Willits LR, Burge PS, Calverley PM. Disease severity and the effect of fluticasone propionate on chronic obstructive pulmonary disease exacerbations. Eur Respir J 2003; 21: 68-73.

199 Gompertz S, Bayley DL, Hill SL, Stockley RA. Relationship between airway inflammation and the frequency of exacerbations in patients with smoking related COPD. Thorax 2001; 56: 36-41.

200 Spencer S, Calverley PM, Burge PS, Jones PW. Impact of preventing exacerbations on deterioration of health status in COPD. Eur Respir J 2004; 23: 698-702.

201 Miravitlles M, Murio C, Guerrero T. Factors associated with relapse after ambulatory treatment of acute exacerbations of chronic bronchitis. DAFNE Study Group. Eur Respir J 2001; 17: 928-933.

202 Suissa S. Statistical treatment of exacerbations in therapeutic trials of chronic obstructive pulmonary disease. Am I Respir Crit Care Med 2006; 173: 842-846.

203 Miravitlles M, Murio C, Guerrero T, Gisbert R. Pharmacoeconomic evaluation of acute exacerbations of chronic bronchitis and COPD. Chest 2002; 121: 1449-1455.

204 Garcia-Aymerich J, Monso E, Marrades RM, et al. Risk factors for hospitalization for a chronic obstructive pulmonary disease exacerbation. EFRAM study. Am J Respir Crit Care Med 2001; 164: 1002-1007.

205 Miravitlles M, Guerrero T, Mayordomo C, SanchezAgudo L, Nicolau F, Segu JL. Factors associated with increased risk of exacerbation and hospital admission in a cohort of ambulatory COPD patients: a multiple logistic regression analysis. The EOLO Study Group. Respiration 2000; 67: 495-501.

206 Antonelli I, Fuso L, De Rosa M, et al. Comorbidity contributes to predict mortality of patients with chronic obstructive pulmonary disease. Eur Respir J 1997; 10: 2794-2800.

207 Dowson LJ, Newall C, Guest PJ, Hill SL, Stockley RA. Exercise capacity predicts health status in $\alpha_{1}$-antitrypsin deficiency. Am J Respir Crit Care Med 2001; 163: 936-941.

208 Clinical exercise testing with reference to lung diseases: indications, standardization and interpretation strategies. ERS Task Force on Standardization of Clinical Exercise Testing. European Respiratory Society. Eur Respir J 1997; 10: 2662-2689.

209 ATS/ACCP Statement on cardiopulmonary exercise testing. Am J Respir Crit Care Med 2003; 167: 211-277.

210 Saey D, Michaud A, Couillard A, et al. Contractile fatigue, muscle morphometry, and blood lactate in chronic obstructive pulmonary disease. Am J Respir Crit Care Med 2005; 171: 1109-1115.

211 Bowen JB, Votto JJ, Thrall RS, et al. Functional status and survival following pulmonary rehabilitation. Chest 2000; 118: 697-703.

212 Oga T, Nishimura K, Tsukino M, Sato S, Hajiro T. Analysis of the factors related to mortality in chronic obstructive pulmonary disease: role of exercise capacity and health status. Am J Respir Crit Care Med 2003; 167: 544-549.
213 Oga T, Nishimura K, Tsukino M, Hajiro T, Ikeda A, Izumi T. The effects of oxitropium bromide on exercise performance in patients with stable chronic obstructive pulmonary disease. A comparison of three different exercise tests. Am J Respir Crit Care Med 2000; 161: 1897-1901.

214 Palange P, Crimi E, Pellegrino R, Brusasco V. Supplemental oxygen and heliox: "new" tools for exercise training in chronic obstructive pulmonary disease. Curr Opin Pulm Med 2005; 11: 145-148.

215 Butland RJ, Pang J, Gross ER, Woodcock AA, Geddes DM. Two-, six-, and 12-minute walking tests in respiratory disease. Br Med J (Clin Res Ed) 1982; 284: 1607-1608.

216 Palange $\mathrm{P}$, Forte S, Onorati P, Manfredi F, Serra P, Carlone S. Ventilatory and metabolic adaptations to walking and cycling in patients with COPD. J Appl Physiol 2000; 88: 1715-1720.

217 Man WD, Soliman MG, Gearing J, et al. Symptoms and quadriceps fatigability after walking and cycling in chronic obstructive pulmonary disease. Am J Respir Crit Care Med 2003; 168: 562-567.

218 Fishman A, Martinez F, Naunheim K, et al. A randomized trial comparing lung-volume-reduction surgery with medical therapy for severe emphysema. $N$ Engl J Med 2003; 348: 2059-2073.

219 Sue DY, Wasserman K, Moricca RB, Casaburi R. Metabolic acidosis during exercise in patients with chronic obstructive pulmonary disease. Use of the Vslope method for anaerobic threshold determination. Chest 1988; 94: 931-938.

220 Aliverti A, Stevenson N, Dellaca RL, Lo MA, Pedotti A, Calverley PM. Regional chest wall volumes during exercise in chronic obstructive pulmonary disease. Thorax 2004; 59: 210-216.

221 Myers J, Prakash M, Froelicher V, Do D, Partington S, Atwood JE. Exercise capacity and mortality among men referred for exercise testing. N Engl J Med 2002; 346: 793-801.

222 Pellegrino R, Villosio C, Milanese U, Garelli G, Rodarte JR, Brusasco V. Breathing during exercise in subjects with mild-to-moderate airflow obstruction: effects of physical training. J Appl Physiol 1999; 87: 1697-1704.

223 Casaburi R, Porszasz J, Burns MR, Carithers ER, Chang RS, Cooper CB. Physiologic benefits of exercise training in rehabilitation of patients with severe chronic obstructive pulmonary disease. Am J Respir Crit Care Med 1997; 155: 1541-1551.

224 Martinez FJ, de Oca MM, Whyte RI, Stetz J, Gay SE, Celli BR. Lung-volume reduction improves dyspnea, dynamic hyperinflation, and respiratory muscle function. Am J Respir Crit Care Med 1997; 155: 1984-1990.

225 Somfay A, Porszasz J, Lee SM, Casaburi R. Doseresponse effect of oxygen on hyperinflation and exercise endurance in nonhypoxaemic COPD patients. Eur Respir J 2001; 18: 77-84.

226 O'Donnell DE, D'Arsigny C, Webb KA. Effects of hyperoxia on ventilatory limitation during exercise in advanced chronic obstructive pulmonary disease. Am J Respir Crit Care Med 2001; 163: 892-898.

227 Palange P, Valli G, Onorati P, et al. Effect of heliox on lung dynamic hyperinflation, dyspnea, and exercise 
endurance capacity in COPD patients. J Appl Physiol 2004; 97: 1637-1642.

228 McGavin CR, Gupta SP, McHardy GJ. Twelve-minute walking test for assessing disability in chronic bronchitis. BMJ 1976; 1: 822-823.

229 Morgan AD, Peck DF, Buchanan DR, McHardy GJ. Effect of attitudes and beliefs on exercise tolerance in chronic bronchitis. Br Med J (Clin Res Ed) 1983; 286: 171-173.

230 Sciurba F, Criner GJ, Lee SM, et al. Six-minute walk distance in chronic obstructive pulmonary disease: reproducibility and effect of walking course layout and length. Am J Respir Crit Care Med 2003; 167: 1522-1527.

231 Guyatt GH, Sullivan MJ, Thompson PJ, et al. The 6minute walk: a new measure of exercise capacity in patients with chronic heart failure. Can Med Assoc J 1985; 132: 919-923.

232 Knox AJ, Morrison JF, Muers MF. Reproducibility of walking test results in chronic obstructive airways disease. Thorax 1988; 43: 388-392.

233 Gerardi DA, Lovett L, Benoit-Connors ML, Reardon JZ, ZuWallack RL. Variables related to increased mortality following outpatient pulmonary rehabilitation. Eur Respir J 1996; 9: 431-435.

234 Geddes D, Davies M, Koyama H, et al. Effect of lungvolume-reduction surgery in patients with severe emphysema. N Engl J Med 2000; 343: 239-245.

235 Young J, Fry-Smith A, Hyde C. Lung volume reduction surgery (LVRS) for chronic obstructive pulmonary disease (COPD) with underlying severe emphysema. Thorax 1999; 54: 779-789.

236 Griffiths TL, Burr ML, Campbell IA, et al. Results at 1 year of outpatient multidisciplinary pulmonary rehabilitation: a randomised controlled trial. Lancet 2000; 355: 362-368.

237 Leach RM, Davidson AC, Chinn S, Twort CH, Cameron IR, Bateman NT. Portable liquid oxygen and exercise ability in severe respiratory disability. Thorax 1992; 47: 781-789.

238 Corris PA, Neville E, Nariman S, Gibson GJ. Doseresponse study of inhaled salbutamol powder in chronic airflow obstruction. Thorax 1983; 38: 292-296.

239 Onorati P, Antonucci R, Valli G, et al. Non-invasive evaluation of gas exchange during a shuttle walking test versus a 6-min walking test to assess exercise tolerance in COPD patients. Eur J Appl Physiol 2003; 89: 331-336.

240 Singh SJ, Morgan MD, Scott S, Walters D, Hardman AE. Development of a shuttle walking test of disability in patients with chronic airways obstruction. Thorax 1992; 47: 1019-1024.

241 Revill SM, Morgan MD, Singh SJ, Williams J, Hardman AE. The endurance shuttle walk: a new field test for the assessment of endurance capacity in chronic obstructive pulmonary disease. Thorax 1999; 54: 213-222.

242 Man WD, Polkey MI, Donaldson N, Gray BJ, Moxham J. Community pulmonary rehabilitation after hospitalisation for acute exacerbations of chronic obstructive pulmonary disease: a randomised controlled study. BMJ 2004; 329: 1209.

243 Fuld JP, Kilduff LP, Neder JA, et al. Creatine supplementation during pulmonary rehabilitation in chronic obstructive pulmonary disease. Thorax 2005; 60: 531-537.
244 Steiner MC, Barton RL, Singh SJ, Morgan MD. Nutritional enhancement of exercise performance in chronic obstructive pulmonary disease: a randomised controlled trial. Thorax 2003; 58: 745-751.

245 Eiser N, Willsher D, Dore CJ. Reliability, repeatability and sensitivity to change of externally and self-paced walking tests in COPD patients. Respir Med 2003; 97: 407-414.

246 Continuous or nocturnal oxygen therapy in hypoxemic chronic obstructive lung disease: a clinical trial. Nocturnal Oxygen Therapy Trial Group. Ann Intern Med 1980; 93: 391-398.

247 Long-term domiciliary oxygen therapy in chronic hypoxic cor pulmonale complicating chronic bronchitis and emphysema. Report of the Medical Research Council Working Party. Lancet 1981; 1: 681-686.

248 Sin DD, Wu L, Anderson JA, et al. Inhaled corticosteroids and mortality in chronic obstructive pulmonary disease. Thorax 2005; 60: 992-997.

249 Hansell AL, Walk JA, Soriano JB. What do chronic obstructive pulmonary disease patients die from? A multiple cause coding analysis. Eur Respir J 2003; 22: 809-814.

250 Camilli AE, Robbins DR, Lebowitz MD. Death certificate reporting of confirmed airways obstructive disease. Am J Epidemiol 1991; 133: 795-800.

251 Anthonisen NR, Skeans MA, Wise RA, Manfreda J, Kanner RE, Connett JE. The effects of a smoking cessation intervention on 14.5-year mortality: a randomized clinical trial. Ann Intern Med 2005; 142: 233-239.

252 Sin DD, Man SF. Chronic obstructive pulmonary disease: a novel risk factor for cardiovascular disease. Can J Physiol Pharmacol 2005; 83: 8-13.

253 Solomon SD, Wang D, Finn P, et al. Effect of candesartan on cause-specific mortality in heart failure patients: the Candesartan in Heart failure Assessment of Reduction in Mortality and morbidity (CHARM) program. Circulation 2004; 110: 2180-2183.

254 Curb JD, McTiernan A, Heckbert SR, et al. Outcomes ascertainment and adjudication methods in the Women's Health Initiative. Ann Epidemiol 2003; 13: Suppl. 9, S122-S128.

255 Remington RD. Who should code cause of death in a clinical trial? Control Clin Trials 1984; 5: 241-244.

256 Walter SD, Cook DJ, Guyatt GH, King D, Troyan S. Outcome assessment for clinical trials: how many adjudicators do we need? Canadian Lung Oncology Group. Control Clin Trials 1997; 18: 27-42.

257 Anthonisen NR, Wright EC, Hodgkin JE. Prognosis in chronic obstructive pulmonary disease. Am Rev Respir Dis 1986; 133: 14-20.

258 Anthonisen NR, Connett JE, Enright PL, Manfreda J. Hospitalizations and mortality in the Lung Health Study. Am J Respir Crit Care Med 2002; 166: 333-339.

259 Celli BR, Cote CG, Marin JM, et al. The body-mass index, airflow obstruction, dyspnea, and exercise capacity index in chronic obstructive pulmonary disease. $N$ Engl J Med 2004; 350: 1005-1012.

260 Hansen EF, Phanareth K, Laursen LC, Kok-Jensen A, Dirksen A. Reversible and irreversible airflow obstruction as predictor of overall mortality in asthma and chronic obstructive pulmonary disease. Am J Respir Crit Care Med 1999; 159: 1267-1271. 
261 Landbo C, Prescott E, Lange P, Vestbo J, Almdal TP. Prognostic value of nutritional status in chronic obstructive pulmonary disease. Am J Respir Crit Care Med 1999; 160: 1856-1861.

262 Mannino DM, Buist AS, Petty TL, Enright PL, Redd SC. Lung function and mortality in the United States: data from the First National Health and Nutrition Examination Survey follow up study. Thorax 2003; 58: 388-393.

263 Nishimura K, Izumi T, Tsukino M, Oga T. Dyspnea is a better predictor of 5-year survival than airway obstruction in patients with COPD. Chest 2002; 121: 1434-1440.

264 Oga T, Nishimura K, Tsukino M, et al. Health status measured with the $\mathrm{CRQ}$ does not predict mortality in COPD. Eur Respir J 2002; 20: 1147-1151.

265 Prescott E, Almdal T, Mikkelsen KL, Tofteng CL, Vestbo J, Lange P. Prognostic value of weight change in chronic obstructive pulmonary disease: results from the Copenhagen City Heart Study. Eur Respir J 2002; 20: 539-544.

266 Schols AM, Slangen J, Volovics L, Wouters EF. Weight loss is a reversible factor in the prognosis of chronic obstructive pulmonary disease. Am J Respir Crit Care Med 1998; 157: 1791-1797.

267 Soriano JB, Vestbo J, Pride NB, Kiri V, Maden C, Maier WC. Survival in COPD patients after regular use of fluticasone propionate and salmeterol in general practice. Eur Respir J 2002; 20: 819-825.

268 Sin DD, Man SF. Why are patients with chronic obstructive pulmonary disease at increased risk of cardiovascular diseases? The potential role of systemic inflammation in chronic obstructive pulmonary disease. Circulation 2003; 107: 1514-1519.

269 Mannino DM, Ford ES, Redd SC. Obstructive and restrictive lung disease and markers of inflammation: data from the Third National Health and Nutrition Examination. Am J Med 2003; 114: 758-762.

270 Cote CG, Celli BR. Pulmonary rehabilitation and the BODE index in COPD. Eur Respir J 2005; 26: 630-636.

271 Imfeld S, Bloch KE, Weder W, Russi EW. The BODE index after lung volume reduction surgery correlates with survival. Chest 2006; 129: 873-878.

272 Gold MR, Siegel JE, Russell LB, Weinstein MC, eds. CostEffectiveness in Health and Medicine. 1st Edn. New York, Oxford University Press, 1996.

273 Drummond MF, O'Brien B, Stoddart GL, Torrance GW, eds. Methods for the Economic Evaluation of Health Care Programmes. 1st Edn. New York, Oxford University Press, 1997.

274 National Institute for Clinical Excellence (NICE). Guide to the Methods of Technology Appraisal. London, NICE, 2004.

275 Molken MP, Van Doorslaer EK, Rutten FF. Economic appraisal of asthma and COPD care: a literature review 1980-1991. Soc Sci Med 1992; 35: 161-175.

276 Ruchlin HS, Dasbach EJ. An economic overview of chronic obstructive pulmonary disease. Pharmacoeconomics 2001; 19: 623-642.

277 Rutten-van Mölken MP, Postma MJ, Joore MA, van Genugten ML, Leidl R, Jager JC. Current and future medical costs of asthma and chronic obstructive pulmonary disease in The Netherlands. Respir Med 1999; 93: 779-787.
278 Sullivan SD, Ramsey SD, Lee TA. The economic burden of COPD. Chest 2000; 117: Suppl. 2, 5S-9S.

279 Strassels SA, Smith DH, Sullivan SD, Mahajan PS. The costs of treating COPD in the United States. Chest 2001; 119: 344-352.

280 Jansson SA, Andersson F, Borg S, Ericsson A, Jonsson E, Lundback B. Costs of COPD in Sweden according to disease severity. Chest 2002; 122: 1994-2002.

281 Miravitlles M, Murio C, Guerrero T, Gisbert R. Costs of chronic bronchitis and COPD: a 1-year follow-up study. Chest 2003; 123: 784-791.

282 Andersson F, Borg S, Jansson SA, et al. The costs of exacerbations in chronic obstructive pulmonary disease (COPD). Respir Med 2002; 96: 700-708.

283 Oostenbrink JB, Rutten-van Mölken MP. Resource use and risk factors in high-cost exacerbations of COPD. Respir Med 2004; 98: 883-891.

284 van den Berg B, Brouwer WB, Koopmanschap MA. Economic valuation of informal care. An overview of methods and applications. Eur J Health Econ 2004; 5: 36-45.

285 Koopmanschap MA. PRODISQ: a modular questionnaire on productivity and disease for economic evaluation studies. Expert Rev Pharmacoeconomics Outcomes Res 2005; 5: 23-28.

286 Koopmanschap MA, Rutten FF, van Ineveld BM, van Roijen L. The friction cost method for measuring indirect costs of disease. J Health Econ 1995; 14: 171-189.

287 Ramsey SD, Patrick DL, Albert RK, Larson EB, Wood DE, Raghu G. The cost-effectiveness of lung transplantation. A pilot study. University of Washington Medical Center Lung Transplant Study Group. Chest 1995; 108: 1594-1601.

288 Al MJ, Koopmanschap MA, van Enckevort PJ, et al. Costeffectiveness of lung transplantation in The Netherlands: a scenario analysis. Chest 1998; 113: 124-130.

289 Groen H, van der BW, Koeter GH, TenVergert EM. Costeffectiveness of lung transplantation in relation to type of end-stage pulmonary disease. Am J Transplant 2004; 4: 1155-1162.

290 Ramsey SD, Berry K, Etzioni R, Kaplan RM, Sullivan SD, Wood DE. Cost effectiveness of lung-volume-reduction surgery for patients with severe emphysema. N Engl J Med 2003; 348: 2092-2102.

291 Anon JM, Garcia de Lorenzo A, Zarazaga A, Gomez-Tello V, Garrido G, Mechanical ventilation of patients on longterm oxygen therapy with acute exacerbations of chronic obstructive pulmonary disease: prognosis and cost-utility analysis. Intensive Care Med 1999; 25: 452-457.

292 Goldstein RS, Gort EH, Guyatt GH, Feeny D. Economic analysis of respiratory rehabilitation. Chest 1997; 112: 370-379.

293 Griffiths TL, Phillips CJ, Davies S, Burr ML, Campbell IA. Cost-effectiveness of an outpatient multidisciplinary pulmonary rehabilitation programme. Thorax 2001; 56: 779-784.

294 Hoogendoorn M, Rutten-van Mölken MP, Hoogenveen RT, et al. A dynamic population model of disease progression in COPD. Eur Respir J 2005; 26: 223-233.

295 van den BG, Rutten-van Mölken MP, Folgering H, van Weel C, van Schayck CP. The economic effects of screening for obstructive airway disease: an economic 
analysis of the DIMCA program. Prev Med 2000; 30: 302-308.

296 Oostenbrink JB, Rutten-van Mölken MP, Al MJ, van Noord JA, Vincken W. One-year cost-effectiveness of tiotropium versus ipratropium to treat chronic obstructive pulmonary disease. Eur Respir J 2004; 23: 241-249.

297 van Hout BA, Al MJ, Gordon GS, Rutten FF. Costs, effects and C/E-ratios alongside a clinical trial. Health Econ 1994; 3: 309-319.

298 Briggs A, Fenn P. Confidence intervals or surfaces? Uncertainty on the cost-effectiveness plane. Health Econ 1998; 7: 723-740.

299 Briggs AH, Wonderling DE, Mooney CZ. Pulling costeffectiveness analysis up by its bootstraps: a nonparametric approach to confidence interval estimation. Health Econ 1997; 6: 327-340.

300 Langley PC. The NICE reference case requirement: implications for drug manufacturers and health systems. Pharmacoeconomics 2004; 22: 267-271.

301 Fenwick E, Claxton K, Sculpher M. Representing uncertainty: the role of cost-effectiveness acceptability curves. Health Econ 2001; 10: 779-787.

302 Sin DD, Golmohammadi K, Jacobs P. Cost-effectiveness of inhaled corticosteroids for chronic obstructive pulmonary disease according to disease severity. Am J Med 2004; 116: 325-331.

303 Borg S, Ericsson A, Wedzicha J, et al. A computer simulation model of the natural history and economic impact of chronic obstructive pulmonary disease. Value Health 2004; 7: 153-167.

304 Oostenbrink JB, Rutten-van Mölken MP, Monz BU, FitzGerald JM. Probabilistic Markov model to assess the cost-effectiveness of bronchodilator therapy in COPD patients in different countries. Value Health 2005; 8: 32-46.

305 Spencer M, Briggs AH, Grossman RF, Rance L. Development of an economic model to assess the cost effectiveness of treatment interventions for chronic obstructive pulmonary disease. Pharmacoeconomics 2005; 23: 619-637.

306 Briggs A, Sculpher M. An introduction to Markov modelling for economic evaluation. Pharmacoeconomics 1998; 13: 397-409.

307 Hogg JC. Pathophysiology of airflow limitation in chronic obstructive pulmonary disease. Lancet 2004; 364: 709-721.

308 Miniati M, Filippi E, Falaschi F, et al. Radiologic evaluation of emphysema in patients with chronic obstructive pulmonary disease. Chest radiography versus high resolution computed tomography. Am J Respir Crit Care Med 1995; 151: 1359-1367.

309 Kinsella M, Muller NL, Staples C, Vedal S, ChanYeung M. Hyperinflation in asthma and emphysema. Assessment by pulmonary function testing and computed tomography. Chest 1988; 94: 286-289.

310 Morrison NJ, Abboud RT, Ramadan F, et al. Comparison of single breath carbon monoxide diffusing capacity and pressure-volume curves in detecting emphysema. Am Rev Respir Dis 1989; 139: 1179-1187.

311 Parr DG, Stoel BC, Stolk J, Stockley RA. Validation of computed tomographic lung densitometry for monitoring emphysema in $\alpha_{1}$-antitrypsin deficiency. Thorax 2006; 61: 485-490.
312 Biernacki W, Ryan M, MacNee W, Flenley DC. Can the quantitative CT scan detect progression of emphysema? Am Rev Respir Dis 1989; 131: A120.

313 Dirksen A, Dijkman JH, Madsen F, et al. A randomized clinical trial of $\alpha_{1}$-antitrypsin augmentation therapy. Am J Respir Crit Care Med 1999; 160: 1468-1472.

314 Dowson LJ, Guest PJ, Stockley RA. Longitudinal changes in physiological, radiological, and health status measurements in $\alpha_{1}$-antitrypsin deficiency and factors associated with decline. Am J Respir Crit Care Med 2001; 164: 1805-1809.

315 Soejima K, Yamaguchi K, Kohda E, et al. Longitudinal follow-up study of smoking-induced lung density changes by high-resolution computed tomography. Am J Respir Crit Care Med 2000; 161: 1264-1273.

316 Nakano Y, Muro S, Sakai H, et al. Computed tomographic measurements of airway dimensions and emphysema in smokers. Correlation with lung function. Am J Respir Crit Care Med 2000; 162: 1102-1108.

317 Orlandi I, Moroni C, Camiciottoli G, et al. Chronic obstructive pulmonary disease: thin-section CT measurement of airway wall thickness and lung attenuation. Radiology 2005; 234: 604-610.

318 Nakano Y, Muller NL, King GG, et al. Quantitative assessment of airway remodeling using high-resolution CT. Chest 2002; 122: Suppl. 6, 271S-275S.

319 O'Donnell RA, Peebles C, Ward JA, et al. Relationship between peripheral airway dysfunction, airway obstruction, and neutrophilic inflammation in COPD. Thorax 2004; 59: 837-842.

320 Hayhurst MD, MacNee W, Flenley DC, et al. Diagnosis of pulmonary emphysema by computerised tomography. Lancet 1984; 2: 320-322.

321 Guenard H, Diallo MH, Laurent F, Vergeret J. Lung density and lung mass in emphysema. Chest 1992; 102: 198-203.

322 Haraguchi M, Shimura S, Hida W, Shirato K. Pulmonary function and regional distribution of emphysema as determined by high-resolution computed tomography. Respiration 1998; 65: 125-129.

323 Heremans A, Verschakelen JA, Van Fraeyenhoven L, Demedts M. Measurement of lung density by means of quantitative CT scanning. A study of correlations with pulmonary function tests. Chest 1992; 102: 805-811.

324 Hartley PG, Galvin JR, Hunninghake GW, et al. Highresolution CT-derived measures of lung density are valid indexes of interstitial lung disease. J Appl Physiol 1994; 76: 271-277.

325 Rienmuller RK, Behr J, Kalender WA, et al. Standardized quantitative high resolution CT in lung diseases. J Comput Assist Tomogr 1991; 15: 742-749.

326 Gould GA, MacNee W, McLean A, et al. CT measurements of lung density in life can quantitate distal airspace enlargement - an essential defining feature of human emphysema. Am Rev Respir Dis 1988; 137: 380-392.

327 Gould GA, Redpath AT, Ryan M, et al. Lung CT density correlates with measurements of airflow limitation and the diffusing capacity. Eur Respir J 1991; 4: 141-146.

328 Dirksen A, Friis M, Olesen KP, Skovgaard LT, Sorensen K. Progress of emphysema in severe $\alpha_{1}$-antitrypsin deficiency as assessed by annual CT. Acta Radiol 1997; 38: 826-832. 
329 Bankier AA, De Maertelaer, V, Keyzer C, Gevenois PA. Pulmonary emphysema: subjective visual grading versus objective quantification with macroscopic morphometry and thin-section CT densitometry. Radiology 1999; 211: 851-858.

330 Gevenois PA, De Maertelaer, V, De Vuyst P, Zanen J, Yernault JC. Comparison of computed density and macroscopic morphometry in pulmonary emphysema. Am J Respir Crit Care Med 1995; 152: 653-657.

331 Gevenois PA, De Vuyst P, Sy M, et al. Pulmonary emphysema: quantitative CT during expiration. Radiology 1996; 199: 825-829.

332 Gevenois PA, De Vuyst P, De Maertelaer V, et al. Comparison of computed density and microscopic morphometry in pulmonary emphysema. Am J Respir Crit Care Med 1996; 154: 187-192.

333 Muller NL, Staples CA, Miller RR, Abboud RT. “Density mask". An objective method to quantitate emphysema using computed tomography. Chest 1988; 94: 782-787.

334 Dowson LJ, Guest PJ, Hill SL, Holder RL, Stockley RA. High-resolution computed tomography scanning in $\alpha_{1^{-}}$ antitrypsin deficiency: relationship to lung function and health status. Eur Respir J 2001; 17: 1097-1104.

335 Parr DG, Stoel BC, Stolk J, Stockley RA. Pattern of emphysema distribution in $\alpha_{1}$-antitrypsin deficiency influences lung function impairment. Am J Respir Crit Care Med 2004; 170: 1172-1178.

336 Kinsella M, Muller NL, Abboud RT, Morrison NJ, DyBuncio A. Quantitation of emphysema by computed tomography using a "density mask" program and correlation with pulmonary function tests. Chest 1990; 97: 315-321.

337 Park KJ, Bergin CJ, Clausen JL. Quantitation of emphysema with three-dimensional CT densitometry: comparison with two-dimensional analysis, visual emphysema scores, and pulmonary function test results. Radiology 1999; 211: 541-547.

338 Nakano Y, Wong JC, de Jong PA, et al. The prediction of small airway dimensions using computed tomography. Am J Respir Crit Care Med 2005; 171: 142-146.

339 King GG, Muller NL, Whittall KP, Xiang QS, Pare PD. An analysis algorithm for measuring airway lumen and wall areas from high-resolution computed tomographic data. Am J Respir Crit Care Med 2000; 161: 574-580.

340 Berger P, Perot V, Desbarats P, Tunon-de-Lara JM, Marthan R, Laurent F. Airway wall thickness in cigarette smokers: quantitative thin-section CT assessment. Radiology 2005; 235: 1055-1064.

341 Matsuoka S, Kurihara Y, Nakajima Y, Niimi H, Ashida H, Kaneoya K. Serial change in airway lumen and wall thickness at thin-section CT in asymptomatic subjects. Radiology 2005; 234: 595-603.

342 Brillet PY, Fetita CI, Beigelman-Aubry C, et al. Quantification of bronchial dimensions at MDCT using dedicated software. Eur Radiol 2007; 17: 1483-1489.

343 Kemerink GJ, Lamers RJ, Thelissen GR, Van Engelshoven JM. CT densitometry of the lungs: scanner performance. J Comput Assist Tomogr 1996; 20: 24-33.

344 Parr DG, Stoel BC, Stolk J, Nightingale PG, Stockley RA. Influence of calibration on densitometric studies of emphysema progression using computed tomography. Am J Respir Crit Care Med 2004; 170: 883-890.

345 Kemerink GJ, Lamers RJ, Thelissen GR, Van Engelshoven JM. The nonlinear partial volume effect and computed tomography densitometry of foam and lung. Med Phys 1995; 22: 1445-1450.

346 Van Dyk J, Keane TJ, Rider WD. Lung density as measured by computerized tomography: implications for radiotherapy. Int J Radiat Oncol Biol Phys 1982; 8: 1363-1372.

347 Wegener $\mathrm{OH}$, Koeppe $\mathrm{P}$, Oeser H. Measurement of lung density by computed tomography. J Comput Assist Tomogr 1978; 2: 263-273.

348 Kalender WA, Rienmuller R, Seissler W, Behr J, Welke M, Fichte H. Measurement of pulmonary parenchymal attenuation: use of spirometric gating with quantitative CT. Radiology 1990; 175: 265-268.

349 Gierada DS, Yusen RD, Pilgram TK, et al. Repeatability of quantitative CT indexes of emphysema in patients evaluated for lung volume reduction surgery. Radiology 2001; 220: 448-454.

350 Hoffman EA, McLennan G. Assessment of the pulmonary structure-function relationship and clinical outcomes measures: quantitative volumetric CT of the lung. Acad Radiol 1997; 4: 758-776.

351 Shaker SB, Dirksen A, Laursen L, Skovgaard LT, Holstein-Rathlou NH. Volume adjustment of lung density by computed tomography scans in patients with emphysema. Acta Radiol 2004; 45: 417-423.

352 Stolk J, Dirksen A, van der Lugt AA, et al. Repeatability of lung density measurements with low-dose computed tomography in subjects with $\alpha_{1}$-antitrypsin deficiencyassociated emphysema. Invest Radiol 2001; 36: 648-651.

353 Lamers RJ, Kemerink GJ, Drent M, Van Engelshoven JM. Reproducibility of spirometrically controlled CT lung densitometry in a clinical setting. Eur Respir J 1998; 11 942-945.

354 Moroni C, Mascalchi M, Camiciottoli G, et al. Comparison of spirometric-gated and -ungated HRCT in COPD. J Comput Assist Tomogr 2003; 27: 375-379.

355 Newell JD, Hogg JC, Snider GL. Report of a workshop: quantitative computed tomography scanning in longitudinal studies of emphysema. Eur Respir J 2004; 23: 769-775.

356 Robinson PJ, Kreel L. Pulmonary tissue attenuation with computed tomography: comparison of inspiration and expiration scans. J Comput Assist Tomogr 1979; 3: 740-748.

357 Siegelman S. Pulmonary System: Practical Approaches to Pulmonary Diagnosis. New York, Grune and Stratton, 1979.

358 Camiciottoli G, Bartolucci M, Maluccio NM, et al. Spirometrically gated high-resolution CT findings in COPD: lung attenuation versus lung function and dyspnea severity. Chest 2006; 129: 558-564.

359 Levi C, Gray JE, McCullough EC, Hattery RR. The unreliability of CT numbers as absolute values. AJR Am J Roentgenol 1982; 139: 443-447.

360 Kemerink GJ, Lamers RJ, Thelissen GR, Van Engelshoven JM. Scanner conformity in CT densitometry of the lungs. Radiology 1995; 197: 749-752.

361 Kemerink GJ, Kruize HH, Lamers RJ, Van Engelshoven JM. CT lung densitometry: dependence of 
CT number histograms on sample volume and consequences for scan protocol comparability. J Comput Assist Tomogr 1997; 21: 948-954.

362 McCullough EC. Specifying and evaluating the performance of computed tomography (CT) scanners. Med Phys 1980; 7: 291-296.

363 Kemerink GJ, Kruize HH, Lamers RJ, Van Engelshoven JM. Density resolution in quantitative computed tomography of foam and lung. Med Phys 1996; 23: 1697-1708.

364 Stoel BC, Bakker ME, Stolk J, et al. Comparison of the sensitivities of 5 different computed tomography scanners for the assessment of the progression of pulmonary emphysema: a phantom study. Invest Radiol 2004; 39: 1-7.

365 Silverwright G, Elliot P. Interactive region and volume growing for segmentation volumes in $\mathrm{MR}$ and CT images. Med Inform 1994; 19: 71-80.

366 Zagers R, Vrooman HA, Aarts NJ, et al. Quantitative analysis of computed tomography scans of the lungs for the diagnosis of pulmonary emphysema. A validation study of a semiautomated contour detection technique. Invest Radiol 1995; 30: 552-562.

367 Stoel BC, Vrooman HA, Stolk J, Reiber JH. Sources of error in lung densitometry with CT. Invest Radiol 1999; 34: 303-309.

368 U.S. Food and Drug Administration. Guidance for Industry-computerized systems used in clinical trials. www.fda.gov/ora/compliance_ref/bimo/ffinalcct.htm Date last updated: January 1, 1997. Date last accessed: November 30, 2007.

369 Turner P, Whimster WF. Volume of emphysema. Thorax 1981; 36: 932-937.

370 Mishima M, Itoh H, Sakai H, et al. Optimized scanning conditions of high resolution CT in the follow-up of pulmonary emphysema. J Comput Assist Tomogr 1999; 23: 380-384.

371 Beinert $\mathrm{T}$, Behr J, Mehnert F, Kohz P, Seemann M, Reiser M. [Quantitative computerized tomography of the lung - respiration controlled diagnosis of diffuse lung diseases.] Pneumologie 1995; 49: 678-683.

372 Stolk J, Ng WH, Bakker ME, et al. Correlation between annual change in health status and computer tomography derived lung density in subjects with $\alpha_{1}$-antitrypsin deficiency. Thorax 2003; 58: 1027-1030.

373 Coxson $\mathrm{HO}$, Rogers RM, Whittall KP, et al. A quantification of the lung surface area in emphysema using computed tomography. Am J Respir Crit Care Med 1999; 159: 851-856.

374 Lamers RJ, Thelissen GR, Kessels AG, Wouters EF, Van Engelshoven JM. Chronic obstructive pulmonary disease: evaluation with spirometrically controlled CT lung densitometry. Radiology 1994; 193: 109-113.

375 Uppaluri R, Hoffman EA, Sonka M, Hunninghake GW, McLennan G. Interstitial lung disease: A quantitative study using the adaptive multiple feature method. Am J Respir Crit Care Med 1999; 159: 519-525.

376 Rosenblum LJ, Mauceri RA, Wellenstein DE, et al. Density patterns in the normal lung as determined by computed tomography. Radiology 1980; 137: 409-416.

377 Wollmer P, Albrechtsson U, Brauer K, Eriksson L, Jonson B, Tylen U. Measurement of pulmonary density by means of X-ray computerized tomography. Relation to pulmonary mechanics in normal subjects. Chest 1986; 90: 387-391.

378 Gevenois PA, Scillia P, De Maertelaer V, Michils A, De Vuyst P, Yernault JC. The effects of age, sex, lung size, and hyperinflation on CT lung densitometry. AJR Am J Roentgenol 1996; 167: 1169-1173.

379 Gillooly M, Lamb D. Airspace size in lungs of lifelong non-smokers: effect of age and sex. Thorax 1993; 48: 39-43.

380 Long FR, Williams RS, Castile RG. Inspiratory and expiratory CT lung density in infants and young children. Pediatr Radiol 2005; 35: 677-683.

381 Kohz P, Stabler A, Beinert $\mathrm{T}$, et al. Reproducibility of quantitative, spirometrically controlled CT. Radiology 1995; 197: 539-542.

382 Shaker SB, Dirksen A, Laursen LC, et al. Short-term reproducibility of computed tomography-based lung density measurements in $\alpha_{1}$-antitrypsin deficiency and smokers with emphysema. Acta Radiol 2004; 45: 424-430.

383 European Communities, ed. Council Directive 97/43/ Euratom of 30 June 1997 on health protection of individuals against the dangers of ionizing radiation in relation to medical exposure. Official Journal of the European Communities N. L190, 9.7.1997; 22.

384 van der Bruggen-Bogaarts BA, Broerse JJ, Lammers JW, van Waes PF, Geleijns J. Radiation exposure in standard and high-resolution chest CT scans. Chest 1995; 107: 113-115.

385 Radiological Protection in biomedical Research. A report of Committee 3 adopted by the International Commission of Radiological Protection. Oxford, Pergamon Press, 1993.

386 United Nations Scientific Committee on the effects of Atomic Radiation (UNSCEAR). Report to the General Assembly, with Scientific Annexes. New York, United Nations Publications, 1994.

387 ICRP. International Commission on Radiological Protection. Oxford, Pergamon Press, 1990.

388 Feinendegen LE, Bond VP, Sondhaus CA, Altman KI. Cellular signal adaptation with damage control at low doses versus the predominance of DNA damage at high doses. C R Acad Sci III 1999; 322: 245-251.

389 Cameron JR. A radiation unit for the public. Physics and Society News 1991; 20: 2.

390 Mayo JR, Aldrich J, Muller NL. Radiation exposure at chest CT: a statement of the Fleischner Society. Radiology 2003; 228: 15-21.

391 Lee KS, Primack SL, Staples CA, Mayo JR, Aldrich JE, Muller NL. Chronic infiltrative lung disease: comparison of diagnostic accuracies of radiography and low- and conventional-dose thin-section CT. Radiology 1994; 191: 669-673.

392 Skeletal muscle dysfunction in chronic obstructive pulmonary disease. A statement of the American Thoracic Society and European Respiratory Society. Am J Respir Crit Care Med 1999; 159: S1-S40.

393 Schols AM. Nutrition in chronic obstructive pulmonary disease. Curr Opin Pulm Med 2000; 6: 110-115.

394 Schols AM, Wouters EF, Soeters PB, Westerterp KR. Body composition by bioelectrical-impedance analysis compared with deuterium dilution and skinfold anthropometry in patients with chronic obstructive pulmonary disease. Am J Clin Nutr 1991; 53: 421-424. 
395 Baarends EM, Schols AM, Marken Lichtenbelt WD, Wouters EF. Analysis of body water compartments in relation to tissue depletion in clinically stable patients with chronic obstructive pulmonary disease. Am J Clin Nutr 1997; 65: 88-94.

396 Pichard C, Kyle UG, Janssens JP, et al. Body composition by X-ray absorptiometry and bioelectrical impedance in chronic respiratory insufficiency patients. Nutrition 1997; 13: 952-958.

397 Engelen MP, Schols AM, Does JD, Wouters EF. Skeletal muscle weakness is associated with wasting of extremity fat-free mass but not with airflow obstruction in patients with chronic obstructive pulmonary disease. Am J Clin Nutr 2000; 71: 733-738.

398 Pichard C, Kyle UG, Bracco D, Slosman DO, Morabia A, Schutz Y. Reference values of fat-free and fat masses by bioelectrical impedance analysis in 3393 healthy subjects. Nutrition 2000; 16: 245-254.

399 Steiner MC, Barton RL, Singh SJ, Morgan MD. Bedside methods versus dual energy X-ray absorptiometry for body composition measurement in COPD. Eur Respir J 2002; 19: 626-631.

400 Kyle UG, Piccoli A, Pichard C. Body composition measurements: interpretation finally made easy for clinical use. Curr Opin Clin Nutr Metab Care 2003; 6: 387-393.

401 Kyle UG, Genton L, Slosman DO, Pichard C. Fat-free and fat mass percentiles in 5225 healthy subjects aged 15 to 98 years. Nutrition 2001; 17: 534-541.

402 Schols AM, Dingemans AMC, Soeters PB, Wouters EF. Within-day variation of bioelectrical resistance measurements in patients with chronic obstructive pulmonary disease. Clinical Nutrition 1990; 9: 266-271.

403 Romero-Corral A, Montori VM, Somers VK, et al. Association of bodyweight with total mortality and with cardiovascular events in coronary artery disease: a systematic review of cohort studies. Lancet 2006; 368: 666-678.

404 Mostert R, Goris A, Weling-Scheepers C, Wouters EF, Schols AM. Tissue depletion and health related quality of life in patients with chronic obstructive pulmonary disease. Respir Med 2000; 94: 859-867.

405 Schols AM, Broekhuizen R, Weling-Scheepers CA, Wouters EF. Body composition and mortality in chronic obstructive pulmonary disease. Am J Clin Nutr 2005; 82: 53-59.

406 Creutzberg EC, Wouters EF, Mostert R, WelingScheepers CA, Schols AM. Efficacy of nutritional supplementation therapy in depleted patients with chronic obstructive pulmonary disease. Nutrition 2003; 19: 120-127.

407 Schols AMWJ, Brug J. Efficacy of nutritional intervention in chronic obstructive pulmonary disease. In: Wouters EFM, Schols AMWJ, eds. Nutrition and Metabolism in Chronic Respiratory Disease. Eur Respir Mon 2003; 8: 142-152.

408 Schols AM, Soeters PB, Mostert R, Pluymers RJ, Wouters EF. Physiologic effects of nutritional support and anabolic steroids in patients with chronic obstructive pulmonary disease. A placebo-controlled randomized trial. Am J Respir Crit Care Med 1995; 152: 1268-1274.

409 Franssen FM, Broekhuizen R, Janssen PP, Wouters EF, Schols AM. Effects of whole-body exercise training on body composition and functional capacity in normalweight patients with COPD. Chest 2004; 125: 2021-2028.

410 Creutzberg EC, Wouters EF, Mostert R, Pluymers RJ, Schols AM. A role for anabolic steroids in the rehabilitation of patients with COPD? A double-blind, placebocontrolled, randomized trial. Chest 2003; 124: 1733-1742.

411 Vermeeren MAP, Schols AMWJ, Qaudvlieg FCM, Wouters EFM. The influence of an acute disease exacerbation on the metabolic profile of patients with chronic obstructive pulmonary disease. Clin Nutr 1994; 13: Suppl. $1,38-39$.

412 de Godoy, I, Donahoe M, Calhoun WJ, Mancino J, Rogers RM., Elevated TNF- $\alpha$ production by peripheral blood monocytes of weight-losing COPD patients. Am J Respir Crit Care Med 1996; 153: 633-637.

413 Di Francia M, Barbier D, Mege JL, Orehek J. Tumor necrosis factor- $\alpha$ levels and weight loss in chronic obstructive pulmonary disease. Am J Respir Crit Care Med 1994; 150: 1453-1455.

414 Schols AM, Buurman WA, Staal van den Brekel AJ, Dentener MA, Wouters EF. Evidence for a relation between metabolic derangements and increased levels of inflammatory mediators in a subgroup of patients with chronic obstructive pulmonary disease. Thorax 1996; 51: 819-824.

415 Bolton CE, Ionescu AA, Shiels KM, et al. Associated loss of fat-free mass and bone mineral density in chronic obstructive pulmonary disease. Am J Respir Crit Care Med 2004; 170: 1286-1293.

416 Bernard S, Leblanc P, Whittom F, et al. Peripheral muscle weakness in patients with chronic obstructive pulmonary disease. Am J Respir Crit Care Med 1998; 158: 629-634.

417 Gosker HR, Lencer NH, Franssen FM, van der Vusse GJ, Wouters EF, Schols AM. Striking similarities in systemic factors contributing to decreased exercise capacity in patients with severe chronic heart failure or COPD. Chest 2003; 123: 1416-1424.

418 Gosselink R, Troosters T, Decramer M. Peripheral muscle weakness contributes to exercise limitation in COPD. Am J Respir Crit Care Med 1996; 153: 976-980.

419 Decramer M, Gosselink R, Troosters T, Verschueren M, Evers G. Muscle weakness is related to utilization of health care resources in COPD patients. Eur Respir J 1997; 10: 417-423.

420 Decramer $M$, de Bock V, Dom R. Functional and histologic picture of steroid-induced myopathy in chronic obstructive pulmonary disease. Am J Respir Crit Care Med 1996; 153: 1958-1964.

421 Spruit MA, Gosselink R, Troosters T, De Paepe K, Decramer $M$. Resistance versus endurance training in patients with COPD and peripheral muscle weakness. Eur Respir J 2002; 19: 1072-1078.

422 Franssen FM, Broekhuizen R, Janssen PP, Wouters EF, Schols AM. Limb muscle dysfunction in COPD: effects of muscle wasting and exercise training. Med Sci Sports Exerc 2005; 37: 2-9.

423 Wouters EF. Management of severe COPD. Lancet 2004; 364: 883-895.

424 Van Vliet M, Spruit MA, Verleden G, et al. Hypogonadism, quadriceps weakness, and exercise intolerance in chronic obstructive pulmonary disease. Am J Respir Crit Care Med 2005; 172: 1105-1111. 
425 Casaburi R, Bhasin S, Cosentino L, et al. Effects of testosterone and resistance training in men with chronic obstructive pulmonary disease. Am J Respir Crit Care Med 2004; 170: 870-878.

426 Urban RJ, Bodenburg YH, Gilkison C, et al. Testosterone administration to elderly men increases skeletal muscle strength and protein synthesis. Am J Physiol 1995; 269: E820-E826.

427 Arora NS, Rochester DF. Respiratory muscle strength and maximal voluntary ventilation in undernourished patients. Am Rev Respir Dis 1982; 126: 5-8.

428 Schunemann HJ, Puhan M, Goldstein R, Jaeschke R, Guyatt G. Measurement properties and interpretability of the chronic respiratory disease questionnaire (CRQ). J COPD 2005; 2: 81-89.

429 Schunemann HJ, Guyatt GH. Commentary - goodbye M(C)ID! Hello MID, where do you come from? Health Serv Res 2005; 40: 593-597.

430 Jaeschke R, Guyatt GH. How to develop and validate a new quality of life instrument. In: Spilker B, ed. Quality of Life Assessments in Clinical Trials. New York, Raven, 1990; pp. 47-57.

431 Norman GR, Sloan JA, Wyrwich KW. Interpretation of changes in health-related quality of life: the remarkable universality of half a standard deviation. Med Care 2003; 41: 582-592.

432 Cohen J. Statistical Power Analysis for the Behavioural Sciences. London, Academic Press, 1969.

433 Cazzola M. Application of number needed to treat (NNT) as a measure of treatment effect in respiratory medicine. Treat Respir Med 2006; 5: 79-84.

434 Leidy NK, Wyrwich KW. Bridging the gap: using triangulation methodology to estimate minimal clinically important differences (MCIDs). J COPD 2005; 2: 157-165.

435 Jones PW. St. George's Respiratory Questionnaire: MCID. J COPD 2005; 2: 75-79.

436 Mahler DA, Witek TJ Jr. The MCID of the transition dyspnea index is a total score of one unit. J COPD 2005; 2: 99-103.

437 Ries AL. Minimally clinically important difference for the UCSD shortness of breath questionnaire, Borg Scale, and Visual Analog Scale. J COPD 2005; 2: 105-110.

438 Sutherland ER, Make BJ. Maximum exercise as an outcome in COPD: minimal clinically important difference. J COPD 2005; 2: 137-141.

439 Wise RA, Brown CD. Minimal clinically important differences in the six-minute walk test and the incremental shuttle walking test. J COPD 2005; 2: 125-129.

440 Kaplan RM. The minimally clinically important differences in generic utility-based measures. J COPD 2005; 2: 91-97.

441 Wouters EF. Local and systemic inflammation in chronic obstructive pulmonary disease. Proc Am Thorac Soc 2005; 2: 26-33.

442 Calverley PMA. Minimal clinically important difference exacerbations of COPD. J COPD 2005; 2: 143-148.

443 Make B, Casaburi R, Leidy NK. Interpreting results from clinical trials: understanding minimal clinically important differences in COPD outcomes. COPD 2005; 2: 1-5.

444 Jones PW. Interpreting thresholds for a clinically significant change in health status in asthma and COPD. Eur Respir J 2002; 19: 398-404.
445 Jones PW, Quirk FH, Baveystock CM. The St George's Respiratory Questionnaire. Respir Med 1991; 85: Suppl. B, 25-31.

446 Jones PW, Bosh TK. Quality of life changes in COPD patients treated with salmeterol. Am J Respir Crit Care Med 1997; 155: 1283-1289.

447 Schunemann HJ, Griffith L, Jaeschke R, et al. A comparison of the original chronic respiratory questionnaire with a standardized version. Chest 2003; 124: 1421-1429.

448 van den Boom G, Rutten-van Mölken MP, Molema J, Tirimanna PR, van Weel C, van Schayck CP. The cost effectiveness of early treatment with fluticasone propionate 250 microg twice a day in subjects with obstructive airway disease. Results of the DIMCA program. Am J Respir Crit Care Med 2001; 164: 2057-2066.

449 Harper R, Brazier JE, Waterhouse JC, Walters SJ, Jones NM, Howard P. Comparison of outcome measures for patients with chronic obstructive pulmonary disease (COPD) in an outpatient setting. Thorax 1997; 52: 879-887.

450 Larson JL, Covey MK, Wirtz SE, et al. Cycle ergometer and inspiratory muscle training in chronic obstructive pulmonary disease. Am J Respir Crit Care Med 1999; 160: 500-507.

451 Lacasse Y, Brosseau L, Milne S, et al. Pulmonary rehabilitation for chronic obstructive pulmonary disease. Cochrane Database Syst Rev 2002; 3: CD003793.

452 Wyrwich KW, Fihn SD, Tierney WM, Kroenke K, Babu AN, Wolinsky FD. Clinically important changes in health-related quality of life for patients with chronic obstructive pulmonary disease: an expert consensus panel report. J Gen Intern Med 2003; 18: 196-202.

453 Jaeschke R, Singer J, Guyatt GH. Measurement of health status. Ascertaining the minimal clinically important difference. Control Clin Trials 1989; 10: 407-415.

454 Redelmeier DA, Guyatt GH, Goldstein RS. Assessing the minimal important difference in symptoms: a comparison of two techniques. J Clin Epidemiol 1996; 49: 1215-1219.

455 Witek TJ Jr, Mahler DA. Minimal important difference of the transition dyspnoea index in a multinational clinical trial. Eur Respir J 2003; 21: 267-272.

456 Guyatt GH, Pugsley SO, Sullivan MJ, et al. Effect of encouragement on walking test performance. Thorax 1984; 39: 818-822.

457 Leger LA, Lambert J. A maximal multistage 20-m shuttle run test to predict $V^{\prime} \mathrm{O}_{2}$ max. Eur J Appl Physiol Occup Physiol 1982; 49: 1-12.

458 ATS statement: guidelines for the six-minute walk test. Am J Respir Crit Care Med 2002; 166: 111-117.

459 Bernstein ML, Despars JA, Singh NP, Avalos K, Stansbury DW, Light RW. Reanalysis of the 12-minute walk in patients with chronic obstructive pulmonary disease. Chest 1994; 105: 163-167.

460 Wijkstra PJ, TenVergert EM, van der Mark TW, et al. Relation of lung function, maximal inspiratory pressure, dyspnoea, and quality of life with exercise capacity in patients with chronic obstructive pulmonary disease. Thorax 1994; 49: 468-472.

461 Guyatt GH, Thompson PJ, Berman LB, et al. How should we measure function in patients with chronic heart and lung disease? J Chronic Dis 1985; 38: 517-524. 
462 Redelmeier DA, Bayoumi AM, Goldstein RS, Guyatt GH. Interpreting small differences in functional status: the six minute walk test in chronic lung disease patients. Am J Respir Crit Care Med 1997; 155: 1278-1282.

463 Emtner M, Porszasz J, Burns M, Somfay A, Casaburi R. Benefits of supplemental oxygen in exercise training in nonhypoxemic chronic obstructive pulmonary disease patients. Am J Respir Crit Care Med 2003; 168: 1034-1042.

464 Casaburi R. Factors determining constant work rate exercise tolerance in COPD and their role in dictating the minimal clinically important difference in response to interventions. J COPD 2005; 2: 131-136.

465 Cox NJ, Hendriks JC, Binkhorst RA, Folgering HT, van Herwaarden CL. Reproducibility of incremental maximal cycle ergometer tests in patients with mild to moderate obstructive lung diseases. Lung 1989; 167: 129-133.

466 Donohue JF. Minimal clinically important differences in COPD lung function. J COPD 2005; 2: 111-124.

467 Schermer TR, Jacobs JE, Chavannes NH, et al. Validity of spirometric testing in a general practice population of patients with chronic obstructive pulmonary disease (COPD). Thorax 2003; 58: 861-866.

468 Enright PL, Beck KC, Sherrill DL. Repeatability of spirometry in 18,000 adult patients. Am J Respir Crit Care Med 2004; 169: 235-238.

469 Wise RA, Connett J, Kurnow K, et al. Selection of spirometric measurements in a clinical trial: the Lung Health Study. Am J Respir Crit Care Med 1995; 151: 675-681.

470 van Noord JA, Aumann JL, Janssens E, et al. Comparison of tiotropium once daily, formoterol twice daily and both combined once daily in patients with COPD. Eur Respir J 2005; 26: 214-222.

471 Redelmeier DA, Goldstein RS, Min ST, Hyland RH. Spirometry and dyspnea in patients with COPD. When small differences mean little. Chest 1996; 109: 1163-1168.

472 Franciosi LG, Page CP, Celli BR, et al. Markers of exacerbation severity in chronic obstructive pulmonary disease. Respir Res 2006; 7: 74.

473 Barnes PJ, Chowdhury B, Kharitonov SA, et al. Pulmonary biomarkers in chronic obstructive pulmonary disease. Am J Respir Crit Care Med 2006; 174: 6-14.

474 O'Shaughnessy TC, Ansari TW, Barnes NC, Jeffery PK. Inflammation in bronchial biopsies of subjects with chronic bronchitis: inverse relationship of CD8+ T lymphocytes with FEV1. Am J Respir Crit Care Med 1997; 155: 852-857.

475 Saetta M, Turato G, Maestrelli P, Mapp CE, Fabbri LM. Cellular and structural bases of chronic obstructive pulmonary disease. Am J Respir Crit Care Med 2001; 163: 1304-1309.

476 Saetta M, Di Stefano A, Maestrelli P, et al. Activated Tlymphocytes and macrophages in bronchial mucosa of subjects with chronic bronchitis. Am Rev Respir Dis 1993; 147: 301-306.

477 Panzner P, Lafitte JJ, Tsicopoulos A, Hamid Q, Tulic MK. Marked up-regulation of $\mathrm{T}$ lymphocytes and expression of interleukin-9 in bronchial biopsies from patients with chronic bronchitis with obstruction. Chest 2003; 124: 1909-1915.

478 Grumelli S, Corry DB, Song LZ, et al. An immune basis for lung parenchymal destruction in chronic obstructive pulmonary disease and emphysema. PLoS Med 2004; 1: e8.
479 Saetta M, Mariani M, Panina-Bordignon $P$, et al. Increased expression of the chemokine receptor CXCR3 and its ligand CXCL10 in peripheral airways of smokers with chronic obstructive pulmonary disease. Am J Respir Crit Care Med 2002; 165: 1404-1409.

480 Di Stefano A, Capelli A, Lusuardi M, et al. Severity of airflow limitation is associated with severity of airway inflammation in smokers. Am J Respir Crit Care Med 1998; 158: 1277-1285.

481 Saetta M, Di Stefano A, Maestrelli P, et al. Airway eosinophilia in chronic bronchitis during exacerbations. Am J Respir Crit Care Med 1994; 150: 1646-1652.

482 Zhu J, Qiu YS, Majumdar S, et al. Exacerbations of bronchitis: bronchial eosinophilia and gene expression for interleukin-4, interleukin-5, and eosinophil chemoattractants. Am J Respir Crit Care Med 2001; 164: 109-116.

483 Qiu Y, Zhu J, Bandi V, et al. Biopsy neutrophilia, neutrophil chemokine and receptor gene expression in severe exacerbations of chronic obstructive pulmonary disease. Am J Respir Crit Care Med 2003; 168: 968-975.

484 Hattotuwa KL, Gizycki MJ, Ansari TW, Jeffery PK, Barnes NC. The effects of inhaled fluticasone on airway inflammation in chronic obstructive pulmonary disease: a double-blind, placebo-controlled biopsy study. Am J Respir Crit Care Med 2002; 165: 1592-1596.

485 Verhoeven GT, Hegmans JP, Mulder PG, Bogaard JM, Hoogsteden HC, Prins JB. Effects of fluticasone propionate in COPD patients with bronchial hyperresponsiveness. Thorax 2002; 57: 694-700.

486 Gamble E, Grootendorst DC, Brightling CE, et al. Antiinflammatory effects of the phosphodiesterase-4 inhibitor cilomilast (Ariflo) in chronic obstructive pulmonary disease. Am J Respir Crit Care Med 2003; 168: 976-982.

487 Barnes NC, Qiu Y, Pavord I, et al. Salmeterol/fluticasone propionate (SFC): anti-inflammatory effects in COPD. Proc Am Thorac Soc 2005; 2: A543.

488 Jeffery $P$, Holgate $S$, Wenzel S. Methods for the assessment of endobronchial biopsies in clinical research: application to studies of pathogenesis and the effects of treatment. Am J Respir Crit Care Med 2003; 168: S1-S17.

489 Fuke S, Betsuyaku T, Nasuhara Y, Morikawa T, Katoh H, Nishimura M. Chemokines in bronchiolar epithelium in the development of chronic obstructive pulmonary disease. Am J Respir Cell Mol Biol 2004; 31: 405-412.

490 Hattotuwa K, Gamble EA, O'Shaughnessy T, Jeffery PK, Barnes NC. Safety of bronchoscopy, biopsy, and BAL in research patients with COPD. Chest 2002; 122: 1909-1912.

491 Löfdahl JM, Cederlund K, Nathell L, Eklund A, Skold CM. Bronchoalveolar lavage in COPD: fluid recovery correlates with the degree of emphysema. Eur Respir J 2005; 25: 275-281.

492 Culpitt SV, Rogers DF, Shah P, et al. Impaired inhibition by dexamethasone of cytokine release by alveolar macrophages from patients with chronic obstructive pulmonary disease. Am J Respir Crit Care Med 2003; 167: 24-31.

493 Russell RE, Culpitt SV, DeMatos C, et al. Release and activity of matrix metalloproteinase- 9 and tissue inhibitor of metalloproteinase-1 by alveolar macrophages from patients with chronic obstructive pulmonary disease. Am J Respir Cell Mol Biol 2002; 26: 602-609. 
494 Kalenderian R, Raju L, Roth W, Schwartz LB, Gruber B, Janoff A. Elevated histamine and tryptase levels in smokers' bronchoalveolar lavage fluid. Do lung mast cells contribute to smokers' emphysema? Chest 1988; 94: 119-123.

495 Pesci A, Balbi B, Majori M, et al. Inflammatory cells and mediators in bronchial lavage of patients with chronic obstructive pulmonary disease. Eur Respir J 1998; 12: 380-386.

496 Willemse BW, Postma DS, Timens W, ten Hacken NH. The impact of smoking cessation on respiratory symptoms, lung function, airway hyperresponsiveness and inflammation. Eur Respir J 2004; 23: 464-476.

497 Pesci A, Rossi GA, Bertorelli G, Aufiero A, Zanon P, Olivieri D. Mast cells in the airway lumen and bronchial mucosa of patients with chronic bronchitis. Am J Respir Crit Care Med 1994; 149: 1311-1316.

498 Ekberg-Jansson A, Andersson B, Bake B, et al. Neutrophilassociated activation markers in healthy smokers relates to a fall in DL,CO and to emphysematous changes on high resolution CT. Respir Med 2001; 95: 363-373.

499 Betsuyaku T, Nishimura M, Takeyabu K, Tanino M, Miyamoto K, Kawakami Y. Decline in FEV1 in community-based older volunteers with higher levels of neutrophil elastase in bronchoalveolar lavage fluid. Respiration 2000; 67: 261-267.

500 Skold CM, Hed J, Eklund A. Smoking cessation rapidly reduces cell recovery in bronchoalveolar lavage fluid, while alveolar macrophage fluorescence remains high. Chest 1992; 101: 989-995.

501 Andersson O, Cassel TN, Skold CM, Eklund A, Lund J, Nord M. Clara cell secretory protein. Levels in BAL fluid after smoking cessation. Chest 2000; 118: 180-182.

502 Pizzichini MM, Popov TA, Efthimiadis A, et al. Spontaneous and induced sputum to measure indices of airway inflammation in asthma. Am J Respir Crit Care Med 1996; 154: 866-869.

503 Tsoumakidou M, Tzanakis N, Siafakas NM. Induced sputum in the investigation of airway inflammation of COPD. Respir Med 2003; 97: 863-871.

504 Bhowmik A, Seemungal TA, Sapsford RJ, Devalia JL, Wedzicha JA. Comparison of spontaneous and induced sputum for investigation of airway inflammation in chronic obstructive pulmonary disease. Thorax 1998; 53: 953-956.

505 Taube C, Holz O, Mucke M, Jorres RA, Magnussen H. Airway response to inhaled hypertonic saline in patients with moderate to severe chronic obstructive pulmonary disease. Am J Respir Crit Care Med 2001; 164: 1810-1815.

506 Rytila PH, Lindqvist AE, Laitinen LA. Safety of sputum induction in chronic obstructive pulmonary disease. Eur Respir J 2000; 15: 1116-1119.

507 Paggiaro PL, Chanez P, Holz O, et al. Sputum induction. Eur Respir J 2002; 20: Suppl. 37, 3s-8s.

508 Brightling CE, Monteiro W, Ward R, et al. Sputum eosinophilia and short-term response to prednisolone in chronic obstructive pulmonary disease: a randomised controlled trial. Lancet 2000; 356: 1480-1485.

509 Brightling CE, McKenna S, Hargadon B, et al. Sputum eosinophilia and the short term response to inhaled mometasone in chronic obstructive pulmonary disease. Thorax 2005; 60: 193-198.
510 Tzanakis N, Chrysofakis G, Tsoumakidou M, et al. Induced sputum CD8+ T-lymphocyte subpopulations in chronic obstructive pulmonary disease. Respir Med 2004; 98: 57-65.

511 Keatings VM, Collins PD, Scott DM, Barnes PJ. Differences in interleukin-8 and tumor necrosis factoralpha in induced sputum from patients with chronic obstructive pulmonary disease or asthma. Am J Respir Crit Care Med 1996; 153: 530-534.

512 Keatings VM, Jatakanon A, Worsdell YM, Barnes PJ. Effects of inhaled and oral glucocorticoids on inflammatory indices in asthma and COPD. Am J Respir Crit Care Med 1997; 155: 542-548.

513 Culpitt SV, Maziak W, Loukidis S, Nightingale JA, Matthews JL, Barnes PJ. Effect of high dose inhaled steroid on cells, cytokines, and proteases in induced sputum in chronic obstructive pulmonary disease. Am J Respir Crit Care Med 1999; 160: 1635-1639.

514 Loppow D, Schleiss MB, Kanniess F, Taube C, Jorres RA, Magnussen $\mathrm{H}$. In patients with chronic bronchitis a four week trial with inhaled steroids does not attenuate airway inflammation. Respir Med 2001; 95: 115-121.

515 Culpitt SV, De Matos C, Russell RE, Donnelly LE, Rogers DF, Barnes PJ. Effect of theophylline on induced sputum inflammatory indices and neutrophil chemotaxis in chronic obstructive pulmonary disease. Am J Respir Crit Care Med 2002; 165: 1371-1376.

516 Beeh KM, Beier J, Kornmann O, Mander A, Buhl R. Longterm repeatability of induced sputum cells and inflammatory markers in stable, moderately severe COPD. Chest 2003; 123: 778-783.

517 Yamamoto C, Yoneda T, Yoshikawa M, et al. Airway inflammation in COPD assessed by sputum levels of interleukin-8. Chest 1997; 112: 505-510.

518 Aaron SD, Angel JB, Lunau M, et al. Granulocyte inflammatory markers and airway infection during acute exacerbation of chronic obstructive pulmonary disease. Am J Respir Crit Care Med 2001; 163: 349-355.

519 Culpitt S, Maziak W, Loukides S, Keller A, Barnes PJ. Effect of theophylline on induced sputum inflammatory indices in COPD patients. Am J Respir Crit Care Med 1997; 157: A797.

520 Culpitt SV, De Matos C, Russell RE, Donnelly LE, Rogers DF, Barnes PJ. Effect of theophylline on induced sputum inflammatory indices and neutrophil chemotaxis in chronic obstructive pulmonary disease. Am J Respir Crit Care Med 2002; 165: 1371-1376.

521 Hill AT, Bayley D, Stockley RA. The interrelationship of sputum inflammatory markers in patients with chronic bronchitis. Am J Respir Crit Care Med 1999; 160: 893-898.

522 Beeh KM, Beier J, Kornmann O, Buhl R. Sputum matrix metalloproteinase-9, tissue inhibitor of metalloprotinease-1, and their molar ratio in patients with chronic obstructive pulmonary disease, idiopathic pulmonary fibrosis and healthy subjects. Respir Med 2003; 97: 634-639.

523 Vernooy JH, Lindeman JH, Jacobs JA, Hanemaaijer R, Wouters EF. Increased activity of matrix metalloproteinase8 and matrix metalloproteinase- 9 in induced sputum from patients with COPD. Chest 2004; 126: 1802-1810. 
524 Culpitt SV, Rogers DF, Traves SL, Barnes PJ, Donnelly LE. Sputum matrix metalloproteases: comparison between chronic obstructive pulmonary disease and asthma. Respir Med 2005; 99: 703-710.

525 Holz O, Richter K, Jorres RA, Speckin P, Mucke M, Magnussen $\mathrm{H}$. Changes in sputum composition between two inductions performed on consecutive days. Thorax 1998; 53: 83-86.

526 Nightingale JA, Rogers DF, Barnes PJ. Effect of repeated sputum induction on cell counts in normal volunteers. Thorax 1998; 53: 87-90.

527 Kelly MM, Keatings V, Leigh R, et al. Analysis of fluidphase mediators. Eur Respir J 2002; 20: Suppl. 37, 24s-39s.

528 Erin EM, Barnes PJ, Hansel TT. Optimizing sputum methodology. Clin Exp Allergy 2002; 32: 653-657.

529 Kharitonov SA, Barnes PJ. Exhaled markers of pulmonary disease. Am J Respir Crit Care Med 2001; 163: 1693-1722.

530 Kharitonov SA, Barnes PJ. Biomarkers of some pulmonary diseases in exhaled breath. Biomarkers 2002; 7: 1-32.

531 Kharitonov S, Alving K, Barnes PJ. Exhaled and nasal nitric oxide measurements: recommendations. The European Respiratory Society Task Force. Eur Respir J 1997; 10: 1683-1693.

532 ATS/ERS Recommendations for Standardized Procedures for the Online and Offline Measurement of Exhaled Lower Respiratory Nitric Oxide and Nasal Nitric Oxide, 2005. Am J Respir Crit Care Med 2005; 171: 912-930.

533 Kharitonov SA, Gonio F, Kelly C, Meah S, Barnes PJ. Reproducibility of exhaled nitric oxide measurements in healthy and asthmatic adults and children. Eur Respir J 2003; 21: 433-438.

534 Maziak W, Loukides S, Culpitt S, Sullivan P, Kharitonov SA, Barnes PJ. Exhaled nitric oxide in chronic obstructive pulmonary disease. Am J Respir Crit Care Med 1998; 157: 998-1002.

535 Bhowmik A, Seemungal TA, Donaldson GC, Wedzicha JA. Effects of exacerbations and seasonality on exhaled nitric oxide in COPD. Eur Respir J 2005; 26: 1009-1015.

536 Rutgers SR, van der Mark TW, Coers W, et al. Markers of nitric oxide metabolism in sputum and exhaled air are not increased in chronic obstructive pulmonary disease. Thorax 1999; 54: 576-580.

537 Agusti AG, Villaverde JM, Togores B, Bosch M. Serial measurements of exhaled nitric oxide during exacerbations of chronic obstructive pulmonary disease. Eur Respir J 1999; 14: 523-528.

538 Montuschi P, Kharitonov SA, Barnes PJ. Exhaled carbon monoxide and nitric oxide in COPD. Chest 2001; 120: 496-501.

539 Kharitonov SA, Robbins RA, Yates D, Keatings V, Barnes PJ. Acute and chronic effects of cigarette smoking on exhaled nitric oxide. Am J Respir Crit Care Med 1995; 152: 609-612.

540 Brindicci C, Ito K, Resta O, Pride NB, Barnes PJ Kharitonov SA. Exhaled nitric oxide from lung periphery is increased in COPD. Eur Respir J 2005; 26: 52-59.

541 Maestrelli P, Paska C, Saetta M, et al. Decreased haem oxygenase-1 and increased inducible nitric oxide synthase in the lung of severe COPD patients. Eur Respir J 2003; 21: 971-976.
542 Paredi P, Kharitonov SA, Leak D, Ward S, Cramer D, Barnes PJ. Exhaled ethane, a marker of lipid peroxidation, is elevated in chronic obstructive pulmonary disease. Am J Respir Crit Care Med 2000; 162: 369-373.

543 Montuschi P, Barnes PJ. Analysis of exhaled breath condensate for monitoring airway inflammation. Trends Pharmacol Sci 2002; 23: 232-237.

544 Horvath I, Hunt J, Barnes PJ. Exhaled breath condensate: methodological recommendations and unresolved questions. Eur Respir J 2005; 26: 523-548.

545 Dekhuijzen PN, Aben KK, Dekker I, et al. Increased exhalation of hydrogen peroxide in patients with stable and unstable chronic obstructive pulmonary disease. Am J Respir Crit Care Med 1996; 154: 813-816.

546 Kostikas K, Papatheodorou G, Psathakis K, Panagou P, Loukides $S$. Oxidative stress in expired breath condensate of patients with COPD. Chest 2003; 124: 1373-1380.

547 Gerritsen WB, Asin J, Zanen P, van den Bosch JM, Haas FJ. Markers of inflammation and oxidative stress in exacerbated chronic obstructive pulmonary disease patients. Respir Med 2005; 99: 84-90.

548 Montuschi P, Collins JV, Ciabattoni G, et al. Exhaled 8isoprostane as an in vivo biomarker of lung oxidative stress in patients with COPD and healthy smokers. Am J Respir Crit Care Med 2000; 162: 1175-1177.

549 Carpagnano GE, Resta O, Foschino-Barbaro MP, et al. Exhaled interleukin-6 and 8-isoprostane in chronic obstructive pulmonary disease: effect of carbocysteine lysine salt monohydrate (SCMC-Lys). Eur J Pharmacol 2004; 505: 169-175.

550 Carpagnano GE, Kharitonov SA, Foschino-Barbaro MP, Resta O, Gramiccioni E, Barnes PJ. Supplementary oxygen in healthy subjects and those with COPD increases oxidative stress and airway inflammation. Thorax 2004; 59: 1016-1019.

551 Biernacki WA, Kharitonov SA, Barnes PJ. Increased leukotriene $\mathrm{B}_{4}$ and 8-isoprostane in exhaled breath condensate of patients with exacerbations of COPD. Thorax 2003; 58: 294-298.

552 Corradi M, Rubinstein I, Andreoli R, et al. Aldehydes in exhaled breath condensate of patients with chronic obstructive pulmonary disease. Am J Respir Crit Care Med 2003; 167: 1380-1386.

553 Corradi M, Montuschi P, Donnelly LE, Pesci A, Kharitonov SA, Barnes PJ. Increased nitrosothiols in exhaled breath condensate in inflammatory airway diseases. Am J Respir Crit Care Med 2001; 163: 854-858.

554 Kostikas K, Papatheodorou G, Ganas K, Psathakis K, Panagou P, Loukides S. pH in expired breath condensate of patients with inflammatory airway diseases. Am J Respir Crit Care Med 2002; 165: 1364-1370.

555 Borrill Z, Starkey C, Vestbo J, Singh D. Reproducibility of exhaled breath condensate $\mathrm{pH}$ in chronic obstructive pulmonary disease. Eur Respir J 2005; 25: 269-274.

556 Montuschi P, Barnes PJ. Exhaled leukotrienes and prostaglandins in asthma. J Allergy Clin Immunol 2002; 109: 615-620.

557 Kostikas K, Gaga M, Papatheodorou G, Karamanis T, Orphanidou D, Loukides S. Leukotriene $B_{4}$ in exhaled breath condensate and sputum supernatant in patients with COPD and asthma. Chest 2005; 127: 1553-1559. 
558 Bucchioni E, Kharitonov SA, Allegra L, Barnes PJ. High levels of interleukin- 6 in the exhaled breath condensate of patients with COPD. Respir Med 2003; 97: 1299-1302.

559 Effros RM, Su J, Casaburi R, Shaker R, Biller J, Dunning M. Utility of exhaled breath condensates in chronic obstructive pulmonary disease: a critical review. Curr Opin Pulm Med 2005; 11: 135-139.

560 Effros RM, Peterson B, Casaburi R, et al. Epithelial lining fluid solute concentrations in chronic obstructive lung disease patients and normal subjects. J Appl Physiol 2005; 99: 1286-1292.

561 Cap P, Chladek J, Pehal F, et al. Gas chromatography/ mass spectrometry analysis of exhaled leukotrienes in asthmatic patients. Thorax 2004; 59: 465-470.

562 Montuschi P, Ragazzoni E, Valente S, et al. Validation of leukotriene $\mathrm{B}_{4}$ measurements in exhaled breath condensate. Inflamm Res 2003; 52: 69-73.

563 Sin DD, Man SF. Skeletal muscle weakness, reduced exercise tolerance, and COPD: is systemic inflammation the missing link? Thorax 2006; 61: 1-3.

564 Gan WQ, Man SF, Sin DD. The interactions between cigarette smoking and reduced lung function on systemic inflammation. Chest 2005; 127: 558-564.

565 Man SF, Sin DD. Effects of corticosteroids on systemic inflammation in chronic obstructive pulmonary disease. Proc Am Thorac Soc 2005; 2: 78-82.

566 Gan WQ, Man SF, Senthilselvan A, Sin DD. Association between chronic obstructive pulmonary disease and systemic inflammation: a systematic review and a metaanalysis. Thorax 2004; 59: 574-580.

567 Sin DD, Lacy P, York E, Man SF. Effects of fluticasone on systemic markers of inflammation in chronic obstructive pulmonary disease. Am J Respir Crit Care Med 2004; 170: 760-765.

568 Spruit MA, Gosselink R, Troosters T, Kasran A, Van Vliet M, Decramer M. Low-grade systemic inflammation and the response to exercise training in patients with advanced COPD. Chest 2005; 128: 3183-3190.

569 Asero V, Mistretta A, Arcidiacono G, Polosa R. The puzzling relationship between cigarette smoking, reduced respiratory function, and systemic inflammation. Chest 2005; 128: 3772-3773.

570 Agusti AG. Systemic effects of chronic obstructive pulmonary disease. Proc Am Thorac Soc 2005; 2: 367-370.

571 Donaldson GC, Seemungal TA, Patel IS, et al. Airway and systemic inflammation and decline in lung function in patients with COPD. Chest 2005; 128: 1995-2004.

572 Hurst JR, Perera WR, Wilkinson TM, Donaldson GC, Wedzicha JA. Systemic and upper and lower airway inflammation at exacerbation of chronic obstructive pulmonary disease. Am J Respir Crit Care Med 2006; 173: 71-78.

573 Pinto-Plata VM, Mullerova H, Toso JF, et al. C-reactive protein in patients with COPD, control smokers and nonsmokers. Thorax 2006; 61: 23-28.

574 van Eeden SF, Yeung A, Quinlam K, Hogg JC. Systemic response to ambient particulate matter: relevance to chronic obstructive pulmonary disease. Proc Am Thorac Soc 2005; 2: 61-67.

575 Broekhuizen R, Wouters EF, Creutzberg EC, Schols AM. Raised CRP levels mark metabolic and functional impairment in advanced COPD. Thorax 2006; 61: 17-22.

576 van Helvoort HA, van de Pol MH, Heijdra YF, Dekhuijzen PN. Systemic inflammatory response to exhaustive exercise in patients with chronic obstructive pulmonary disease. Respir Med 2005; 99: 1555-1567.

577 Koechlin C, Couillard A, Cristol JP, et al. Does systemic inflammation trigger local exercise-induced oxidative stress in COPD? Eur Respir J 2004; 23: 538-544.

578 Oudijk E-JD, Lammers J-WJ, Koenderman L. Systemic inflammation in chronic obstructive pulmonary disease. Eur Respir J 2003; 22: Suppl. 46, 5s-13s.

579 Andreassen H, Vestbo J. Chronic obstructive pulmonary disease as a systemic disease: an epidemiological perspective. Eur Respir J 2003; 22: Suppl. 46, 2s-4s.

580 Vernooy JH, Kucukaycan M, Jacobs JA, et al. Local and systemic inflammation in patients with chronic obstructive pulmonary disease: soluble tumor necrosis factor receptors are increased in sputum. Am J Respir Crit Care Med 2002; 166: 1218-1224.

581 Vila S, Miravitlles M, Campos F, et al. [Importance of serum interleukin- 6 as a mediator of systemic inflammation in patients with $\alpha_{1}$-antitrypsin deficiency.] Arch Bronconeumol 2002; 38: 263-266.

582 Malo O, Sauleda J, Busquets X, Miralles C, Agusti AG, Noguera A. [Systemic inflammation during exacerbations of chronic obstructive pulmonary disease.] Arch Bronconeumol 2002; 38: 172-176.

583 Eid AA, Ionescu AA, Nixon LS, et al. Inflammatory response and body composition in chronic obstructive pulmonary disease. Am J Respir Crit Care Med 2001; 164: 1414-1418.

584 Nguyen LT, Bedu M, Caillaud D, et al. Increased resting energy expenditure is related to plasma TNF- $\alpha$ concentration in stable COPD patients. Clin Nutr 1999; 18: 269-274.

585 Barnes PJ, Hansel TT. Prospects for new drugs for chronic obstructive pulmonary disease. Lancet 2004; 364: 985-996. 